\title{
Ferrous-glutathione coupling mediates ferroptosis and frailty in Caenorhabditis elegans
}

Nicole L. Jenkins ${ }^{1}$, Simon A. James ${ }^{1,}$ Agus Salim ${ }^{2,3}$, Fransisca Sumardy ${ }^{1}$, Terence P. Speed ${ }^{3,4}$, Marcus Conrad $^{5}$, Des R. Richardson ${ }^{6}$, Ashley I. Bush ${ }^{1 *} \&$ Gawain McColl $^{1 *}$

${ }^{1}$ Melbourne Dementia Research Centre, Florey Institute of Neuroscience and Mental Health and University of Melbourne, Parkville, 3052 Victoria, Australia.

${ }^{2}$ Department of Mathematics and Statistics, La Trobe University, Bundoora 3083, Victoria, Australia.

${ }^{3}$ Bioinformatics Division, Walter and Eliza Hall Institute of Medical Research, Parkville 3052, Victoria, Australia.

${ }^{4}$ Department of Mathematics and Statistics, University of Melbourne, Parkville VIC 3010, Australia

${ }^{5}$ Helmholtz Zentrum München, Institute of Developmental Genetics, 85764 Neuherberg, Germany.

${ }^{6}$ Department of Pathology and Bosch Institute, University of Sydney, Sydney 2006, New South Wales, Australia

*Corresponding authors: ashley.bush@florey.edu.au; gawain.mccoll@florey.edu.au

All eukaryotes require iron. Replication, detoxification, and a cancer-protective form of regulated cell death termed ferroptosis ${ }^{1}$, all depend on iron metabolism. Ferrous iron accumulates over adult lifetime in the Caenorhabditis elegans model of ageing ${ }^{2}$. Here we show that glutathione depletion is coupled to ferrous iron elevation in these animals, and that both occur in late life to prime cells for ferroptosis. We demonstrate that blocking ferroptosis, either by inhibition of lipid peroxidation or by limiting iron retention, mitigates age-related cell death and markedly increases lifespan and healthspan in $C$. elegans. Temporal scaling of lifespan is not evident when ferroptosis is inhibited, consistent with this cell death process acting at specific life phases to induce organismal frailty, rather than contributing to a constant ageing rate. Because excess age-related iron elevation in somatic tissue, particularly in brain ${ }^{3-5}$, is thought to contribute to degenerative disease ${ }^{6,7}$, our data indicate that post-developmental interventions to limit ferroptosis may promote healthy ageing.

Ferroptosis is a regulated cell death program genetically and biochemically distinct from apoptosis, necrosis and autophagic cell death. It kills malignant cells but may also be inappropriately activated in ischemic injury and neurodegeneration ${ }^{8-11}$. This cell death mechanism is executed by (phospho)lipid hydroperoxides induced by either iron-dependent lipoxygenases, or by an iron-catalyzed spontaneous peroxyl radical-mediated chain reaction (autoxidation). Under homeostatic conditions the ferroptotic signal is terminated by glutathione peroxide-4 (GPx4), a phospholipid hydroperoxidase that needs glutathione as a cofactor. While the signaling that regulates ferroptosis has been studied in depth ${ }^{12-14}$, the role of iron load in this death signal is poorly resolved ${ }^{15}$.

Redox cycling between $\mathrm{Fe}^{2+}$ and $\mathrm{Fe}^{3+}$ can contribute to cellular stress. This is mitigated by a range of storage and chaperone pathways to ensure that the labile iron pool is kept to a minimum ${ }^{6}$. In Caenorhabditis elegans the emergence of labile ferrous iron with age correlates with genetic effects that accelerate ageing ${ }^{2,16}$ and could be a lifespan hazard ${ }^{17}$. Excess iron supply has been shown to shorten lifespan in C. elegans ${ }^{18,19}$, however variable results have been reported with iron chelation. The iron chelator deferiprone was reported not to impact $C$. elegans lifespan ${ }^{20}$, but this study was limited by 
indirect measures of iron load, use of only a single dose of deferiprone, and small sample size. In contrast, use of calcium-ethylenediaminetetraacetic acid (CaEDTA), a non-specific chelator that does not redoxsilence iron, caused a minor (undisclosed) increase in lifespan ${ }^{21}$. Whether selective targeting of ferrous iron burden can impact on ageing and lifespan is unknown.

We contemplated whether the developmental dependence on iron for reproduction and cellular biochemistry could represent an ancient and conserved liability in late life. The load of tissue iron increases needlessly in ageing nematodes, mammals and humans ${ }^{2-5}$. This must tax regulatory systems that prevent abnormal redox cycling of iron, such as the $\mathrm{Fe}^{2+}$-glutathione complexes considered the dominant form of iron in the cellular labile iron pool $^{22}$. We hypothesized that age-dependent elevation of labile iron, coupled with a reduction of glutathione levels conspire to lower the threshold for ferroptotic signaling, increasing the vulnerability of aged animals and implying that disruption to the ironglutathione axis is fundamental to natural ageing and death. To test this, we investigated the vulnerability to ferroptosis of ageing nematodes upon the natural loss of glutathione during lifespan. We examined the effects of inhibiting ferroptosis in C. elegans using two distinct treatments: a potent quenching agent for lipid peroxidation (autoxidation) ${ }^{23}$, as well as a small lipophilic iron chelator ${ }^{24}$ that prevents the initiation and amplification of lipid peroxide signals. Our analysis of these interventions provides mechanistic insight into the influence of the $\mathrm{Fe}^{2+}$-glutathione couple on the occurrence of natural ferroptosis across lifespan, at the cellular and organismal level.

\section{Results}

Glutathione depletion vulnerability. Glutathione is not only the dominant coordinating ligand for cytosolic ferrous iron ${ }^{22}$ but is also the substrate used by glutathione peroxidase-4 (GPX4) to clear the lipid peroxides that induce ferroptotic cell death ${ }^{25-27}$. Deletion of four C. elegans homologs of GPX4 decreases lifespan ${ }^{28}$, but whether ferroptosis is responsible is unknown. We tested whether acute depletion of glutathione can initiate ferroptosis in adult C. elegans using diethyl maleate (DEM), which conjugates glutathione ${ }^{29,30}$. We found that DEM induced death in 4-day old worms (at the end of their reproductive phase) in a dose- and time-dependent manner, with $\approx 50 \%$ lethality occurring after 24 -hour exposure to $10 \mathrm{mM}$ DEM (Figure 1A) associated with $\approx 50 \%$ depletion of glutathione (Figure 1B). We also found that total glutathione levels steadily decrease with normal ageing, approaching $\approx 50 \%$ on Day 10 of the levels on Day 1 (Figure 1C, Supplemental Table S1), and that C. elegans become disproportionately more vulnerable to DEM lethality as they enter the midlife stage (Figure 1D).

We tested whether lethality associated with glutathione depletion was caused by ferroptosis. We examined the treatment of $C$. elegans with the selective ferroptosis inhibitor, liproxstatin (Lip-1, 200 $\mu \mathrm{M})^{1}$. We also targeted the accumulation of late life iron ${ }^{2,16}$, that catalyses (phospho)lipid hydroperoxide propagation, using salicylaldehyde isonicotinoyl hydrazone ( $\mathrm{SIH}, 250 \mu \mathrm{M})$, a lipophilic acylhydrazone that scavenges intracellular iron and mobilizes it for extracellular clearance ${ }^{24}$. Importantly, unlike chelators such as CaEDTA, SIH binds iron in a redox-silent manner ${ }^{31}$. For both interventions, C. elegans were treated from early adulthood (late L4) onwards to eliminate any potential developmental effects.

DEM toxicity in 4-day old worms was rescued by both Lip-1 and SIH (Figure 1E, Supplemental Table S2), with more marked protection by SIH. This is consistent with ferroptosis contributing to the death mechanism. Therefore, the fall in glutathione with ageing (Figure 1C, Table S1) would be expected to interact synergistically with the concomitant rise in labile iron ${ }^{2,16}$ to increase the risk of ferroptosis. We found that this age-dependent rise in iron itself contributes to the fall in glutathione, since pretreatment of the worms with SIH from L4 prevented the age-dependent decrease in glutathione when assayed on Day 4 of adult life (Figure 1F). Furthermore, SIH mitigated the glutathione depletion induced by DEM in Day 4 animals (Figure 1F), demonstrating that cytosolic iron synergizes the depletion of 
glutathione initiated by DEM. While Lip-1 alleviated the lethality of DEM (Figure 1E), it did not prevent the fall in glutathione that was induced by ageing (as assayed on Day 4) or by DEM (Figure 1F). Thus, Lip-1 inhibition of ferroptosis in C. elegans occurs downstream of glutathione depletion, consistent with its effect in rescuing ferroptosis in cultured cells ${ }^{32}$.

Individual cell ferroptosis heralds organismal demise. A feature of ferroptosis is the propagation of cell death in a paracrine manner mediated by uncertain signals that might include the toxic lipid peroxidation end-products 4-hydroxynonenal (4-HNE) and malondialdehyde (MDA) ${ }^{33,34}$. Compared to strong oxidants like the hydroxyl radical, 4-HNE and MDA are relatively stable and able react with macromolecules, such as proteins distal to the site of origin. To determine whether individual cell death precedes organismal death in our model of ageing, we used propidium iodide to visualize dead cells in vivo after DEM treatment and during ageing. Propidium iodide is a fluorescent intercalating agent that binds to DNA, but cannot cross the membrane of live cells, making it possible to identify the nuclei of recently dead or dying cells, as shown in Figure 2A. Examination of aged cohorts, or young animals treated with DEM, indicated that cell death (particularly death of intestinal cells) preceded organismal death in both 4 day old (Figure 2B) and 8 day old (Figure 2C) adults, and was significantly attenuated by both Lip-1 or SIH. Thus, the animal dies cell by cell, rather than in a single event, and this progressive degeneration is likely to contribute to the frailty phenotype.

To estimate changes in lipid peroxidation, we assayed MDA via the thiobarbituric acid reactive substance assay. As expected, acute glutathione depletion by DEM exposure caused a marked increase in the relative amounts of MDA (Figure 2D). We also observed an aged-related increase in MDA, consistent with an age-related increase in ferroptotic signalling in $C$. elegans, that was ameliorated by both Lip-1 and SIH treatment (Figure 2E). Consistent with the MDA results, we also found a concomitant qualitative increase in 4-HNE protein adducts with age that was suppressed by both Lip-1 and SIH treatments (Figure 2F).

Changes in iron quantity, speciation and cytoplasmic fraction. Lowering cellular iron suppresses ferroptosis, but the peroxyl radical trapping ferroptosis inhibitors, such as Lip-1, are not expected to change iron levels. We examined the impact of SIH and Lip-1 interventions on iron levels over lifespan using synchrotron-based X-ray fluorescence microscopy ${ }^{2,35}$ to measure both iron concentration (presented as areal density, pg $\mu^{-2}$ ) and total (pg per worm) iron (Figure 3A; Supplemental Figure S1). Both total iron and areal density increased with age in control animals (Figure 3B \& C; Supplemental Tables S3\&S4), as expected ${ }^{2}$. SIH dramatically reduced the areal density of iron (and reduced variance) with ageing (Figure 3C; Supplemental Table S3\&S4), but Lip-1 did not alter iron density. Notably, by Day 8, animals treated with SIH contained total iron load on par with the untreated control group (Figure 3C; Supplemental Tables S5\&S6), as the lower areal density was offset by an increase in body size of SIH-treated worms compared to age matched controls. These results highlight how bulk measures of total iron or measurements by inference ${ }^{20}$ can be confounded by changes in the animal morphology when exploring ageing interventions.

We had previously determined age-related changes to the $C$. elegans iron-proteome, characterized on size exclusion chromatography by three major peaks: a high molecular weight peak (HMW, >1 MDa), ferritin, and a low MW peak (LMW, $600 \mathrm{Da}$ ) that may contain labile iron. With ageing, iron redistributes in C. elegans out of the ferritin peak (where it is sequestered in redox-silent storage reserves) and accumulates in the HMW and LMW peaks ${ }^{2}$. The chromatographic profile of aged C. elegans (10 days post adulthood) treated with SIH (Figure 3D) revealed decreased iron associated with the LMW peak (normalized peak area approximately 40\%). Ferritin-bound iron was also similarly decreased by SIH (normalized peak area approximately 50\%), but iron bound within HMW species was unaffected. The age-related changes in LMW iron are consistent with increased labile iron, which is withdrawn as the substrate for ferroptosis by SIH treatment. 
$\mathrm{Fe}^{2+}$ increase with ageing is normalized by liproxstatin and SIH. X-ray absorption near edge structure (XANES) spectroscopy, using fluorescence detection for visualization, directly assesses the in vivo coordination environments of metal ions in biological specimens ( $\varphi$ XANES) ${ }^{16}$. The centroid of the XANES pre-edge feature reflects the relative abundance of ferrous $\left[\mathrm{Fe}^{2+}\right]$ and ferric $\left[\mathrm{Fe}^{3+}\right]$ species $^{36}$. Since $\mathrm{Fe}^{2+}$ in the labile iron pool is the specific substrate for ferroptosis, and rises with ageing in $C$. elegans $^{2}$, we investigated the impact of our interventions using $\varphi \mathrm{XANES}^{16}$. This synchrotron-based spectroscopy allowed us to evaluate steady state iron speciation $\left(\mathrm{Fe}^{2+} / \mathrm{Fe}^{3+}\right)$ in a specific region (anterior intestinal, Figure 4A; Supplemental Figure S2) of intact, cryogenically-stabilized control, Lip-1 and $\mathrm{SIH}$-treated worms. We found that the age-related increase in the $\mathrm{Fe}^{2+}$ fraction was normalized to that of a young animal by both Lip-1 and SIH treatments (Figure 4B \& C; Supplemental Figure S3A \& B; Supplemental Table S7).

Higher levels of pro-ferroptotic $\mathrm{Fe}^{2+}$ might be compounded by a loss of glutathione. So, we also assessed changes in fractional $\mathrm{Fe}^{2+}$ induced by lethal glutathione depletion by DEM. $\varphi$ XANES of 4 day old wild type worms treated with DEM identified a marked increase in the $\mathrm{Fe}^{2+}$ fraction (Figure 4D; Supplemental Figure S3C \& D; Supplemental Table S7), revealing the upper limit for tolerable $\mathrm{Fe}^{2+}$ fraction being about 0.3 of the total iron (Figure 4D). These results help to contextualize the observed increase in $\mathrm{Fe}^{2+}$ during normal ageing also being about 0.3 of the total iron (Figure 4C), which was normalized to $\approx 0.2$ by Lip-1 or SIH intervention.

Lifespan effects of ferroptosis inhibition or blocking iron accumulation. Since $\mathrm{Fe}^{2+}$ accumulates with ageing and contributes to $C$. elegans frailty by executing cells before organismal death, we hypothesized that ferroptosis directly impacts on lifespan and may represent an underlying process that contributes to organismal ageing. We found that treatment of C. elegans with Lip-1 markedly extended lifespan [Figure 5A\&B; average $\sim 70 \%$ increase in median lifespan ( 8 independent replicates; $p<0.002$ ), Supplemental Table S8]. Dose response is shown in Supplemental Figures S4A \& B. An alternative ferroptosis inhibitor, ferrostatin ${ }^{1}$, was also examined, producing a significant but more modest median lifespan extension (Supplemental Figure S4C). Targeting the accumulation of late life iron using SIH also resulted in a marked increase in median lifespan [Figure 5A\&B; average $\sim 100 \%$ median increase (8 independent replicates; $p<0.0001$ ), Supplemental Table S8]. Dose response is shown in Supplemental Figure S4D. Exposing $C$. elegans to $250 \mu \mathrm{M}$ SIH as an iron complex $\left(\mathrm{Fe}(\mathrm{SIH})_{2} \mathrm{NO}_{3}\right)$ neutralized the benefits of SIH on lifespan (Supplemental Figure S4E), confirming that the rescue mechanism required SIH being free to ligate iron.

Lifespan increases are not due to temporal scaling. Lip-1 and SIH had distinct and unprecedented effects on ageing, as shown by the lifespan curves in Figure 5. Treatment with Lip-1 primarily altered late life survival, while SIH extended mid-life with a squaring of the survival curve. Interventions that increase lifespan in C. elegans are not uncommon, but Stroustrup et al. recently demonstrated the great majority of longevity interventions e.g. dietary and temperature alteration, oxidative stress, and genetic disruptions of the insulin/IGF-1 pathway (e.g. daf-2 and daf-16), heat shock factor $h s f-1$, or hypoxiainducible factor hif-1, each alter lifespan by temporal scaling - an apparent stretching or shrinking of time ${ }^{37}$. For an intervention to extend lifespan by temporal scaling it must alter, to the same extent throughout adult life, all physiological determinants of the risk of death. In effect temporal scaling arises when the risk of death is modulated by an intervention acting solely on the rate constant associated with a single stochastic process.

Combining the replicate data from 8 independent experiments (Figure 5B), we assessed whether Lip-1 and SIH treatment effects can be explained by the temporal scaling model of accelerated failure time (AFT). We found that the lifespan increases were not consistent with the temporal scaling model $(p<0.01$; Supplemental Tables S9-S14 and Figures S5-S10), so the interventions must target previously unrecognized ageing mechanisms. For SIH treatment, the risk of death (hazard) in early adulthood was 
greatly reduced compared to control populations but rose precipitously in late life (Figure 5C). Lip-1 markedly reduced the rate of mortality in the post-reproductive period (late-life) with early life mortality closer to that seen in untreated populations. These findings are consistent with ferroptotic cell death limiting lifespan in late life rather than being a global regulator (e.g. insulin/IGF-1 pathway) of ageing. This raises the possibility of targeted intervention with minimal or no metabolic cost.

The sample size in our experiment is much smaller than the lifespan machine experiment undertaken by Stroustrup et al. ${ }^{37}$ yet the data against temporal rescaling were significant. To minimize the likelihood that our findings are due to either intrinsic bias in our experiment or inflation of effect size (the Winner's curse phenomenon) we also examined the effect of temperature on lifespan intervention. Stroustrup et $a l .{ }^{37}$ reported that changing temperature results in simple temporal rescaling of lifespans; our data corroborated this result, and showed that SIH still extended lifespan by a similar dimension at both 20 ${ }^{\circ} \mathrm{C}$ and $25^{\circ} \mathrm{C}$ (Supplemental Tables S15-S17, Supplemental Figure S11).

Our results indicate that while iron accumulation may impact many processes that influence ageing rate, ferroptosis inhibition predominantly reduces frailty rather than slows a global rate of ageing. Notably, Stroustrup et al. identified only two other instances among the many lifespan interventions tested in $C$. elegans that modulated lifespan outside a temporal scaling model, namely altered feeding behaviour (eat-2 mutants) and mitochondrial dysfunction (nuo-6 mutants) ${ }^{37}$. Both these mutants express marked developmental variability and reduced fitness.

Preventing ferroptosis improves fitness and healthspan. Interventions that increase lifespan in $C$. elegans often do so at the detriment of fitness and healthspan. Adult body size can inform on fitness; reduced size may reflect a trade-off between longevity and fitness, as typically seen under dietary restriction where the cost of increased longevity can be lowered size, fertility and movement ${ }^{38}$. Distinctly, SIH-treated animals grew substantially larger. Following one day of treatment all animals were of similar body length (Figure 6A \& B). After 4 days and 8 days of intervention, adult SIH-treated animals were significantly longer compared to similarly aged controls (e.g. control $1440 \pm 123 \mu \mathrm{m}$ versus SIH $1696 \pm$ $64 \mu \mathrm{m}$, means $\pm \mathrm{SD}$ on Day 8, $p<0.001)$. In addition, SIH induced an increase in body volume between Days 1 and 4, but not thereafter (Figure 6C). SIH-treated worms grew to greater volume than both control and Lip-1 treated worms at Day 4, indicating that preventing iron accumulation can improve animal robustness (for all comparisons see Supplemental Tables S18-21). Lip-1 had no effect on length or volume.

We also examined whether the interventions altered early and total reproductive output when worms were treated from early adulthood/late L4 (as used in the lifespan experiments). Early fertility (first 24 hours) was not altered by either SIH or Lip-1 treatment (Figure 6D; $p>0.4$ ). Lip-1 treatment resulted in a small decrease in lifetime reproductive output (Figure 6D; $p<0.05$ ), but SIH had no effect. Early fertility in C. elegans is paramount with respect to Darwinian fitness ${ }^{39,40}$, so the reduction in lifetime fertility with Lip-1 treatment is consistent with a mild deleterious effect in early adulthood.

The effects of both interventions on movement parameters were assessed, since peak motile velocity has been previously demonstrated to correlate strongly with C. elegans healthspan and longevity ${ }^{41}$. As expected, control animals showed a steady decline in maximum velocity as they aged (Figure 6E). Treatment with SIH or Lip-1 markedly improved the maximum velocity of ageing animals (Figure 6E), with increases also in distance travelled and mean velocity (Supplemental Tables S22-27 and Figures S12-13).

\section{Discussion}

Our findings indicate that late-life dysregulation of glutathione and iron may trigger ferroptosis in $C$. elegans and that activation of this cell death signal limits lifespan. We previously determined that as $C$. 
elegans age, not only does intracellular iron accumulate but the capacity to safely sequester iron in the iron-storage protein ferritin fails. This contributes to the increased cellular fraction of $\mathrm{Fe}^{2+}$ that we observed here, leading to increased oxidative $\operatorname{load}^{2}, 16$, and providing the specific substrate for ferroptosis ${ }^{42}$. The observed depletion of glutathione during ageing further lowers the threshold for ferroptosis ${ }^{1}$.

Thus, in normal ageing, the decrease in GSH couples with an age-related increase in free $\mathrm{Fe}^{2+}$ to multiply the likelihood of ferroptosis, leading to death when combined changes in iron and GSH reach a critical threshold (Figure 7). We find that SIH and Lip-1 increase lifespan by prohibiting ferroptotic death rather than inhibiting ageing rate. We find also that late-life ferroptosis is a prominent contributor to age-related frailty. The healthspan benefits of inhibiting ferroptosis confirm that healthspan improvement need not always require a change in global ageing but can result from preventing a cause of frailty, raising an exciting conceptual prospect for therapeutic intervention.

Iron is critical to a growing organism, yet unnecessary retention might predispose towards increased frailty later in life ${ }^{7,43}$. A previous study found that iron supplementation of $C$. elegans shortened lifespan but did not measure in vivo iron levels or the fraction of $\mathrm{Fe}^{2+20}$. Our measures of chronic versus acute changes in the fraction of $\mathrm{Fe}^{2+}$ help define the organismal limits of buffering capacity at 0.3 (or 30\%). Iron deficiency causes deficits in major developmental pathways, but our interventions were delivered in adulthood to avoid any potential interference with development ${ }^{7}$. This revealed that limiting iron retention post-development was not only tolerable, but improved health and life history traits.

SIH and Lip-1 alter ageing at specific life phases and, unlike most known lifespan interventions, do not slow the rate of ageing ${ }^{37}$. They act in related but different ways to extend lifespan, with differing impacts on hazard rate and distinct effects on iron levels, life-history traits and acute glutathione depletion. Importantly, both interventions increase both lifespan and healthspan without apparent major fitness trade-offs, in contrast to those previously reported in long-lived mutants that do slow the rate of ageing $^{39,40}$.

Ageing is the principal risk factor for many major human diseases including cancer and dementia. An ancient biochemical dependence on iron may have established an inevitable liability in late life. Needless iron elevation in somatic tissue has been described in many organisms from drosophila to rodents to humans, particularly in brain ${ }^{3-5}$ and might be a universal feature of ageing. Notably, there is no excretion mechanism for systemic iron in animals ${ }^{44,45}$, highlighting that while iron is limiting for development, there has been no evolutionary pressure to regulate its accumulation in post-reproductive life. Ferroptosis plays an important role in kerbing cancer but becomes inappropriately activated in ischemia and neurodegeneration, where its inhibition holds therapeutic promise ${ }^{8-11}$. Future studies could test the hypothesis that the cancer-protective benefit of ferroptosis involves the reciprocal acceleration of ageing.

\section{Acknowledgments}

We thank Abdel Belaidi for comments on the manuscript, Nicholas Stroustrup and Walter Fontana for providing their raw data to enable validation of our temporal scaling analysis and acknowledge the Australian Synchrotron. This study was supported by grants from the Australian Research Council to AIB and GM (DP130100357 and DP180101248), University of Melbourne Research Grant Support Scheme and Miller Foundation to GM, and the Victorian Government's Operational Infrastructure Support Program. We thank the Caenorhabditis Genetics Center (CGC) supported by the US National Institutes of Health - Office of Research Infrastructure Programs (P40 OD010440) for providing $C$. elegans strains. 


\section{Author Contributions}

NLJ, AIB and GM planned the study. NLJ and GM performed the experiments with assistance from SAJ and FS. NLJ, SAJ, AS, TPS and GM analysed the data. DRR and MC provided resources. NLJ, AIB and GM wrote the manuscript with contributions from all authors.

\section{Declaration of Interests}

AIB is a paid consultant for, and has a profit share interest in, Collaborative Medicinal Development Pty Ltd. AIB and GM are inventors on patent application 15/505,384, 2017, which covers the method of reducing senescence in a mammal by reducing the concentration of non-ferritin iron. 


\section{Figures}
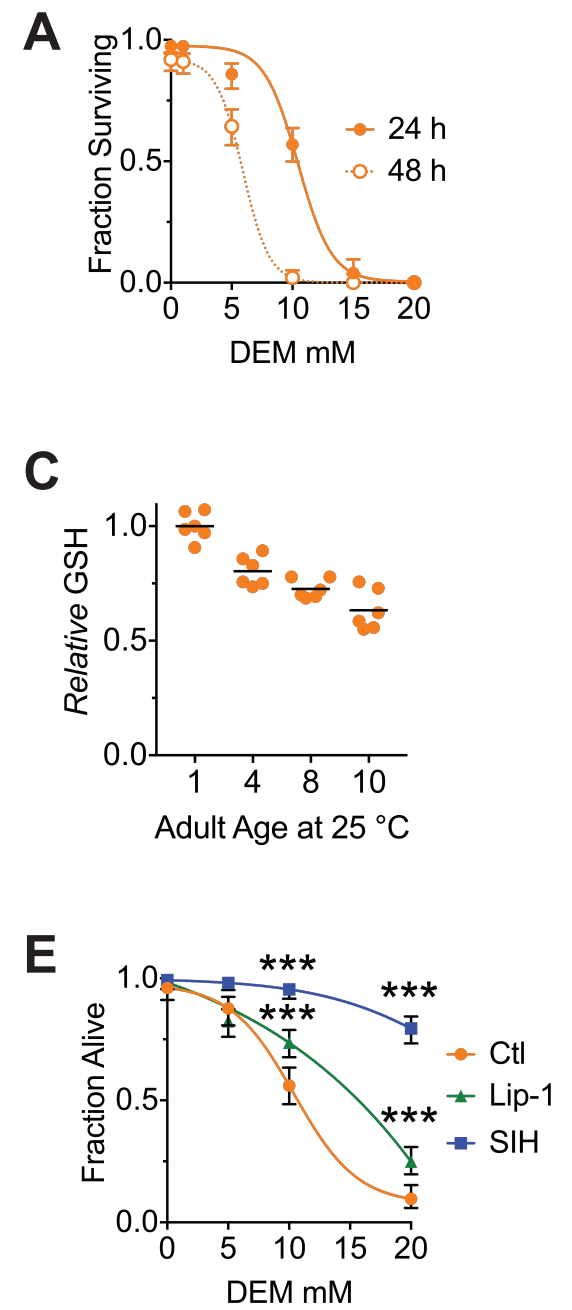

B

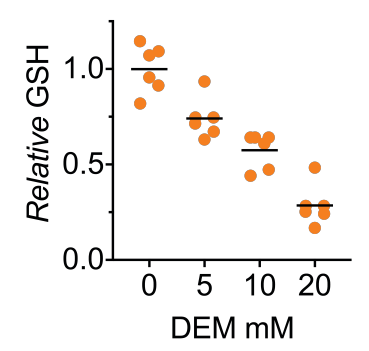

D

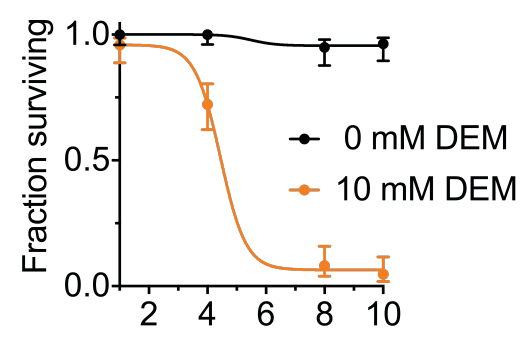

Adult age at $25^{\circ} \mathrm{C}$ prior to exposure

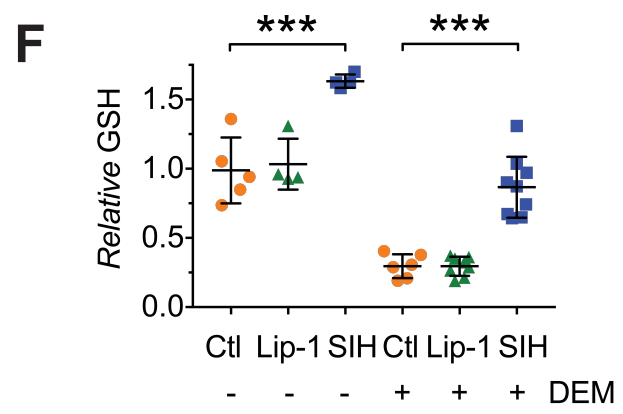

Figure 1| Both Lip-1 and SIH protect against toxicity from glutathione depletion

Treatment with DEM represents an acute stress that reduces glutathione levels and causes death, with older animals being more susceptible.

(A) Survival curves of adults following either 24- or 48-hour exposure to increasing doses of DEM. Treatment begun on Day 4 of adulthood. Shown are proportions $\pm 95 \%$ confidence intervals ${ }^{46}$, with a sigmoidal curve fitted.

(B) Total glutathione (GSH) decrease following 6 hours of DEM exposure. Day 4 adults used, with results normalized to the GSH levels in worms not exposed to DEM. Plotted are 9 independent replicates, with each estimated derived from 50 adults per measure). Linear regression $\mathrm{R}^{2}=0.98, p=0.01$

(C) Total GSH levels decrease with increased adult age in C. elegans. Each point is derived from independent replicates of 50 adults, with black lines marking the mean value. Results are normalized to the GSH levels in Day 1 worms (ANOVA: F $(3,20)=32.96, p<0.0001$; see Supplemental Table S1 for pairwise comparisons).

(D) Aged C. elegans adults become progressively more sensitive to GSH depletion by DEM. Shown are proportions $\pm 95 \%$ confidence intervals, with a sigmoidal curve fitted. 
(E) Both Lip and SIH treatment protect against lethality from DEM derived glutathione depletion. Day 4 adults, with values representing pooled data from four independent experiments $\pm 95 \%$ confidence intervals, each with a fitted sigmoidal curve. Pairwise comparisons at 10 and $20 \mathrm{mM}$ DEM were performed using Fisher's exact test; *** denotes $p<0.001$.

(F) Total glutathione levels are preserved following SIH pretreatment, but not by Lip-1. Day 4 adults were exposed to DEM (10 mM) for 6 hours, and total glutathione (GSH) assayed. Each point is derived from independent replicates of 50 adults, with black lines marking the mean value \pm SD. (ANOVA: F $(5,30)=50.97, p<0.0001$; see Supplemental Table S2 for pairwise comparisons $).{ }^{* * *}$ denotes $\mathrm{p}<0.001$ 
A

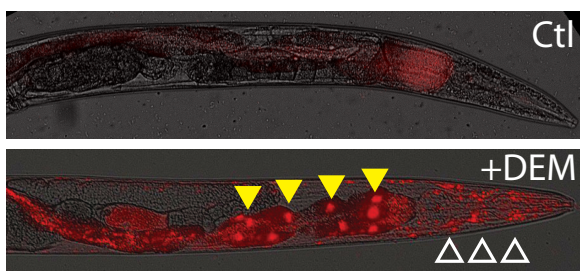

B

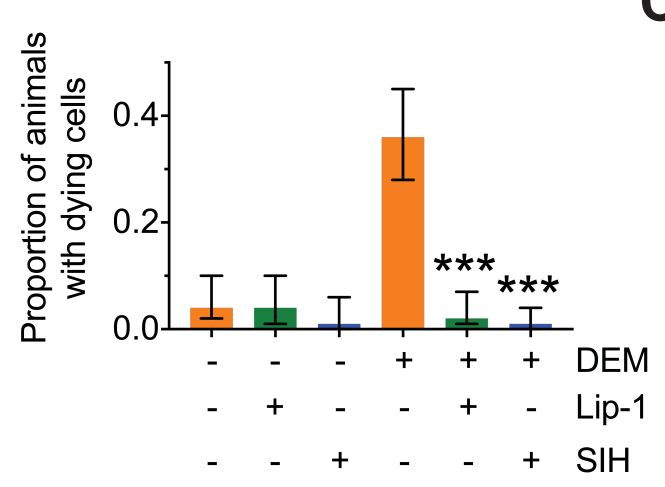

C

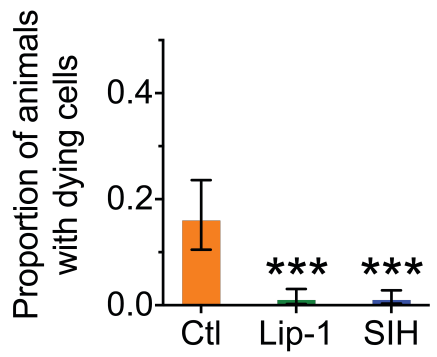

D

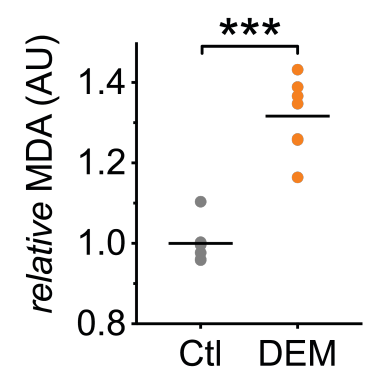

$\mathbf{F}$

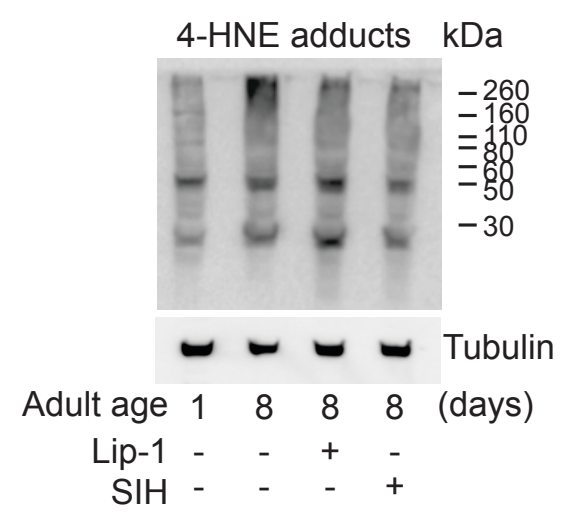

E

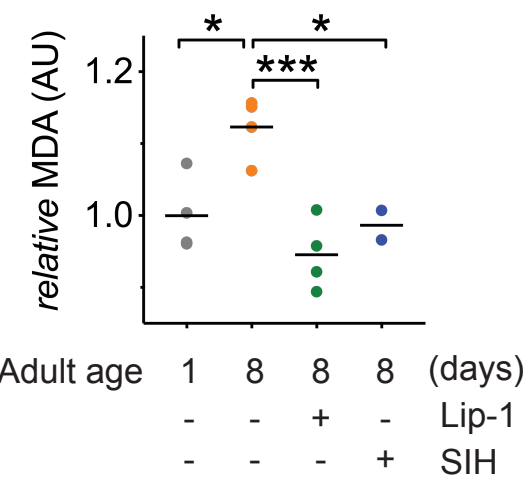

\section{Figure 2| Both Lip-1 and SIH inhibit cell death and protect against lipid peroxidation}

Measures of lipid peroxidation and cell death show an increase with age and reduction by both Lip-1 and SIH treatment.

(A) Representative propidium iodide fluorescence (red) overlay of bright field micrograph depicting dead intestinal cells (marked by nuclear signal, yellow triangles) within a live Day 4 adult treated with DEM. Smaller fluorescent puncta were also observed, consistent with neuronal cell nuclei (white unfilled triangles). Untreated Day 4 adult control animals (Ctl) showed no cell death. 
(B) Proportion of live animals at Day 4 ( $\pm 95 \%$ confidence intervals) of adulthood showing dead cell fluorescence (propidium iodide) \pm exposure to $10 \mathrm{mM}$ DEM for 24h. Cohorts of animals included: cotreatment with a vehicle control (-DEM, Ctl, $n=102$; +DEM Ctl $n=117)$, Lip-1 (-DEM, Lip-1, $n=84$; + DEM Ctl $n=106$ ) or SIH (-DEM, Ctl, $n=89$; +DEM Ctl $n=129$ ). Lip- 1 and SIH both markedly reduced the proportion of animals with dead cells after DEM treatment (z-test: Ctl vs Lip-1 $Z=6.37 * * * p<0.001$; Ctl vs SIH Z=7.24, *** $\mathrm{p}<0.001)$.

(C) Proportion of live animals at Day 8 ( $\pm 95 \%$ confidence intervals) of adulthood showing dead cell fluorescence (propidium iodide). Co-treatment with Lip-1 $(n=234)$ or SIH $(n=308)$ markedly reduced the proportion of animals with dying cells compared to vehicle control (Ctl, $n=119$; $z$-test: Ctl vs Lip-1 $\mathrm{Z}=5.67 * * * \mathrm{p}<0.001$; Ctl vs $\mathrm{SIH} \mathrm{Z}=6.28$, *** $\mathrm{p}<0.001)$.

(D) Levels of the lipid peroxidation end product malondialdehyde (MDA) increases in C. elegans following acute glutathione depletion by $20 \mathrm{mM}$ DEM exposure for $6 \mathrm{~h}$. MDA levels are shown as values normalized against the mean of untreated Day 4 Adults $(\mathrm{Ctl})$ for independent samples. (Ctl vs +DEM, unpaired 2-tailed t-test $* * * \mathrm{p}<0.001)$

(E) Malondialdehyde (MDA) increases in aged C. elegans (Day 1 vs Day 8 adults, ANOVA * $<<0.05$ ). Treatment with either Lip-1 (Day 8 vs Day $8+$ Lip-1 adults, ANOVA *** $\mathrm{p}<0.001$ ) or SIH (Day 8 vs Day $8+$ SIH adults, ANOVA * $\mathrm{p}<0.05)$ reduces levels of MDA. Data represent independent samples with values normalized against the mean of untreated Day 1 Adults.

(F) Representative immunoblot against 4-HNE protein adducts comparing Day 1 control and Day 8 control adults and aged adults treated with Lip-1 and SIH with corresponding tubulin blot below (representative of triplicate experiments). The relative intensity of the bands show an age-related increase that is ameliorated by Lip-1 and SIH. 


\section{A}
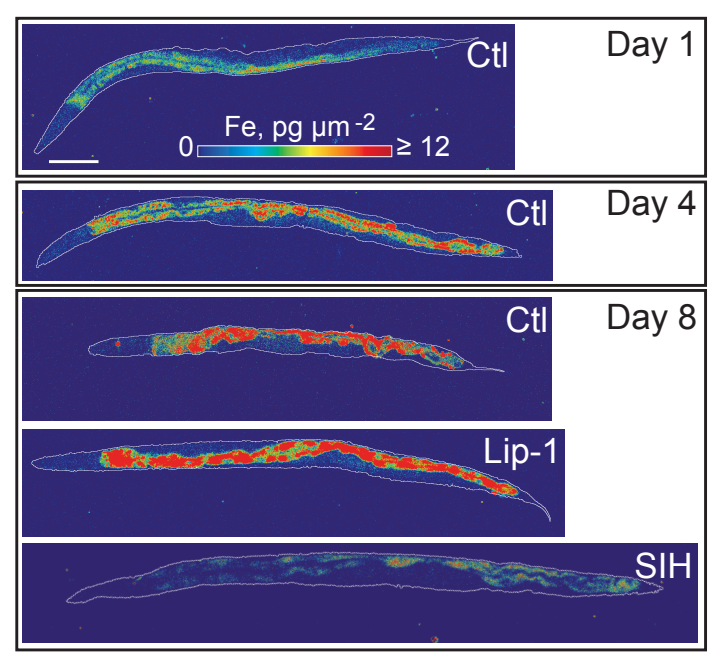

B

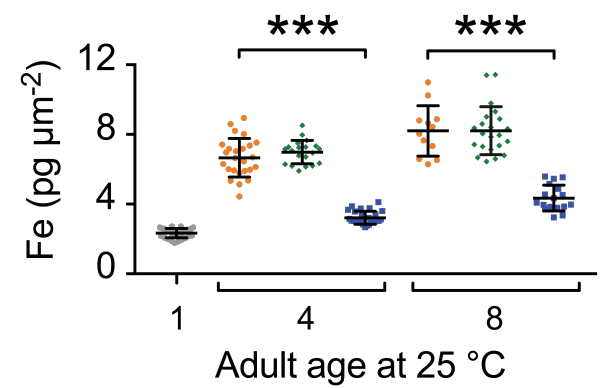

C

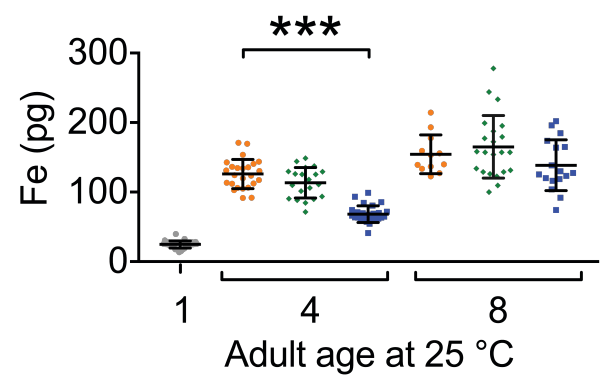

D

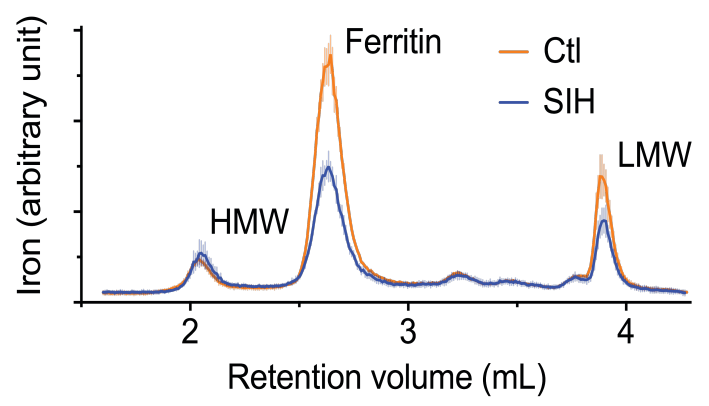

Figure 3| Effects of Lip-1 and SIH on iron levels and distribution in C. elegans.

In all panels, vehicle control $(0.5 \% \mathrm{v} / \mathrm{v}$ DMSO, $\mathrm{Ctl})$ treated worms are shown in orange, Lip- 1 treated (200 $\mu \mathrm{M}$ Lip-1) are green and SIH treated $(250 \mu \mathrm{M} \mathrm{SIH})$ are blue.

(A) Representative X-ray fluorescence microscopy maps of tissue iron ( $\mathrm{Fe}$ ) reported as areal density (pg $\mu \mathrm{m}^{-2}$ ) for a first day adult (Day 1) and animals treated for four days (Day 4) and eight days (Day 8) with vehicle control (Ctl), Lip-1 or SIH at $25^{\circ} \mathrm{C}$. Scale bar $=50 \mu \mathrm{m}$. 
(B) Plot of mean areal density for iron $\left(\mathrm{pg} \mu \mathrm{m}^{-2}\right.$ ) for all treatment cohorts aged at $25{ }^{\circ} \mathrm{C}$. The starting population (Day 1) is shown in grey. The control cohort (orange) shows an age related increase in total iron (as previously observed ${ }^{2}$ ). The Lip-1 group (green) has similar iron levels across each age, whereas the SIH cohort (blue) has markedly less total iron (ANOVA: $\mathrm{F}(6,148)=171.3, p<0.0001$; see Supplemental Table $\mathbf{S 3}$ for sample summary and Table $\mathbf{S 4}$ for pair-wise comparisons). Each data point represents a value from a single $C$. elegans adult, with mean $\pm \mathrm{SD}, * * * p<0.001$.

(C) Plot of total body iron (pg) for treated C. elegans cohorts aged at $25{ }^{\circ} \mathrm{C}$. Each data point represents a value from a single $C$. elegans adult, with mean $\pm \mathrm{SD}$. All treatments have increased total iron across age with SIH treated (blue) worms retaining significantly less iron than control (red) and Lip-1 (green) treated worms at Day 4 (ANOVA: $\mathrm{F}(6,148)=97.3$, *** $p<0.0001$; see Supplemental Table S5 for sample summary and Table S6 for pair-wise comparisons)

(D) Native, size-exclusion chromatography of iron-macromolecular complexes from 10 day old adults treated with vehicle control (Ctl, shown in orange) or SIH treated cohorts (shown in blue). The means \pm $\mathrm{SD}$, from three independent biological replicates, are plotted. The three major peaks include unaltered high molecular weight complexes (HMW, $>1 \mathrm{MDa}, \sim 2.2 \mathrm{~mL}$ retention volume), ferritin bound iron $(\sim 2.7$ $\mathrm{mL}$ retention volume; previously identified as $\mathrm{FTN}-2^{2}$; area under the peak decreased by $\sim 53 \%$ relative to $\mathrm{Ctl}$ ) and low molecular weight iron complexes (LMW, $<30 \mathrm{kDa}, \sim 3.9 \mathrm{~mL}$ retention volume, decreased $\sim 47 \%$ relative to $\mathrm{Ctl})$. 

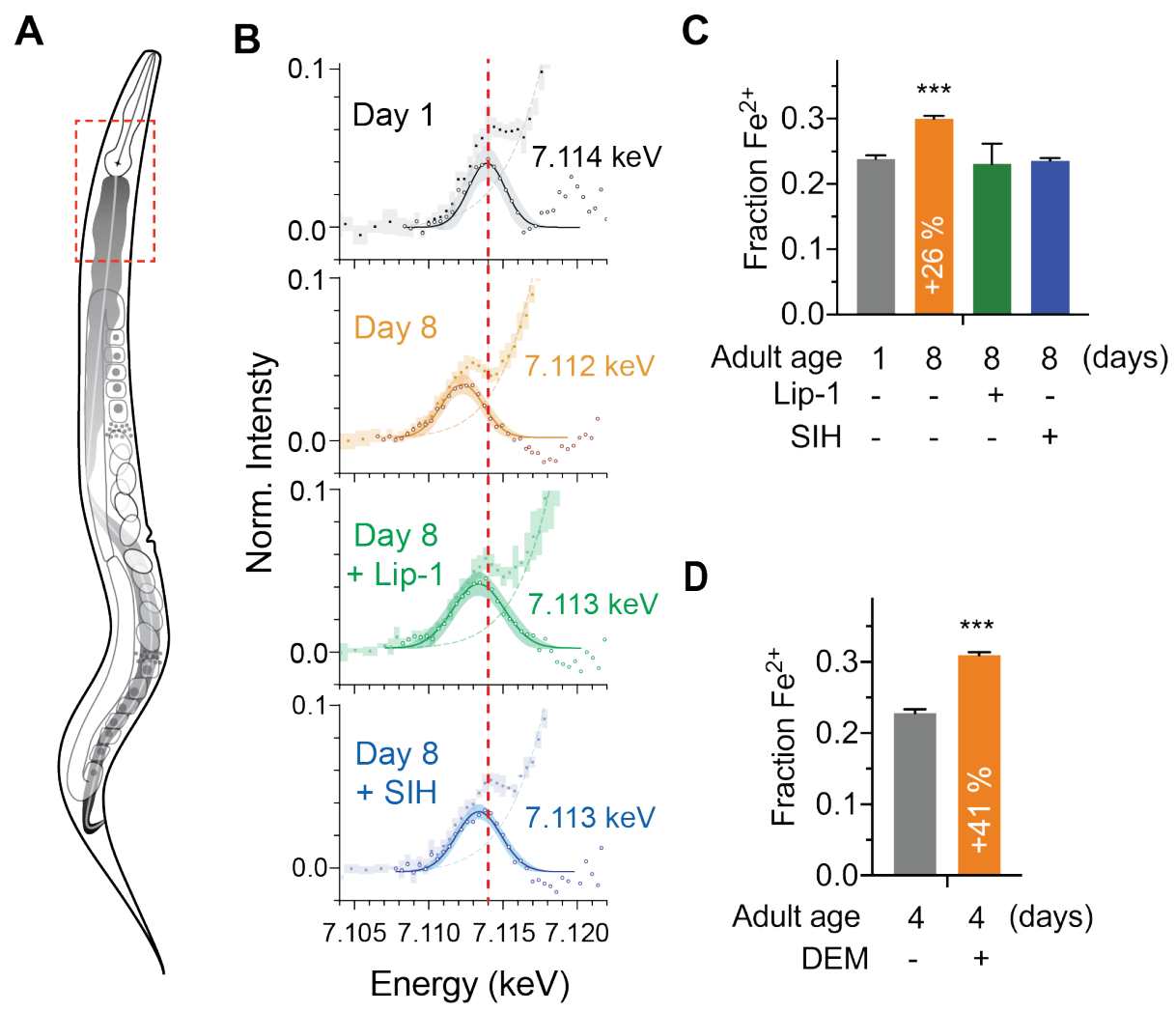

Figure 4| Effects of ageing, glutathione depletion, SIH and Lip-1 on pro-ferroptotic Fe ${ }^{2+}$ levels. $\varphi$ XANES evaluation of the $\mathrm{Fe}^{2+}$ fraction in vivo within intact animals.

(A) Schematic highlighting the anatomy of an adult hermaphrodite C. elegans with the intestine shaded in grey. Dashed box is indicative of the region of animals selected for $\varphi$ XANES.

(B) $\mathrm{Fe}^{2+}$ synchrotron microscopy. $\varphi$ XANES imaging allowed extraction of the normalised Fe K-edge XANES spectra (coloured circles) from the anterior intestine of Day $1(n=6)$ and Day 8 control $(n=4)$, SIH treated $(n=4)$ and Lip-1 treated $(n=5)$ worms. Averaged spectra for each group are shown along with $95 \%$ confidence intervals (shading). The pre-edge region, following subtraction of the rising edge (dashed line), highlights changes in the intensity and position of the $1 \mathrm{~s} \rightarrow 3 \mathrm{~d}$ transition. The extracted data (empty circles) and fitted Gaussian (solid lines; shading represents the 95\% CI) are superimposed to determine the centroid values for the pre-edge peak, from which the $\mathrm{Fe}^{2+}$ fraction is derived. Changes in the first derivative of the Fe K-edge XANES (Supplemental Figure S3) reflect variation in the intensity of the $1 \mathrm{~s} \rightarrow 4 \mathrm{~s}$ and $1 \mathrm{~s} \rightarrow 4 \mathrm{p}$ transitions. The relative intensity of these features was used to estimate the proportion of $\mathrm{Fe}^{2+}$ iron in the specimens. For reference, the red line through all spectra denotes the centroid of the Day 1 adults at $7.114 \mathrm{keV}$.

(C) The proportional change in fractional $\mathrm{Fe}^{2+}$ contribution for spectrum in the aged (Day 1 versus Day 8 adults) and treated (Lip-1 and SIH, from panel B) specimens is indicated, along with 95\% confidence interval. Changes in the first derivative of the Fe K-edge XANES (Supplemental Figure S3) was used to infer variation in the intensity of the $1 \mathrm{~s} \rightarrow 4 \mathrm{~s}$ and $1 \mathrm{~s} \rightarrow 4 \mathrm{p}$ transitions and the relative intensity of these features was then used to estimate the proportion of $\mathrm{Fe}^{2+}$ iron.

(D) The proportional change in fractional $\mathrm{Fe}^{2+}$ contribution for Day 4 adults treated with $(n=4)$ and without acute glutathione depletion via $\operatorname{DEM}(n=4)$ is indicated, along with $95 \%$ confidence interval. 
A

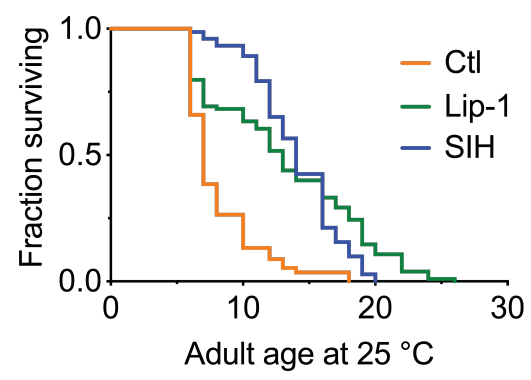

B

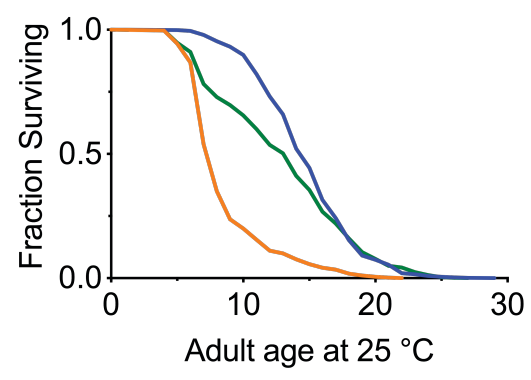

C

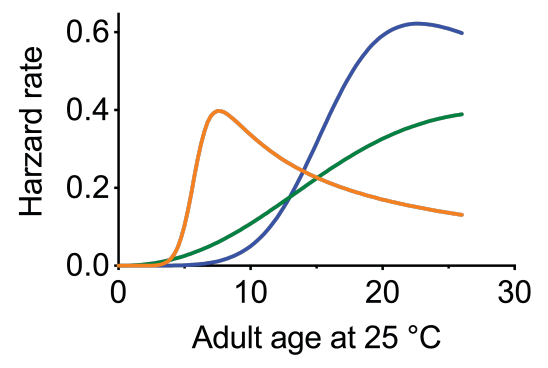

Figure 5| Inhibiting ferroptosis extends Caenorhabditis elegans lifespan.

Treatment with both Lip-1 and SIH extend lifespan.

(A) Representative Kaplan-Meier survival curve from C. elegans treated with vehicle control (Ctl, median survival 7 days, death event $n=88$ ); Lip-1 (median survival 13 days, Log-rank test $p<0.001, n$ $=103$ ) and $\mathrm{SIH}$ (median survival 14 days, $p<0.001, n=71$ ) at $25^{\circ} \mathrm{C}$.

(B) Survival curve derived from pooled data from all eight replicate experiments (Ctl $n=709$, Lip- $1 n=$ 809, and SIH $n=720$; see Supplemental Table S8) at $25^{\circ} \mathrm{C}$.

(C) Plot of hazard (mortality) rate against age at $25{ }^{\circ} \mathrm{C}$, derived from meta-analysis of pooled data (presented in b). Both SIH and Lip-1 alter mortality rates relative to control populations and are also distinct from each other. (see Supplemental Tables S13-14). 


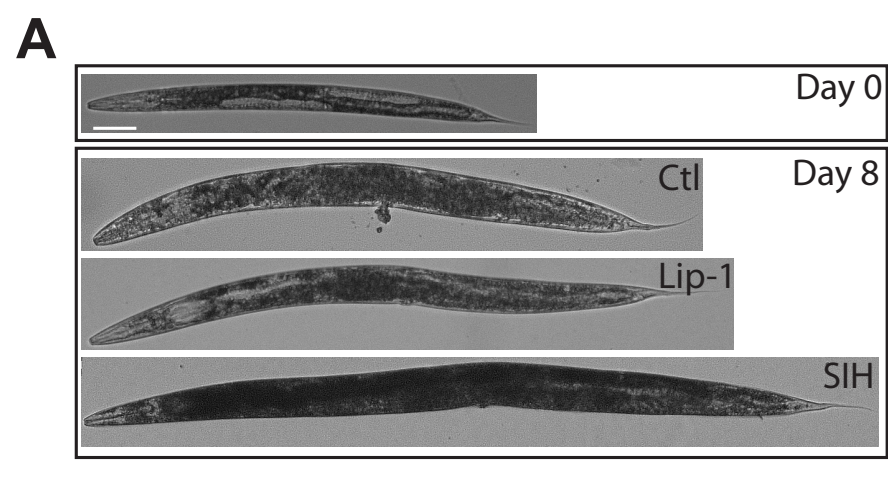

B
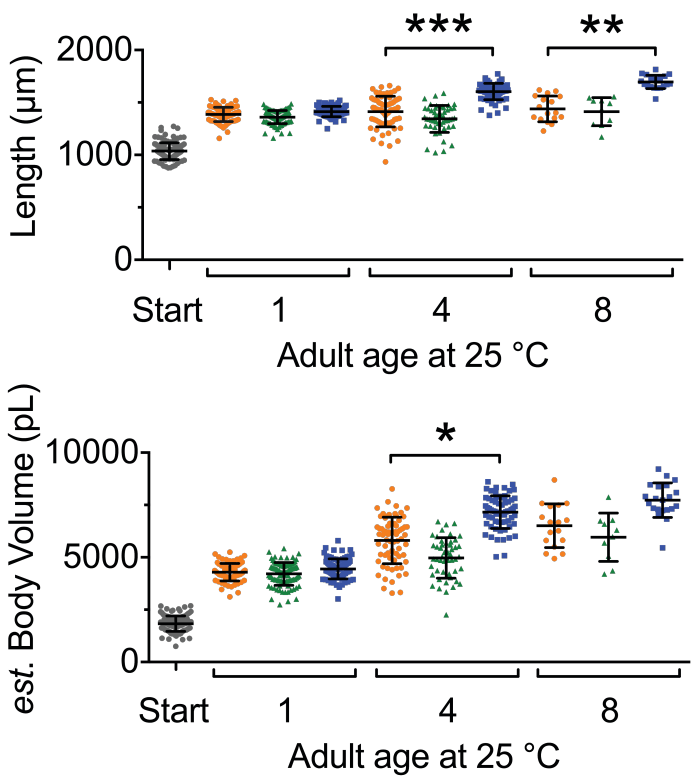

D

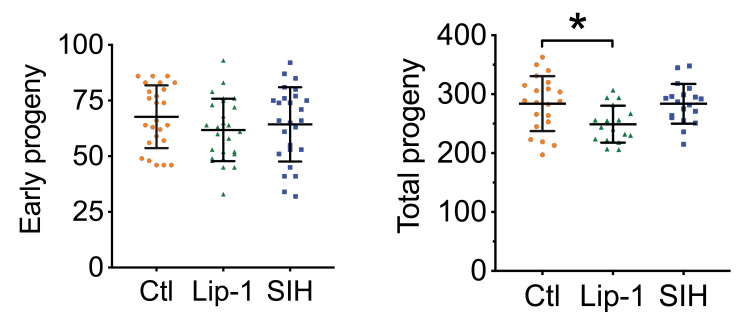

$\mathbf{E}$

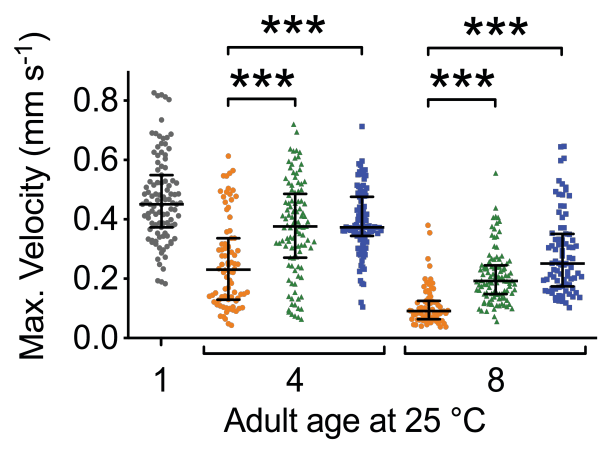

Figure 6| Lip-1 and SIH blocks frailty in C. elegans.

In all panels, vehicle control $(0.5 \% \mathrm{v} / \mathrm{v} \mathrm{DMSO}, \mathrm{Ctl})$ treated worms are shown in orange, Lip-1 treated $(200 \mu \mathrm{M}$ Lip-1) are green and SIH treated $(250 \mu \mathrm{M}$ SIH) are blue. Significant differences between treatments are highlighted where $*$ indicates $p<0.05, * *$ indicates $p<0.01$ and $* * *$ indicates $p<0.001$. 
(A) Micrograph of an adult C. elegans on the first day of treatment (Day 0) and animals treated with Ctl, Lip-1 or SIH for eight days at $25^{\circ} \mathrm{C}$ (Day 8). Scale bar $=50 \mu \mathrm{m}$.

(B) Estimates of adult body length (in $\mu \mathrm{m}$ ), showing SIH treated animals (blue) have longer average body length compared to age matched control (orange)or Lip-1 (green) treated populations (KruskalWallis ANOVA: $\mathrm{H}(10)=432.6, p<0.0001$; see Supplemental Table S18 for sample summary and S19 for pair-wise comparisons). Start (grey) represents the beginning population of L4/young adults grown from egg at $25{ }^{\circ} \mathrm{C}$ for 48 hours prior to transfer to treatment plates. Each point represents an individual worm, with mean and error bars representing standard deviation (SD)

(C) Estimated adult body volume (in $\mathrm{pL}$ ), showing increased body volume with adult age for all groups (Kruskal-Wallis ANOVA: H(10)=489, $p<0.0001$; see Supplemental Table S20 for sample summary and Table S21 for pair-wise comparisons), with SIH treated animals having even greater body volume. Each point represents an individual worm, with mean \pm SD.

(D) Early fertility (first 24 hours) and total reproductive output are unaltered when vehicle control (Ctl) treated cohorts are compared to Lip-1 or SIH treated animals at $25{ }^{\circ} \mathrm{C}$. Each data point represents an estimate from a single $C$. elegans adult, with mean $\pm \mathrm{SD}$ (ANOVA: Early fertility $\mathrm{F}(2,74)=0.996, p=0.37$; Total fertility $\mathrm{F}(2,57)=4.89, p=0.011)$.

(E) Estimates of maximum velocity achieved by aged and treated cohorts of $C$. elegans. Treatment with either Lip-1 or SIH attenuates the age-related decline in maximum velocity (Kruskal-Wallis ANOVA: $\mathrm{H}(7)=298.5, p<0.0001$; see Supplemental Table $\mathbf{S 2 2}$ for sample summary and Table S23 for pairwise comparisons). Each data point represents an estimate from a single C. elegans adult, with median \pm interquartile range. Equivalent analyses of mean velocity and total distance travelled (and how these data correlate) are shown in Supplemental Figures S12-14 and Supplemental Tables S24-27). 


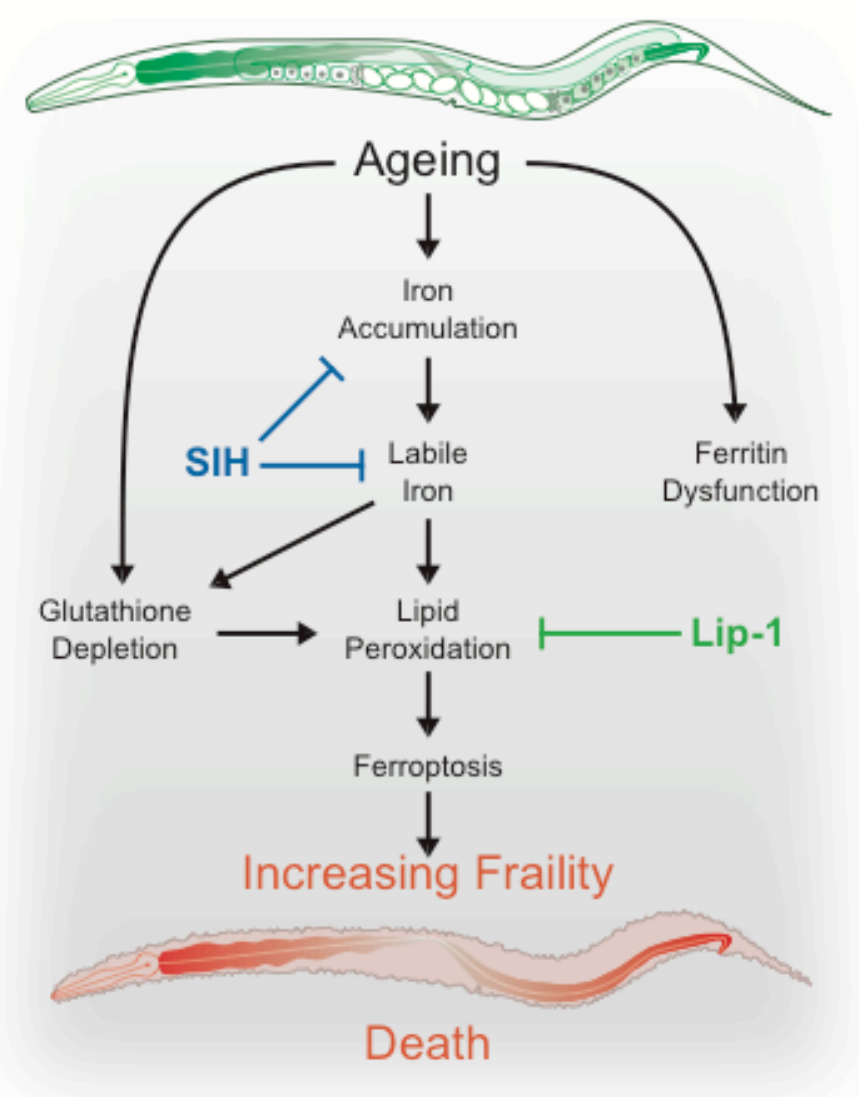

Figure 7| Schematic overview. During normal ageing iron unnecessary accumulates. The safe storage of surplus iron in ferritin begins to fail in late life, causing a corresponding elevation of reactive, 'labile' iron. In combination with falling glutathione levels there is increased risk of ferroptotic cell death, via lipid peroxidation signals. These cell death events increase frailty and ultimately shorten organism lifespan. These pharmacological interventions potentially represent targets to improve late life vigor and fitness. 


\section{METHODS}

Strains. Wild type (strain N2) and the temperature sensitive-sterile strain TJ1060: spe-9(hc88); fer15(b26) were obtained from the Caenorhabditis Genetics Center. The wild type strain was maintained at $20{ }^{\circ} \mathrm{C}$ on standard nematode growth media (NGM) ${ }^{47}$ and aged at $20{ }^{\circ} \mathrm{C}$ or $25^{\circ} \mathrm{C}$ as required. TJ 1060 was maintained at $16^{\circ} \mathrm{C}$ and also aged at $20^{\circ} \mathrm{C}$ or $25^{\circ} \mathrm{C}$ as required. TJ1060 was predominately used to remove the inconvenience of progeny production and can be regarded as a proxy for wild type.

Compounds. Compounds used in this study include:

- Diethyl maleate (DEM) obtained from Sigma-Aldrich.<smiles>CCOC(=O)/C=C\C(=O)OCC</smiles>

- Liproxstatin (Lip-1; N-[(3-chlorophenyl) methyl]-spiro[piperidine-4,2'(1'H)-quinoxalin]-3'amine) obtained from the laboratory of Marcus Conrad (initially) and subsequently ApexBio Tech LLC.<smiles>Clc1cccc(CNC2=Nc3ccccc3NC23CCNCC3)c1</smiles>

- Salicylaldehyde isonicotinoyl hydrazone (SIH) obtained from the laboratory of Des Richardson (University of Sydney).<smiles>O=C(N/N=C/c1ccccc1O)c1ccncc1</smiles>

- $\quad$ SIH precomplexed with iron as $\mathrm{Fe}(\mathrm{SIH})_{2} \mathrm{NO}_{3}$.

Glutathione depletion. Diethyl maleate (DEM; Sigma-Aldrich) was added to neat DMSO and added to molten NGM at $55^{\circ} \mathrm{C}$ to a final concentration of $5,10,15$ or $20 \mathrm{mM} \mathrm{DEM}$ and $0.5 \% \mathrm{v} / \mathrm{v}$ DMSO. Plates were seeded with OP50 and used within 24 hours. As above, data was collected at $25( \pm 1){ }^{\circ} \mathrm{C}$ using the temperature sensitive-sterile strain TJ1060. A synchronous population was obtained by transferring egglaying adults to fresh plates at $16{ }^{\circ} \mathrm{C}$ for 2-3 hours. The adults were removed and the plates with eggs then transferred to $25^{\circ} \mathrm{C}$ to ensure sterility. After 48 hours at $25^{\circ} \mathrm{C}$, when worms were at the late L4/young adult stage, 25-35 nematodes were transferred to fresh plates containing either vehicle control, $250 \mu \mathrm{M}$ SIH, or $200 \mu \mathrm{M}$ Lip-1. Worms were aged at $25^{\circ} \mathrm{C}$ for a further 4 days and then transferred to DEM plates. Survival, determined by touch-provoked movement, was scored at 24 and 48 hours after exposure to DEM.

Ageing studies were also undertaken to determine changes with age of both survival after DEM exposure and basal glutathione levels. Initial populations were obtained as describe above, with worms aged on standard NGA plates.

Quantification of total glutathione. Measurement of total glutathione per worm was based on established protocols and is based on a kinetic spectrophotometric assay using the reaction between GSH and 5,5'-dithio-bis (2-nitrobenzoic acid) (DTNB) measured at $412 \mathrm{~nm}{ }^{48,49}$. All reagents were freshly 
prepared prior to the assay and for each estimate 50 adults were collected in $200 \mu \mathrm{L}$ of S-basal ${ }^{47}$ in 1.7 $\mathrm{ml}$ microfuge tubes. Animals were washed twice in S-basal, pelleted via centrifugation and total volume reduced to $20 \mu \mathrm{L}$. A $50 \mu \mathrm{L}$ aliquot of Extraction Buffer was added, then the samples were frozen in Liquid $\mathrm{N}_{2}$ and store at $-80^{\circ} \mathrm{C}$ until required. Extraction buffer consisted of $6 \mathrm{mg} / \mathrm{mL} 5$-sulfosalicylic acid dehydrate, $0.1 \% \mathrm{v} / \mathrm{v}$ Triton X-100 and Complete, EDTA-free Proteinase inhibitor cocktail (Roche) in KPE buffer (0.1 M potassium phosphate buffer and $5 \mathrm{mM}$ EDTA at $\mathrm{pH}$ 7.5).

Samples were homogenized with a Bioruptor Next Gen (Diagenode) bath sonicator, set on HIGH and cooled to $4{ }^{\circ} \mathrm{C}$, using 10 cycles of 10 seconds ON and 10 seconds OFF. Supernatant was collected following a $14 \mathrm{~K} \mathrm{x} g$ spin at $4{ }^{\circ} \mathrm{C}$. Assays were performed in 96 well microplates (clear polystyrene, flatbottomed, Greiner bio-one), in a total volume of $200 \mu \mathrm{L}$ per well. To each well was added $50 \mu \mathrm{L}$ of lysate supernatant, $50 \mu \mathrm{L}$ of milli-Q $\mathrm{H}_{2} \mathrm{O}$ and then $100 \mu \mathrm{L}$ of GA buffer (NADPH $400 \mu \mathrm{M}$, glutathione reductase $1 \mathrm{U} / \mathrm{mL}$ and $0.3 \mathrm{mM}$ DTNB in KPE buffer diluent). Reactions were incubated for $1-2 \mathrm{~min}$ at room temperature and then absorbance measured at $412 \mathrm{~nm}$ for $10 \mathrm{~min}$ with $1 \mathrm{~min}$ interval using a Powerwave plate spectrophotometer (BioTek). The rate of change in absorbance per minute is linearly proportional to the total concentration of GSH. Total GSH in the samples was interpolated from using linear regression from a standard curve of known GSH concentrations $(0$ to $1 \mu \mathrm{M})$ run in tandem. Within experiment results are presented as relative glutathione levels, where results are normalized to the mean of the starting population.

Lipid peroxidation. Measurement of malondialdehyde (MDA) was performed using a Thiobarbituric acid reactive substances (TBARS) assay kit (10009055, Caymen Chemical) as per manufacturer instructions using reduced reaction volumes of $1 \mathrm{~mL}$. For $C$. elegans samples with acute glutathione depletion, Day 1 adults were treated with and without $20 \mathrm{mM}$ DEM for $6 \mathrm{~h}$ at $25{ }^{\circ} \mathrm{C}$ prior to collection. For ageing, animals were aged at $25^{\circ} \mathrm{C}$ and treated with Lip-1 or SIH as previously described. Replicate samples were collect, washed twice in S-basal, pelleted by centrifugation. Following removal of excess buffer samples $(\sim 40 \mu \mathrm{L})$ were frozen in liquid- $\mathrm{N}_{2}$ and stored at $-80^{\circ} \mathrm{C}$ until needed. Samples were then homogenized via a Bioruptor bath sonicator (Diagenode, set on 'high power' with 10 cycles of 10s pulses with a 10 s pause between pulses, at $4{ }^{\circ} \mathrm{C}$ ), then centrifuged at $21,500 \mathrm{xg}$ at $4{ }^{\circ} \mathrm{C}$ for $30 \mathrm{~min}$ and the supernatant retained. The concentration of protein was determined using a BCA assay kit (Bio-Rad) and equivalent aliquots of 20-25 $\mu \mathrm{g}$ total protein used for subsequent measurements.

Analysis of Hydroxynonenal (4-HNE) protein adducts was also used as a proxy for lipid peroxidation. Duplicate samples of 50 and 200 worms were collected and washed twice in S-basal, pelleted by centrifugation and the supernatant discarded. These samples $(\sim 30 \mu \mathrm{L})$ were frozen in liquid- $\mathrm{N}_{2}$ and stored at $-80^{\circ} \mathrm{C}$ until needed. To each sample an $10 \mu \mathrm{L} 4 \mathrm{x}$ Bolt LDS sample buffer (Invitrogen) and $3 \mu \mathrm{L}$ TCEP (Invitrogen) was added and the sample heated to $95^{\circ} \mathrm{C}$ for $10 \mathrm{~min}$. Lysates were loaded onto NuPAGETM 4-12\% Bis-Tris acrylamide gels (1.0 mm, 10-well, Invitrogen), electrophoresed with MES running buffer and then transferred onto $0.45 \mu \mathrm{m}$ PVDF membrane by electroblot using a Mini Blot module (Invitrogen). 4-HNE protein adducts were detected by an anti 4-HNE protein adduct antibody (1:2000, AB5605, Millipore) in Tris-buffer saline with 5\% skim milk, and ECL (GE Healthcare). The membranes were stripped using a 1x ReBlot Strong Antibody Stripping Solution (Merck) for $15 \mathrm{~min}$, reprobed for tubulin using an anti-Tubulin antibody (1:10,000, T6074, Sigma-Aldrich).

Visualization of cell death. The red-fluorescent propidium iodide (PI), was used to visualize dead cells within live $C$. elegans after DEM treatment and during ageing. Populations were incubated for $24 \mathrm{~h}$ at $25^{\circ} \mathrm{C}$ with PI (a $10 \mu \mathrm{L}$ volume of $0.25 \mathrm{mg} / \mathrm{mL}$ solution added to the bacterial lawn on $50 \mathrm{~mm}$ NGM plates) prior to the described age or with concurrent exposure to $10 \mathrm{mM} \mathrm{DEM} \mathrm{(as} \mathrm{described} \mathrm{above)} \mathrm{and}$ PI. For ageing experiments, animals were visualized at Day 6 and Day 8. Cohorts of live animals (i.e. showing spontaneous or touch-provoked movement) were isolated and mounted under glass coverslips on $2 \%$ agarose pads without anesthetic. Imaging were captures with on a Leica DMI3000B inverted microscope, DsRed filter set and a DFC 3000G digital.

Liquid chromatography-inductively coupled plasma mass spectrometry. Liquid chromatography was performed using established protocols ${ }^{2}$. Briefly, samples of aged C. elegans were lysed using a Bioruptor Next Gen (Diagenode) bath sonicator set on HIGH and cooled to $4{ }^{\circ} \mathrm{C}$ using 10 cycles of 10 sec ON and $10 \mathrm{sec}$ OFF, in a 1:1 volume ratio of Tris-buffered saline ( $\mathrm{pH}$ 8.0) with added proteinase inhibitors (EDTA-free; Roche). Sample homogenization was confirmed by microscopic inspection. Lysates were then centrifuged for $15 \mathrm{~min}$ at $175,000 \mathrm{~g}$ at $4{ }^{\circ} \mathrm{C}$. The supernatant was removed and total 
protein concentration in the soluble fraction was determined using a NanoDrop UV spectrometer (Thermo Fisher Scientific) before being transferred to standard chromatography vials with polypropylene inserts (Agilent Technologies) and kept at $4{ }^{\circ} \mathrm{C}$ on a Peltier cooler for analysis. Size exclusion chromatography-inductively coupled plasma-mass spectrometry was performed using an Agilent Technologies 1100 Series liquid chromatography system with a BioSEC 5 SEC column ( $5 \mu \mathrm{m}$ particle size, $300 \AA$ pore size, I.D. $4.6 \mathrm{~mm}$, Agilent Technologies) and 7700x Series ICP-MS as previously described $^{50}$. A buffer of $200 \mathrm{mM} \mathrm{NH}_{4} \mathrm{NO}_{3}$ was used for all separations at a flow rate of $0.4 \mathrm{~mL} \mathrm{~min}^{-1}$. A total of $50 \mu \mathrm{g}$ of soluble protein was loaded onto the column by manually adjusting the injection volume for each sample. Mass-to-charge ratios $(\mathrm{m} / \mathrm{z})$ for phosphorus (31) and iron (56) were monitored in time resolved analysis mode.

Plots of the mean ( \pm standard deviation) of three independent biological replicates are shown. Integration of the three major peaks was performed using Prism (ver. 7 for Mac OS X, Graphpad).

\section{X-ray Fluorescence Microscopy}

Sample preparation - Elemental mapping. Specimens were prepared for XFM using previously described protocols ${ }^{51,52}$. Briefly, adult $C$. elegans were removed from NGM, washed four times in excess S-basal $\left(0.1 \mathrm{M} \mathrm{NaCl} ; 0.05 \mathrm{M} \mathrm{KHPO}_{4}\right.$ at $\mathrm{pH}$ 6.0), briefly in ice-cold $18 \mathrm{M} \Omega$ resistant de-ionized $\mathrm{H}_{2} \mathrm{O}$ (Millipore) and twice in ice-cold $\mathrm{CH}_{3} \mathrm{COONH}_{4}(1.5 \% \mathrm{w} / \mathrm{v})$. Samples were transferred onto $0.5 \mu \mathrm{m}$-thick silicon nitride $\left(\mathrm{Si}_{3} \mathrm{~N}_{4}\right)$ window (Silson), excess buffer wicked away and then the slide was frozen in liquid nitrogen $\left(\mathrm{N}_{2}\right)$-chilled liquid propane using a KF-80 plunge freezer (Leica Microsystems). The samples were lyophilised overnight at $-40{ }^{\circ} \mathrm{C}$ and stored under low vacuum until required.

Elemental mapping. The distribution of metals was mapped at the X-ray Fluorescence Microscopy beamline at the Australian Synchrotron ${ }^{53}$ using the Maia detector system ${ }^{54}$. The distribution of elements with atomic number $<37$ were mapped using an incident beam of $15.6 \mathrm{keV}$ X-rays. This incident energy allowed clear separation of X-ray fluorescence (XRF) peaks from the relatively intense elastic and inelastic scatter. The incident beam $\left(\sim 1.71 \times 10^{9}\right.$ photons s$\left.{ }^{-1}\right)$ was focussed to approximately $2 \times 2 \mu \mathrm{m}^{2}$ $(\mathrm{H} \times \mathrm{V}, \mathrm{FWHM})$ in the sample plane and the specimen was continuously scanned through focus $(1 \mathrm{~mm}$ $\mathrm{sec}^{-1}$ ). The resulting XRF was binned in $0.8 \mu \mathrm{m}$ intervals in both the horizontal and vertical giving virtual pixels spanning $0.64 \mu \mathrm{m}^{2}$ of the specimen probed with a dwell time of $8 \mu \mathrm{sec}$. XRF intensity was normalized to the incident beam flux monitored with a nitrogen filled ionization chamber with a $27 \mathrm{~cm}$ path length placed upstream of the focusing optics. Three single-element thin metal foils of known areal density (Mn $18.9 \mu \mathrm{g} \mathrm{cm}^{-2}$, Fe $50.1 \mu \mathrm{g} \mathrm{cm}^{-2}$ and Pt $42.2 \mu \mathrm{g} \mathrm{cm}^{-2}$, Micromatter, Canada) were used to calibrate the relationship between fluorescence flux at the detector and elemental abundance. Dynamic Analysis, as implemented in GeoPIXE 7.3 (CSIRO), was used to deconvolve the full XRF spectra at each pixel in the scan region to produce quantitative elemental maps ${ }^{55}$. This procedure includes a correction for an assumed specimen composition and thickness, in this case $30 \mu \mathrm{m}$ of cellulose. Though unlikely to exactly match the actual sample characteristics, deviations from these assumptions are not significant for the results presented in this study as the effects of beam attenuation and self-absorption on calcium and iron XRF are negligible for a dried specimen of this type and size ${ }^{56}$.

Elemental quantification and image analysis. Analysis of elemental XRF maps was performed using a combination of tools native to GeoPIXE and ImageJ ${ }^{57}$. Incident photons inelastically scattered (Compton scatter) from the sample detail the extent and internal structure of individual C. elegans. The differential scattering power of the specimens and substrate allowed individual animals (or parts thereof) to be identified as regions of interest (ROI; Supplemental Figure S1) facilitating analysis of elemental content on a 'per worm' basis. This segmentation of each elemental map was achieved using the histogram of pixel intensities from Compton maps to locate the clusters within the image. ROIs composed of $<10,000$ pixels were deemed to be so small that their elemental content was not reflective of the elemental content of whole animals and so these were excluded from the analysis. The 'non-worm' region of each scan was used to calculate the value specimen elemental content was distinguishable from background noise, i.e. the critical value as defined by ${ }^{58}$. The background corrected elemental maps were used to establish the areal densities and the total mass of each element associated with individual ROIs.

Sample preparation - $\varphi$ XANES Imaging. Adult $C$. elegans were removed from NGM, washed four times in excess ice-cold S-basal $\left(0.1 \mathrm{M} \mathrm{NaCl} ; 0.05 \mathrm{M} \mathrm{KHPO}_{4}\right.$ at $\mathrm{pH}$ 6.0). Samples were transferred onto 
$0.5 \mu$ m-thick silicon nitride $\left(\mathrm{Si}_{3} \mathrm{~N}_{4}\right)$ window (Silson), excess buffer wicked away and then the slide was frozen in situ under a laminar stream of $100^{\circ} \mathrm{K}$ dry nitrogen $\left(\mathrm{N}_{2}\right)$ gas.

$\boldsymbol{\varphi X A N E S ~ I m a g i n g . ~ T h e ~ b e a m ~ e n e r g y ~ w a s ~ s e l e c t e d ~ u s i n g ~ a ~} \mathrm{Si}(311)$ double-crystal monochromator with a resolution of $\sim 0.5 \mathrm{eV} . \varphi$ XANES imaging was achieved by recording Fe XRF at 106 incident energies spanning the Fe K-edge $(7112 \mathrm{eV})$. Measurement energy interval was commensurate with anticipated structure in the XANES:

$\begin{array}{lllrll}7000 \mathrm{eV} \text { to } 7100 \mathrm{eV}: & 5 & \times & 20.0 \mathrm{eV} & \text { steps } \\ 7100 \mathrm{eV} \text { to } 7105 \mathrm{eV}: & 5 & \times & 1.0 \mathrm{eV} & \text { steps } \\ 7105 \mathrm{eV} \text { to } 7135 \mathrm{eV}: & 75 & \times & 0.4 \mathrm{eV} & \text { steps } \\ 7135 \mathrm{eV} \text { to } 7165 \mathrm{eV}: & 15 & \times & 2.0 \mathrm{eV} & \text { steps } \\ 7165 \mathrm{eV} \text { to } 7405 \mathrm{eV}: & 1 & \times & 240.0 \mathrm{eV} & \text { steps } \\ 7405 \mathrm{eV} \text { to } 7455 \mathrm{eV}: & 5 & \times & 5.0 \mathrm{eV} & \text { steps }\end{array}$

As for XFM, $\varphi$ XANES measurements used a beam spot $\sim 2 \times 2 \mu \mathrm{m}$ but data was recorded using continuous scanning at $0.2 \mathrm{~mm} \mathrm{sec}^{-1}$ (binned at $2 \mu \mathrm{m}$ intervals). Transit time through each virtual pixel

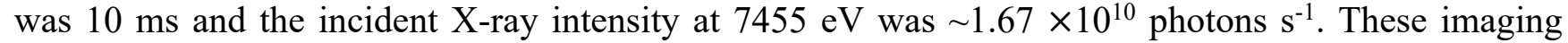
parameters gave a total dose associated with the $\varphi$ XANES measurement estimated at $\sim 5 \mathrm{MGy}$. This value is commensurate with doses typically delivered during bulk X-ray absorption spectroscopy.

$\boldsymbol{\varphi}$ XANES analysis. The XANES spectra from an iron foil $\left(50.1 \mu \mathrm{g} \mathrm{cm}{ }^{-2}\right.$, Micromatter Canada) was measured to monitor the energy calibration of the beamline. The maxima of the first peak in the derivative spectra of the iron foil was subsequently defined as $7112.0 \mathrm{eV}$. The energy stability of beamline has been determined at $<0.25 \mathrm{eV}$ over $24 \mathrm{hrs}$ making energy drift over the course of a scan negligible. Consistency of the measured edge positions in conjunction with stability of beam position and flux recorded in ion chambers upstream the specimen position provide confidence that energy stability was high through the duration of the experiment. Small position drifts were aligned by cross-correlation of the calcium map which remains essentially constant throughout the energy series.

XANES probes the density of states on the absorbing atom and reveals electronic and structural details of coordination environment. The aligned $\varphi$ XANES image series is stack of images, one per incident energy allowing the XANES of individual cells to be assessed. Previous work has shown that the distribution of calcium is a useful marker for the position of $C$. elegans intestinal cells and we used this information to identify regions of interest in the $\varphi$ XANES stack corresponding to anterior intestinal cells. Anterior intestinal cells were chosen due to their consistent and robust iron content ${ }^{51}$; see Figure $\mathbf{S 2}$ for the ROIs and iron maps used for the $\varphi$ XANES analysis.

As all points on the specimen represent a heterogenous mixture of iron binding species the resulting XANES spectra are admixtures with contributions from all of these components. The technical particulars of the XFM beamline (being primarily designed for elemental mapping) are not optimised for high resolution spectroscopy and our XANES spectra are relatively sparse. For iron K-edge XANES the abrupt increase in absorption coefficient at the critical threshold obscures the presence of $1 \mathrm{~s} \rightarrow 4 \mathrm{~s}$ and $1 \mathrm{~s} \rightarrow 4 \mathrm{p}$ electronic transitions. Berry et al ${ }^{59}$ demonstrated that the relative intensity of these transitions provides the proportional contribution of $\mathrm{Fe}^{2+}$ and $\mathrm{Fe}^{3+}$ to the XANES and can be assessed by interrogating the first derivative of the XANES spectra.

Lifespan determination. Lifespan was measured using established protocols ${ }^{2,60}$. SIH was dissolved in neat dimethyl sulfoxide (DMSO; Sigma-Aldrich) then added to the molten NGM at $55^{\circ} \mathrm{C}$ (to a final concentration of $250 \mu \mathrm{M} \mathrm{SIH}$ in $0.5 \% \mathrm{v} / \mathrm{v}$ DMSO). Lip-1 was dissolved in neat DMSO then added to the molten NGM at $55{ }^{\circ} \mathrm{C}$ (to a final concentration of $200 \mu \mathrm{M} \mathrm{Lip}-1$ in $0.5 \% \mathrm{v} / \mathrm{v}$ DMSO). Media containing equivalent vehicle alone $(0.5 \% \mathrm{v} / \mathrm{v}$ DMSO) was used for comparison. Standard overnight culture of the Escherichia coli (E. coli) strain OP50 was used as the food source.

Lifespan data was collected at $25( \pm 1){ }^{\circ} \mathrm{C}$ using the temperature sensitive-sterile strain TJ1060 [spe9(hc88); fer-15(b26)]. A synchronous population was obtained by transferring egg-laying adults to fresh plates at $16{ }^{\circ} \mathrm{C}$ for $2-3$ hours. The adults were removed and the plates with eggs then transferred to $25^{\circ} \mathrm{C}$ 
to ensure sterility. After 48 hours at $25{ }^{\circ} \mathrm{C}$, when worms were at the late L4/young adult stage, 25-35 nematodes were transferred to fresh plates containing either vehicle control, $250 \mu \mathrm{M} \mathrm{SIH}$, or $200 \mu \mathrm{M}$ Lip-1. All plates were coded to allowing blinding of the experimenter to the treatment regime during scoring. Nematodes were scored for survival at one to three-day intervals and transferred to freshly prepared plates as needed (2-5 days).

C. elegans are bacteriophores and the E. coli (OP50) monoxenic diet can colonize the pharynx and intestine, resulting in death. Consequently, antibiotics are known to extend C. elegans lifespan ${ }^{61}$. In addition, iron chelating compounds, such as EDTA have been reported to have antibiotic properties. We performed a disk diffusion test ${ }^{62}$ on both Lip-1 and SIH and observed no evidence for inhibition of $E$. coli (strain OP50) growth). Furthermore, an additive effect on median lifespan extension was seen when $\mathrm{SIH}$ and the antibiotic ampicillin were co-administered to C. elegans (Supplemental Figure S15), consistent with independent effects on lifespan.

It is well documented that differences are observed between independent measures of lifespan, with micro-environmental factors such as minor temperature fluctuations potentially resulting differences in median and maximum lifespan between replicates ${ }^{63}$. After determining the optimal doses of $250 \mu \mathrm{M}$ SIH and $200 \mu \mathrm{M}$ Lip-1, respectively, cohorts of nematodes were compared in 8 independent replicates. As the number of worms measured is known to influence the likelihood of accurately observing differences in lifespan ${ }^{64}$, the starting populations for all treatments within experiments were in excess of 70 individuals. The median and maximum lifespans observed of control and treated populations for these 8 replicates are shown in Table S8. As can be seen in this table, the median lifespan of treated populations was always greater than that of control populations, however the magnitude of the difference varied between experiments, with the median lifespan of control populations ranging from 7 to 9 days.

Body size analysis. A developmentally synchronous population, derived from eggs laid over a 2-hour window, were cultured on NGA media at $25^{\circ} \mathrm{C}$ for $48 \mathrm{~h}$, and then as young adult worms were transferred onto three treatment plates for an additional $24 \mathrm{~h}$. The treatment plates included NGA with $0.5 \%(\mathrm{v} / \mathrm{v})$ DMSO (vehicle control, Ctl), $250 \mu \mathrm{M} \mathrm{SIH}$, or $200 \mu \mathrm{M}$ Lip-1 (as described above).

Cohorts of approximately 100 animals were transferred into a $1.5 \mathrm{ml}$ centrifuge tube containing $400 \mu \mathrm{L}$ S-basal. Following a brief centrifugation excess S-basal was removed leaving the animals suspended in $50 \mu \mathrm{L}$. Animals were euthanised and straightened by a 15 second exposure to $60{ }^{\circ} \mathrm{C}$ (using a heated water bath). Samples were then mounted between glass slides and a cover slip and immediately imaged. Micrographs were collected using a Leica M80 stereomicroscope and Leica DFC290 HD 3 MP) digital camera. Pixel sizes were defined using a calibrated $25 \mu \mathrm{m}$ grid slide (Microbrightfield, Inc). Size and shape metrics were extracted from brightfield images were analysed using the WormSizer plugin ${ }^{65}$ for ImageJ.

Fertility analysis. Wild type (N2) adults (4-day post egg lay) were transferred to fresh plates for 30 minutes at $20{ }^{\circ} \mathrm{C}$ to establish a developmentally synchronous population. Adult nematodes were then removed, and eggs were then transferred to $25^{\circ} \mathrm{C}$. As with the survival analyses, after 48 hours at $25^{\circ} \mathrm{C}$, when worms were at the late L4/young adult stage individual nematodes were transferred to plates containing vehicle control, $250 \mu \mathrm{M}$ SIH, or $200 \mu \mathrm{M}$ Lip-1. After 24 hours, adult worms were transferred to fresh plates and transferred daily until the end of the fertile period. After allowing progeny to develop for 2 days at $20^{\circ} \mathrm{C}$, they were then counted to determine daily and total fertility. Early fertility is determined by the number of progeny laid in the first 24-hour period.

Movement. A developmentally synchronous population, derived from eggs laid over a 2-hour window, were cultured on NGA media at $25^{\circ} \mathrm{C}$ for $48 \mathrm{~h}$, and then as young adult worms were transferred onto three treatment plates for an additional $24 \mathrm{~h}$. The treatment plates included NGM $+0.5 \%(\mathrm{v} / \mathrm{v}) \mathrm{DMSO}$ (vehicle control, Ctl), NGM + $250 \mu \mathrm{M} \mathrm{SIH}$, and NGM + $200 \mu \mathrm{M}$ Lip-1 (as described above).

Single worms were transferred to a $55 \mathrm{~mm}$ NGA assay plate devoid of a bacterial lawn, without a lid, and left to recover from the transfer for 2 minutes. Movement of the adults was then recorded using a stereomicroscope (Leica M80) with transmitted illumination from below. A 30 second video recording was captured using a 3 MP DFC290 HD digital camera (Leica Microsystems) at a rate of 30 frames per second. Pixel length was calibrated using a $25 \mu \mathrm{m}$ grid slide (Microbrightfield, Inc). Recorded series 
were analysed using the wrMTrck plugin ${ }^{66}$ for ImageJ (www.phage.dk/plugins) and Fiji ${ }^{67}$ (a distribution of ImageJ).

The maximum velocity achieved was expressed as mm per second (as derived from the distance between displaced centroids per second). Additional metrics of movement were determined including mean velocity $\left(\mathrm{mm} \mathrm{s}^{-1}\right)$ and (total) distance travelled $(\mathrm{mm})$. These variables were collated in Prism (v7.0a GraphPad Software) and presented as a scatter plot with medians and interquartile range.

\section{Quantification and Statistical Analysis}

Areal density and total iron analysis. Areal iron and total body iron data were assessed for normality using a D'Agostino \& Pearson test (see Supplemental Tables S3 and S5). Based on this analysis a oneway ANOVA was performed followed by a Sidak's multiple comparisons test (as implemented by PRISM; see Supplemental Tables S4 and S6).

Standard lifespan analysis. Kaplan-Meier survival curves were generated and compared via nonparametric log rank tests (Prism v7.0a, GraphPad Software).

Testing for Departure from Temporal Rescaling. Following the recently published results of Stroustrup et al. we determined whether the results observed with both the SIH and Lip-1 interventions were due to temporal scaling of ageing. A modified Kolmogorov-Smirnov (K-S) test was applied to the residuals from a replicate-specific accelerated failure time (AFT) model fitted according to the Buckley-James method that uses a nonparametric baseline hazard function. The function $b j$ in $\mathrm{R}$ package $r m s$ was used to fit the replicate-specific model with interventions as categorical independent variables. We used the same approach for testing whether the temperature difference results in simple temporal rescaling, with the only difference being using temperature rather than intervention as categorical independent variable in the AFT model. Full details of these analyses are included in the online Supplementary information.

Characterizing Departure from Temporal Rescaling. Parametric survival models with Weibull baseline hazards and Gamma frailty were fitted to replicate-specific data using the R package flexsurv. A likelihood ratio test was used to compare models that assume simple temporal rescaling to models that allow varying degrees of departure from temporal rescaling. The best model for each replicate was selected using a likelihood ratio test and the goodness of fit (GOF) of the best model is evaluated using a chi-square GOF test. To combine data across different replicates, we performed fixed-effect and random-effect meta-analyses for each parameter in the best model (Supplemental Table S13). Briefly, the fixed-effect meta-analysis estimates were derived using Inverse Variance Weighting (IVW) in which the estimates from each replicate were weighted by the inverse of their variance estimates. The metaanalysis estimates were then calculated simply as the weighted average of estimates from all replicates. The fixed-effect meta-analysis assumes that there is insignificant variation between the estimates of the same parameter across different replicates. The random-effect meta-analysis also derives the estimates by assigning weights to estimates from each replicate, but in this case the weights take into account the variation of estimates across replicates.

The fixed-effects and random-effects meta-analysis estimates are quite similar and in Supplemental Figure S9 we can see that the meta-analysis estimates provide the best fit to SIH data and worst for Lip1 data. Since there is significant between-replicate variation for the majority of the parameters, it is not surprising that the when the meta-analysis estimates are applied to the real data, a chi-square goodness of fit reveals significant lack of fit $\left(\chi^{2}{ }_{(3)}=237.0\right.$ for control worms, $\chi^{2}{ }_{(5)}=258.0$ for Lip-1 and $\chi^{2}{ }_{(3)}=$ 49.7 for $\mathrm{SIH}$, all p-values $<0.001)$.

One notable pattern from Table S13 is that for nearly all replicates, there is more heterogeneity due to unobserved factors among the control worms, as indicated by the negative $\Delta \log \left(\sigma^{2}\right)$ parameter estimates for Lip-1 and SIH data. This heterogeneity is also reflected in a de-acceleration of the hazard function for control worms (Supplemental Figures S9c-d) beyond 7-8 days. This de-acceleration of the hazard function is the main contributor to the crossing behaviour we observe when comparing the survival functions (Supplemental Figure S10), and it is what causes a violation of the simple temporal rescaling assumption.

Survival during GSH depletion. For survival with increasing DEM dose response and protection by compounds (Lip-1 and SIH), data was plotted as fraction of animal alive with upper and lower 95\% 
confidence interval, using the Wilson 'score' method ${ }^{68}$ using asymptotic variance ${ }^{46}$ and fitted with a sigmoidal curve (Prism). Pairwise comparisons of treated groups versus control at each concentration of DEM was determined using the $\mathrm{N}-1$ chi-squared test ${ }^{69,70}$.

Fertility. Differences in fertility (i.e. early and total reproductive output) were assessed using an ordinary one-way analysis of variance (ANOVA), followed by a Tukey's multiple comparison test (as implemented by Prism v7.0a, GraphPad Software).

Body length and volume analysis. Data of estimated adult body length and volume were initially assessed for normality using a D'Agostino \& Pearson test (see Supplemental Tables S18 and S20). Based on this analysis a nonparametric Kruskal-Wallis Analysis of Variance (ANOVA) was performed followed by a Dunn-Šidák test for multiple comparisons (as implemented by Prism v7.0a, GraphPad Software; Supplemental Tables S19 and S21).

Movement Analysis. Data of estimated maximum velocity were initially assessed for normality (see Supplemental Table S22). Based on this analysis a nonparametric Kruskal-Wallis ANOVA was performed followed by a Dunn-Šidák test for multiple comparisons (as implemented by PRISM; Supplemental Table S23). Mean velocity and total distance travelled were also determined (Supplemental Figures S12-13). Results summaries and comparisons between treatments are shown in Supplemental Tables S24-27. The data for the three movement parameters were combined across treatments and ages to determine the relationship between the estimated parameters, all were found to be positively correlated (Supplemental Figure S14).

Cell death analysis. Differences between the proportion of live animals with fluorescently labelled nuclei in control versus Lip-1 and SIH treatment, either aged or exposed to DEM, were compared using a z-test.

Type I error for statistical hypothesis testing. Unless otherwise stated, all statistical tests are conducted with type I error set at 0.05 . 


\section{SUPPLEMENTARY INFORMATION}

\section{Glutathione determination}

\section{Glutathione and aging}

There was a significant reduction in glutathione levels with increased adult age in C. elegans (Figure 1c). For comparisons between age and treatment groups an Ordinary one-way ANOVA was performed, followed by Tukey's multiple comparisons test (ANOVA: $F(3,20)=32.96, p<0.0001$ ). The results of the pairwise comparisons, corrected for multiple comparisons, are shown in Table $\mathbf{S 1 .}$

Table S1: Summary of glutathione level comparisons between ages

\begin{tabular}{|l|r|r|r|r|}
\hline $\begin{array}{l}\text { Tukey's multiple } \\
\text { comparisons test }\end{array}$ & \multicolumn{1}{|c|}{ Mean Diff. } & 95.00\% CI of diff. & Significant? & \multicolumn{1}{l|}{$\begin{array}{l}\text { Adjusted } p \\
\text { Value }\end{array}$} \\
\hline Day 1 vs. Day 4 & 0.1964 & 0.08439 to 0.3085 & Yes & 0.0003 \\
\hline Day1 vs. Day 8 & 0.2738 & 0.1618 to 0.3858 & Yes & $<0.0001$ \\
\hline Day 1 vs. Day 10 & 0.3667 & 0.2546 to 0.4787 & Yes & $<0.0001$ \\
\hline Day 4 vs. Day 8 & 0.07738 & -0.03466 to 0.1894 & No & 0.2992 \\
\hline Day 4 vs. Day 10 & 0.1702 & 0.0582 to 0.2823 & Yes & 0.0015 \\
\hline Day 8 vs. Day 10 & 0.09286 & -0.01918 to 0.2049 & No & 0.1423 \\
\hline
\end{tabular}

\section{Glutathione depletion}

There was a significant reduction in glutathione levels after treatment with of 4 Day old adults with DEM (Figure 1f). Pre-treatment with SIH protected against this reduction, with this treatment also resulting in a higher basal level of glutathione. For comparisons between treatment groups an Ordinary one-way ANOVA was performed, followed by Tukey's multiple comparisons test (ANOVA: F $(5,30)$ $=50.97, p<0.0001)$. The results of the pairwise comparisons, corrected for multiple comparisons, are shown in Table S2.

Table S2: Summary of glutathione level comparisons after DEM exposure with pre-treatment

\begin{tabular}{|l|c|c|c|r|}
\hline Tukey's multiple comparisons test & Mean Diff. & 95 \% CI of diff. & $\begin{array}{c}\text { Significant } \\
?\end{array}$ & $\begin{array}{r}\text { Adjusted } \\
p \text { Value }\end{array}$ \\
\hline Day 4 Control vs Day 4 Lip-1 & -0.04508 & -0.3588 to 0.2686 & No & 0.9997 \\
\hline Day 4 Control vs Day 4 SIH & -0.6447 & -0.9584 to -0.331 & Yes & $<0.0001$ \\
\hline Day 4 Ctl vs. Day 4 Ctl + DEM & 0.6921 & 0.409 to 0.9752 & Yes & $<0.0001$ \\
\hline Day 4 Lip-1 vs. Day 4 Lip-1 + DEM & 0.7379 & 0.4515 to 1.024 & Yes & $<0.0001$ \\
\hline Day 4 SIH vs. Day 4 SIH + DEM & 0.7667 & 0.4857 to 1.048 & Yes & $<0.0001$ \\
\hline Day 4 Ctl + DEM vs. Day 4 Lip-1 + DEM & 0.0006989 & -0.2518 to 0.2532 & No & $>0.9999$ \\
\hline Day 4 Ctl + DEM vs. Day 4 Lip-1 + DEM & -0.5701 & -0.8166 to -0.3237 & Yes & $<0.0001$ \\
\hline
\end{tabular}




\section{Supplemental Analysis of X-ray Fluorescence Mapping}

Figure S1: The following figures show the ROIs associated with each iron map (ROI; white = overlapped/fractional, green $=$ whole/minimally overlapped, red $=$ excluded from analysis). Shown are the masks used to identify and analyse the iron elemental maps of TJ1060 populations at different adult ages \pm Lip- 1 or $\mathrm{SIH}$ at $25^{\circ} \mathrm{C}$.

Figure S1A: Day 1 adults (i.e. first day of adulthood)

Figure S1B: Day 4 Control

Figure S1C: Day $4+$ Lip-1

Figure S1D: Day $4+\mathrm{SIH}$

Figure S1E: Day 8 Control

Figure S1F: Day $8+$ Lip-1

Figure S1G: Day 8 SIH
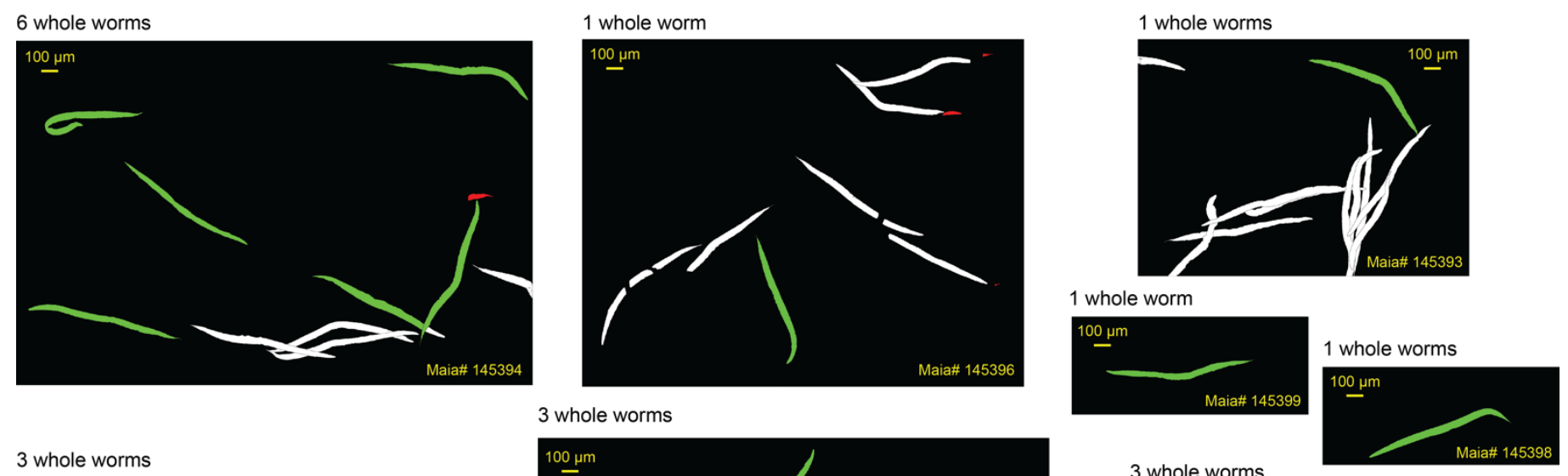

3 whole worms

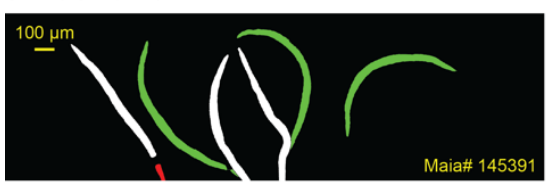

3 whole worms
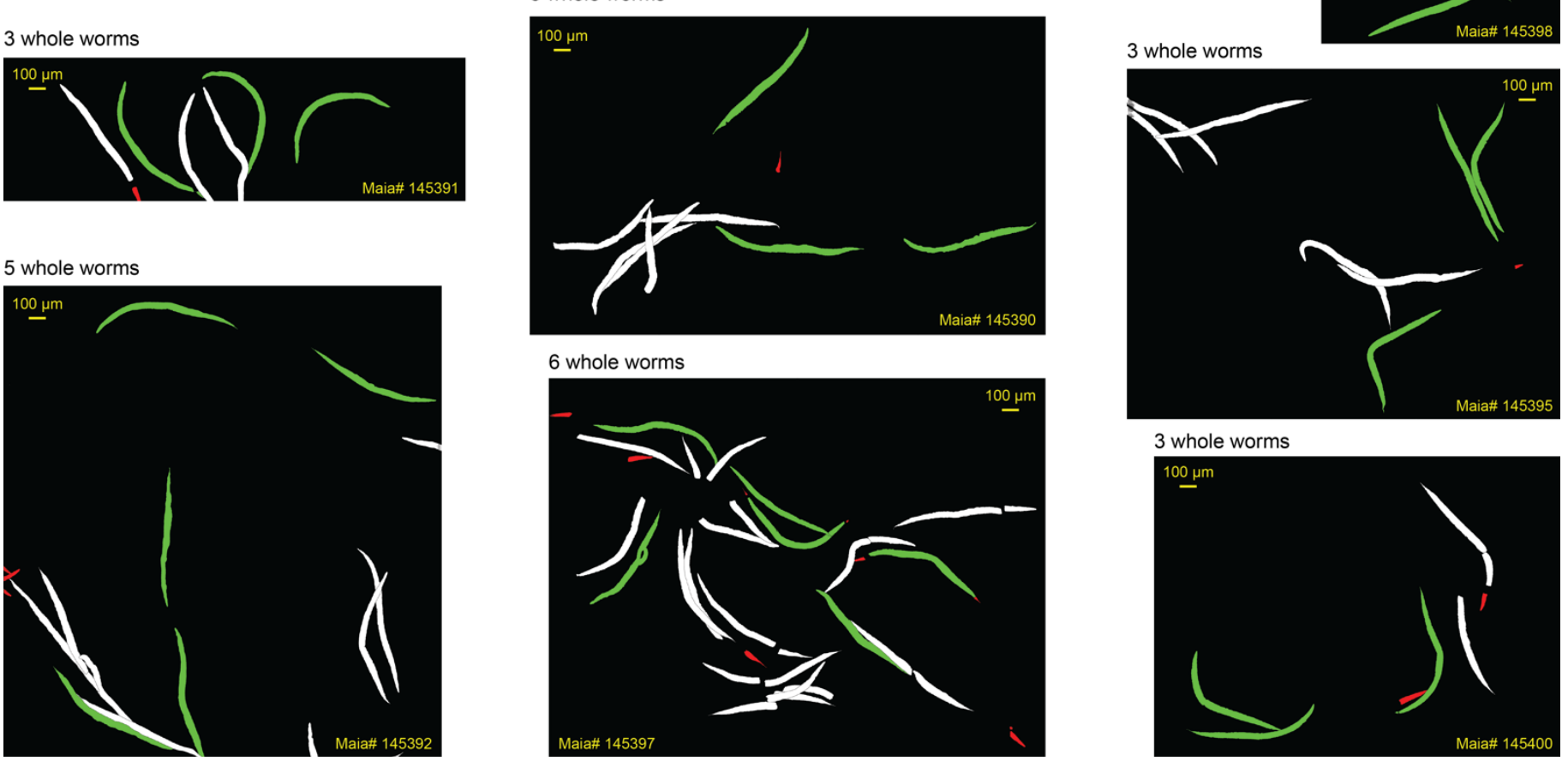

3 whole worms

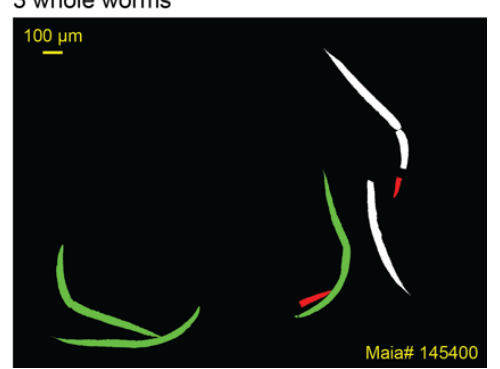

Figure S1A. Masks for 1 day old adults (starting population) 
11 whole worms

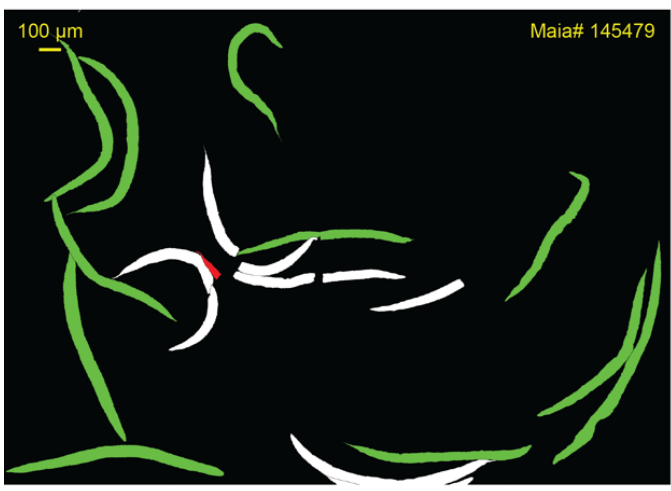

1 whole worm

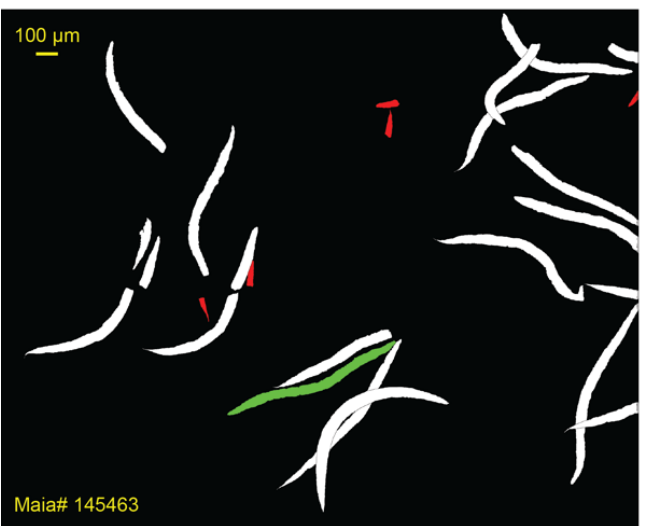

6 whole worms

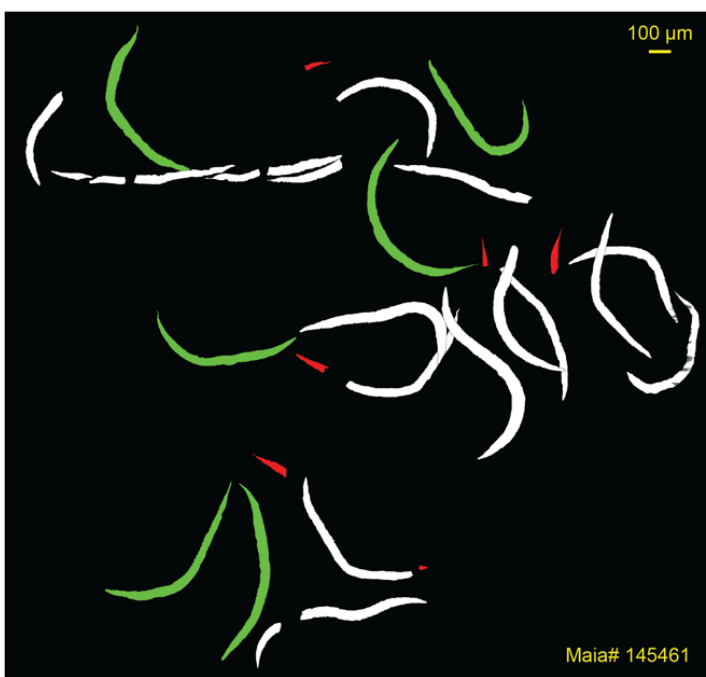

2 whole worm

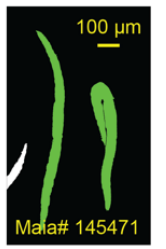

1 whole worm

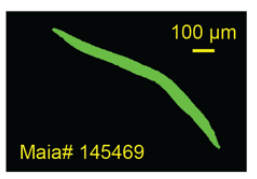

Figure S1B. Masks for 4 day old Control adults

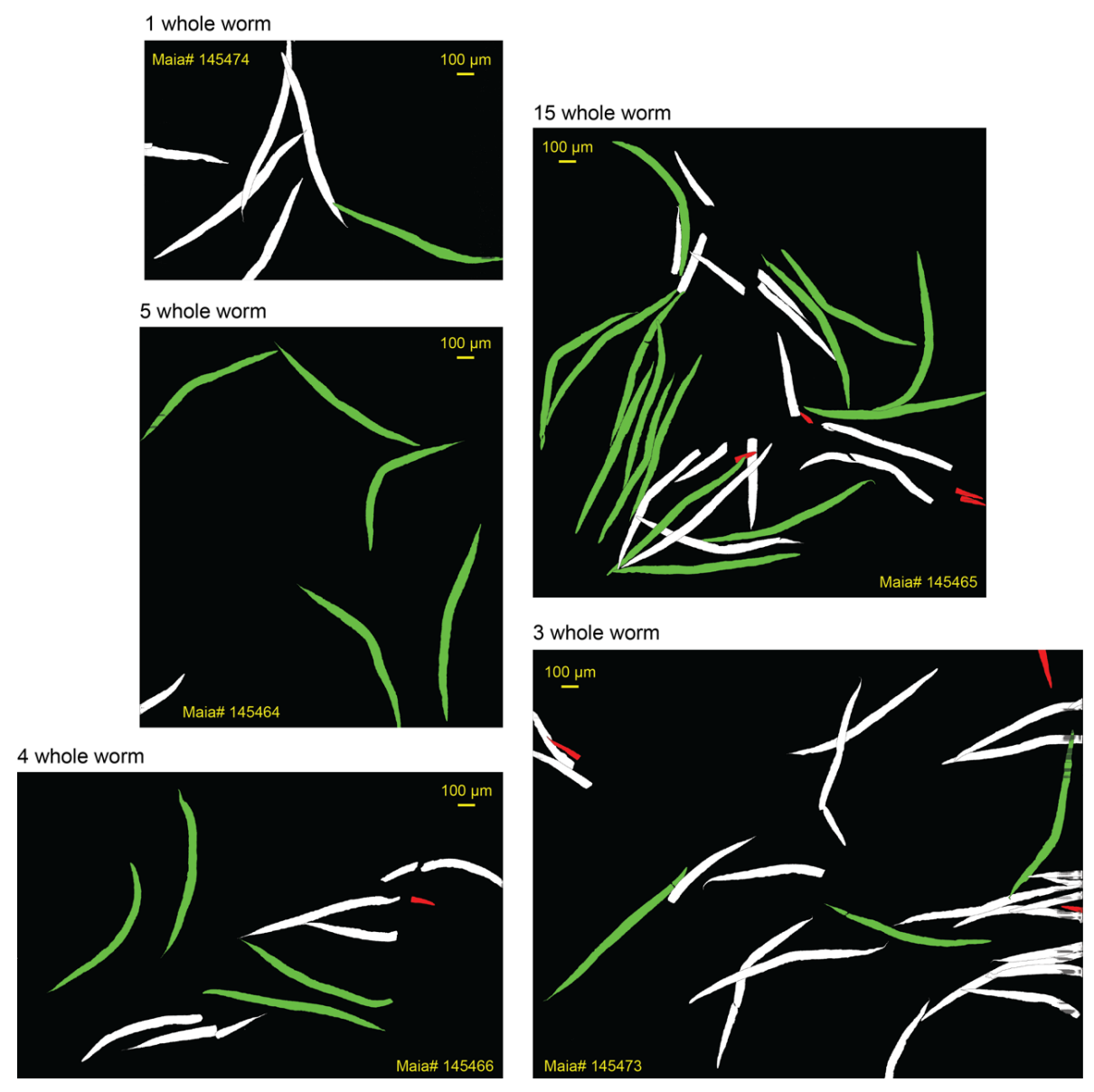

Figure S1C. Masks for 4 day old SIH treated adults 


\section{0 whole worms}

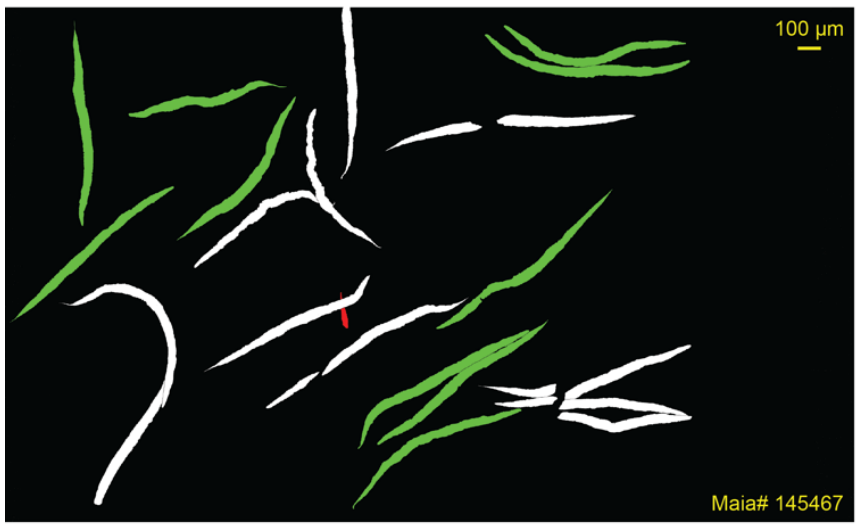

2 whole worms

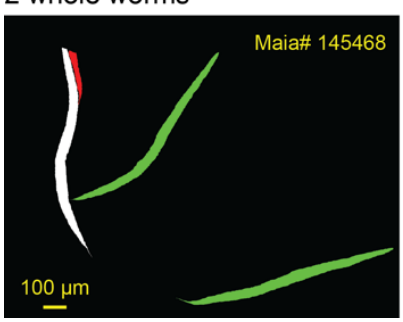

0 whole worms
7 whole worms

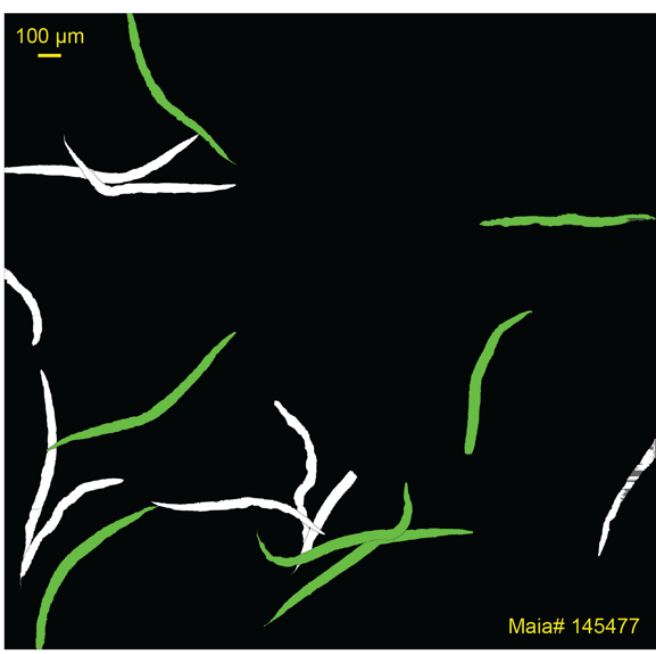

0 whole worms

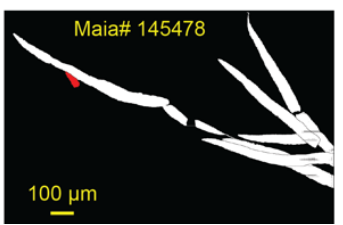

1 whole worm

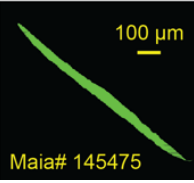

Figure S1D. Masks for 4 day old Lip-1 treated adults

5 whole worms

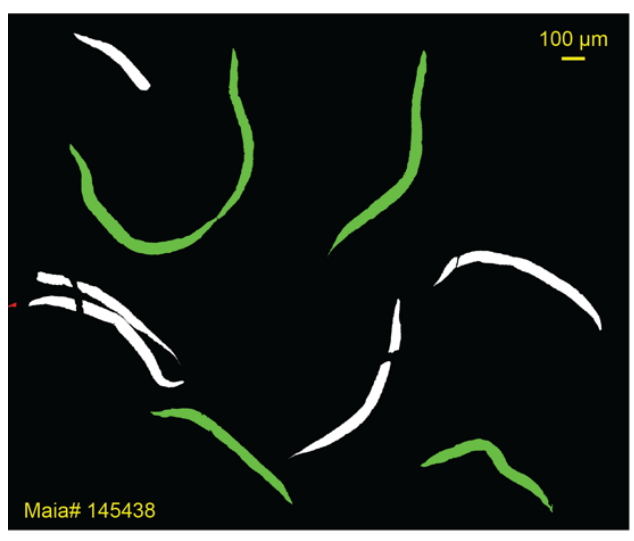

1 whole worm

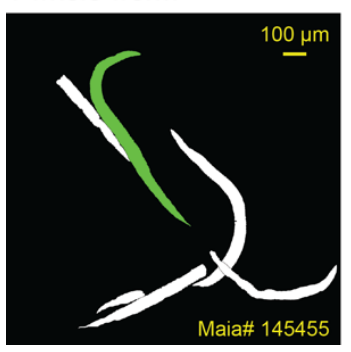

1 whole worm

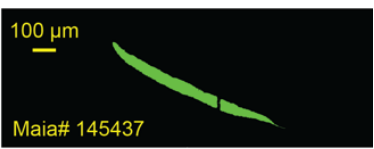

2 whole worms

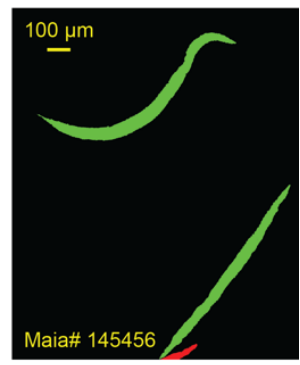

Figure S1E. Masks for 8 day old Control adults

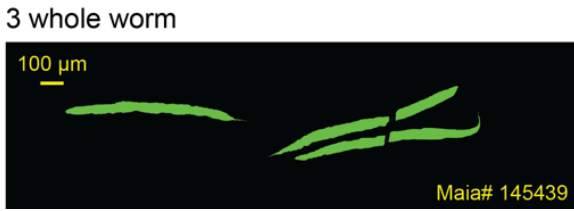



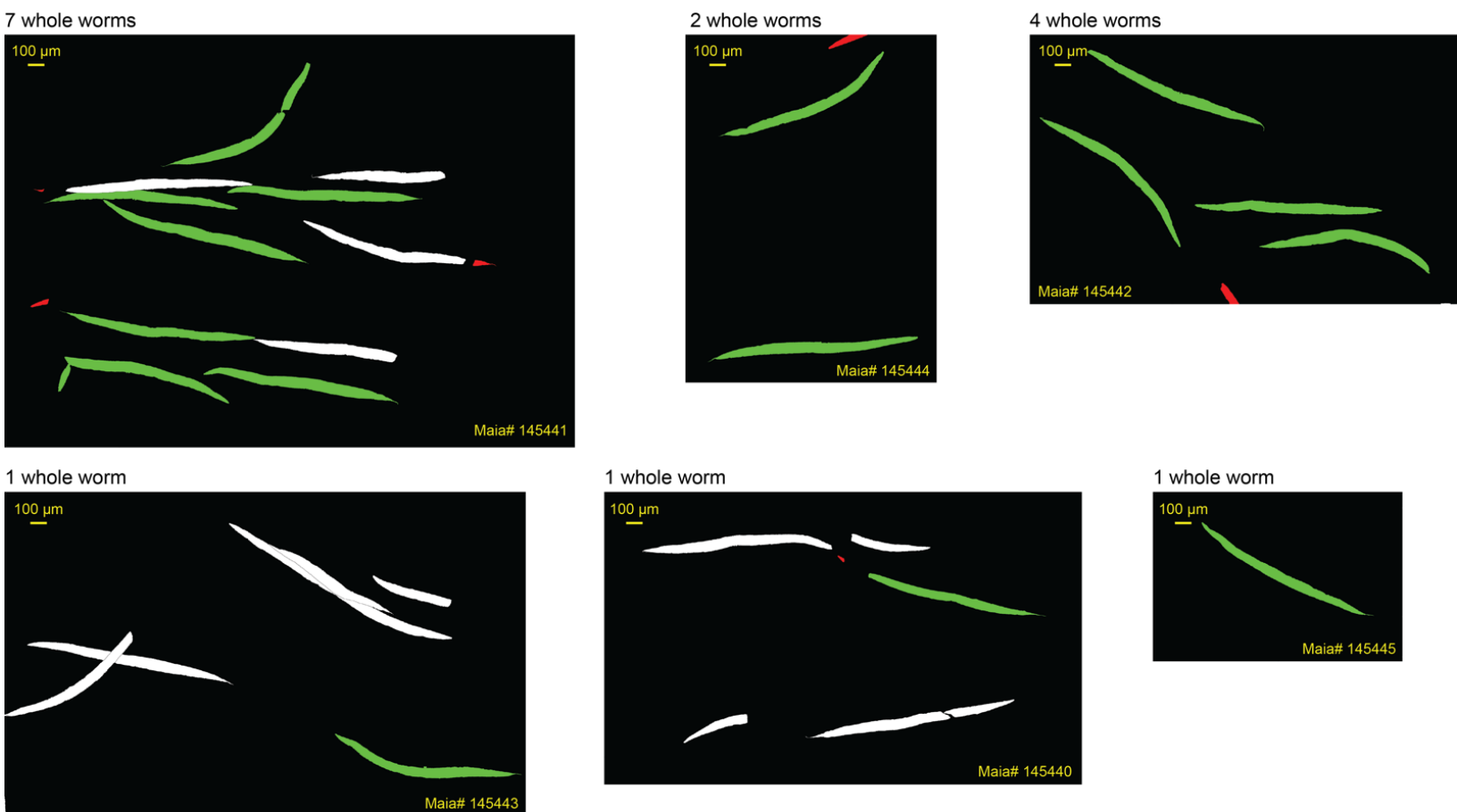

Figure S1F. Masks for 8 day old $250 \mu \mathrm{M}$ SIH treated adults
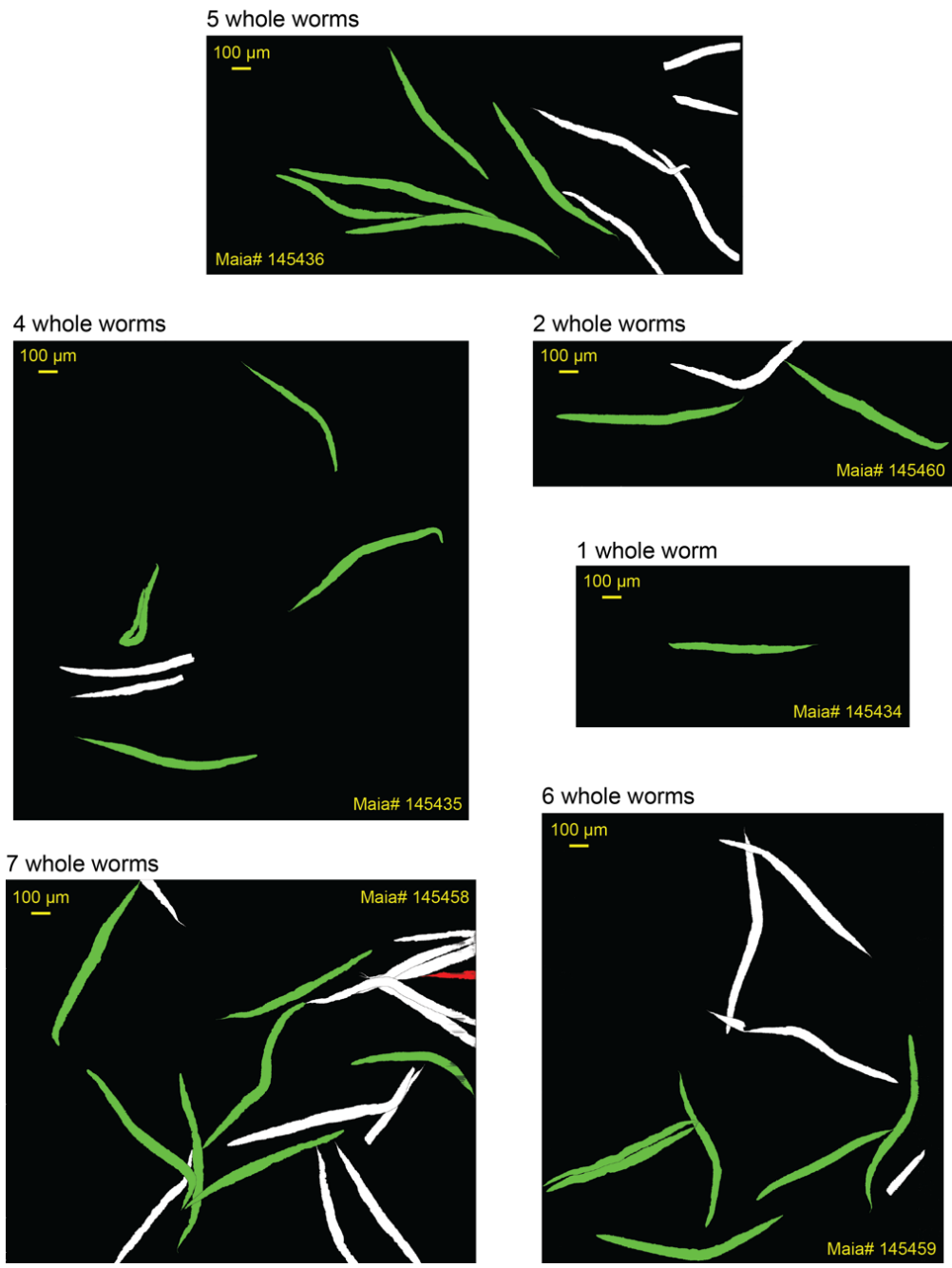

Figure S1G. Masks for 8 day old $200 \mu$ M Lip-1 treated adults 


\section{Analysis of Iron from X-ray Fluorescence Microscopy}

\section{Total Mean areal density of iron analysis}

Summary statistics and tests for normality of areal density for iron $\left(\mathrm{pg} \mu \mathrm{m}^{-2}\right)$ are included in Table S3. All the total iron data sets were normally distributed, as indicated below.

Table S3: Summary of areal density iron results between treatments and ages

\begin{tabular}{|c|c|c|c|c|c|c|c|}
\hline & Day 1 & $\begin{array}{c}\text { Day } 4 \\
\text { Control }\end{array}$ & $\begin{array}{c}\text { Day } 4 \\
\text { SIH }\end{array}$ & $\begin{array}{l}\text { Day } 4 \\
\text { Lip-1 }\end{array}$ & $\begin{array}{l}\text { Day } 8 \\
\text { Control }\end{array}$ & $\begin{array}{c}\text { Day } 8 \\
\text { SIH }\end{array}$ & $\begin{array}{l}\text { Day } 8 \\
\text { Lip-1 }\end{array}$ \\
\hline Number of values & 32 & 25 & 27 & 20 & 12 & 17 & 22 \\
\hline Minimum & 177.7 & 443.4 & 267.1 & 590.5 & 629.6 & 324.2 & 644 \\
\hline $25 \%$ Percentile & 216.2 & 592.2 & 298.3 & 630.3 & 677.6 & 381.9 & 726.5 \\
\hline Median & 236 & 663.6 & 304.4 & 711.4 & 823.8 & 408.9 & 800.5 \\
\hline $75 \%$ Percentile & 254.9 & 739.1 & 357.3 & 732.2 & 885.3 & 501.9 & 892.4 \\
\hline Maximum & 271.7 & 894 & 412.1 & 851.8 & 1100 & 558 & 1143 \\
\hline Mean & 234 & 666.2 & 321.8 & 698.5 & 820.3 & 434.7 & 821.6 \\
\hline Std. Deviation & 26.61 & 111.3 & 37.49 & 67.26 & 145.1 & 74.49 & 137.4 \\
\hline \begin{tabular}{|l|} 
Std. Error of \\
Mean
\end{tabular} & 4.704 & 22.26 & 7.216 & 15.04 & 41.87 & 18.07 & 29.29 \\
\hline $\begin{array}{l}\text { Lower 95\% CI of } \\
\text { mean }\end{array}$ & 224.4 & 620.3 & 307 & 667 & 728.2 & 396.4 & 760.7 \\
\hline $\begin{array}{l}\text { Upper } 95 \% \text { CI of } \\
\text { mean }\end{array}$ & 243.6 & 712.1 & 336.7 & 730 & 912.5 & 473 & 882.5 \\
\hline \multicolumn{8}{|c|}{ D'Agostino \& Pearson normality test } \\
\hline $\mathrm{K} 2$ & 2.672 & 0.2089 & 3.471 & 0.4574 & 0.6834 & 1.74 & 5.565 \\
\hline$p$ value & 0.2629 & 0.9008 & 0.1763 & 0.7956 & 0.7105 & 0.4190 & 0.0619 \\
\hline $\begin{array}{l}\text { Passed normality } \\
\text { test }(\alpha=0.05) ?\end{array}$ & Yes & Yes & Yes & Yes & Yes & Yes & Yes \\
\hline$p$ value summary & ns & ns & ns & ns & ns & ns & ns \\
\hline
\end{tabular}

There was a significant difference between mean areal density of iron $(\mathrm{F}(6,148)=171.3, p<0.0001)$ amongst the groups measured. Comparisons between age and treatment groups an Ordinary one-way ANOVA was performed, followed by Sidak's multiple comparisons test. The results of the pairwise comparisons, corrected for multiple comparisons, are shown in Table S4.

Table S4: Summary of areal density of iron comparisons between ages and treatments

\begin{tabular}{|l|r|r|c|r|}
\hline Sidak's multiple comparisons test & $\begin{array}{c}\text { Mean } \\
\text { Diff. }\end{array}$ & $95.00 \%$ CI of diff. & Significant? & $\begin{array}{c}\text { Adjusted } p \\
\text { value }\end{array}$ \\
\hline Day 1 Control vs. Day 4 Control & -432.2 & -499.3 to -365.1 & Yes & $<0.0001$ \\
\hline Day 1 Control vs. Day 8 Control & -586.3 & -671.5 to -501.2 & Yes & $<0.0001$ \\
\hline Day 4 Control vs. Day 8 Control & -154.1 & -242.4 to -65.85 & Yes & $<0.0001$ \\
\hline Day 4 Control vs. Day 4 SIH & 344.4 & 274.6 to 414.1 & Yes & $<0.0001$ \\
\hline Day 4 Control vs. Day 4 Lip-1 & -32.28 & -107.7 to 43.15 & No & 0.9226 \\
\hline Day 8 Control vs. Day 8 SIH & 385.6 & 290.9 to 480.4 & Yes & $<0.0001$ \\
\hline Day 8 Control vs. Day 8 Lip-1 & -1.229 & -91.45 to 89 & No & $>0.9999$ \\
\hline Day 4 SIH vs. Day 8 SIH & -112.9 & -190.7 to -35.02 & Yes & 0.0006 \\
\hline Day 4 Lip-1 vs. Day 8 Lip-1 & -123.1 & -200.8 to -45.42 & Yes & 0.0001 \\
\hline Day 4 SIH vs. Day 4 Lip-1 & -376.6 & -450.8 to -302.5 & Yes & $<0.0001$ \\
\hline Day 8 SIH vs. Day 8 Lip-1 & -389.9 & -469 to -304.8 & Yes & $<0.0001$ \\
\hline
\end{tabular}




\section{Total body iron analysis}

Summary statistics and tests for normality of total body iron (pg) are included in Table S5. All the total iron data sets were normally distributed, as indicated below.

Table S5: Summary of total body iron results between treatments and ages

\begin{tabular}{|c|c|c|c|c|c|c|c|}
\hline & Day 1 & $\begin{array}{l}\text { Day } 4 \\
\text { Control }\end{array}$ & $\begin{array}{c}\text { Day } 4 \\
\text { SIH }\end{array}$ & $\begin{array}{l}\text { Day } 4 \\
\text { Lip-1 }\end{array}$ & $\begin{array}{l}\text { Day } 8 \\
\text { Control }\end{array}$ & $\begin{array}{c}\text { Day } 8 \\
\text { SIH }\end{array}$ & $\begin{array}{l}\text { Day } 8 \\
\text { Lip-1 }\end{array}$ \\
\hline Number of values & 32 & 25 & 27 & 20 & 12 & 17 & 22 \\
\hline Minimum & 13.48 & 91.73 & 41.07 & 71.51 & 122.8 & 74.16 & 100 \\
\hline $25 \%$ Percentile & 22.06 & 109.2 & 61.28 & 91.34 & 134.3 & 117.5 & 128.9 \\
\hline Median & 25.36 & 124.4 & 66.78 & 114.6 & 146.6 & 127.5 & 157.2 \\
\hline $75 \%$ Percentile & 27.75 & 138.6 & 71.8 & 129.5 & 173.2 & 169.5 & 189.6 \\
\hline Maximum & 39.6 & 171.1 & 98.92 & 148.6 & 214.2 & 202 & 277.9 \\
\hline Mean & 24.79 & 126.1 & 68.37 & 113.3 & 154.4 & 138.6 & 165.1 \\
\hline Std. Deviation & 5.166 & 21.05 & 12.1 & 21.91 & 27.94 & 36.58 & 44.93 \\
\hline Std. Error of Mean & 0.9133 & 4.211 & 2.328 & 4.9 & 8.065 & 8.873 & 9.579 \\
\hline Lower $95 \%$ CI of mean & 22.93 & 117.4 & 63.59 & 103.1 & 136.7 & 119.8 & 145.2 \\
\hline Upper $95 \%$ CI of mean & 26.65 & 134.8 & 73.16 & 123.6 & 172.2 & 157.4 & 185 \\
\hline \multicolumn{8}{|c|}{ D'Agostino \& Pearson normality test } \\
\hline $\mathrm{K} 2$ & 2.708 & 0.8963 & 4.252 & 1.75 & 3.123 & 0.6397 & 4.128 \\
\hline$p$ value & 0.2582 & 0.6388 & 0.1193 & 0.4169 & 0.2099 & 0.7262 & 0.1270 \\
\hline $\begin{array}{l}\text { Passed normality test } \\
(\alpha=0.05) ?\end{array}$ & Yes & Yes & Yes & Yes & Yes & Yes & Yes \\
\hline$p$ value summary & $\mathrm{ns}$ & ns & ns & ns & ns & ns & $\mathrm{ns}$ \\
\hline
\end{tabular}


There was a significant difference between total body iron $(\mathrm{F}(6,148)=97.3, p<0.0001)$ amongst the groups measured. Comparisons between age and treatment groups an Ordinary one-way ANOVA was performed, followed by Sidak's multiple comparisons test. The results of the pairwise comparisons, corrected for multiple comparisons, are shown in Table S6.

Table S6: Summary of total body iron between ages and treatments.

\begin{tabular}{|l|r|r|r|r|}
\hline Sidak's multiple comparisons test & $\begin{array}{c}\text { Mean } \\
\text { Diff. }\end{array}$ & $95.00 \%$ CI of diff. & Significant? & $\begin{array}{l}\text { Adjusted } \\
p \text { Value }\end{array}$ \\
\hline Day 1 Control vs. Day 4 Control & -101.3 & -120.9 to -81.67 & Yes & $<0.0001$ \\
\hline Day 1 Control vs. Day 8 Control & -129.6 & -154.5 to -104.8 & Yes & $<0.0001$ \\
\hline Day 4 Control vs. Day 8 Control & -28.35 & -54.16 to -2.538 & Yes & 0.0211 \\
\hline Day 4 Control vs. Day 4 SIH & 57.71 & 37.31 to 78.11 & Yes & $<0.0001$ \\
\hline Day 4 Control vs. Day 4 Lip-1 & 12.74 & -9.306 to 34.79 & No & 0.6818 \\
\hline Day 8 Control vs. Day 8 SIH & 15.8 & -11.91 to 43.51 & No & 0.6991 \\
\hline Day 8 Control vs. Day 8 Lip-1 & -10.7 & -37.07 to 15.68 & No & 0.9550 \\
\hline Day 4 SIH vs. Day 8 SIH & -70.26 & -93.01 to -47.5 & Yes & $<0.0001$ \\
\hline Day 4 Lip-1 vs. Day 8 Lip-1 & -51.79 & -74.49 to -29.08 & Yes & $<0.0001$ \\
\hline Day 4 SIH vs. Day 4 Lip-1 & -44.97 & -66.65 to -23.29 & Yes & $<0.0001$ \\
\hline Day 8 SIH vs. Day 8 Lip-1 & -26.49 & -50.23 to -2.763 & Yes & 0.0178 \\
\hline
\end{tabular}

\section{Analysis of Iron from X-ray Absorption Near Edge Spectroscopy ( $\varphi$-XANES)}

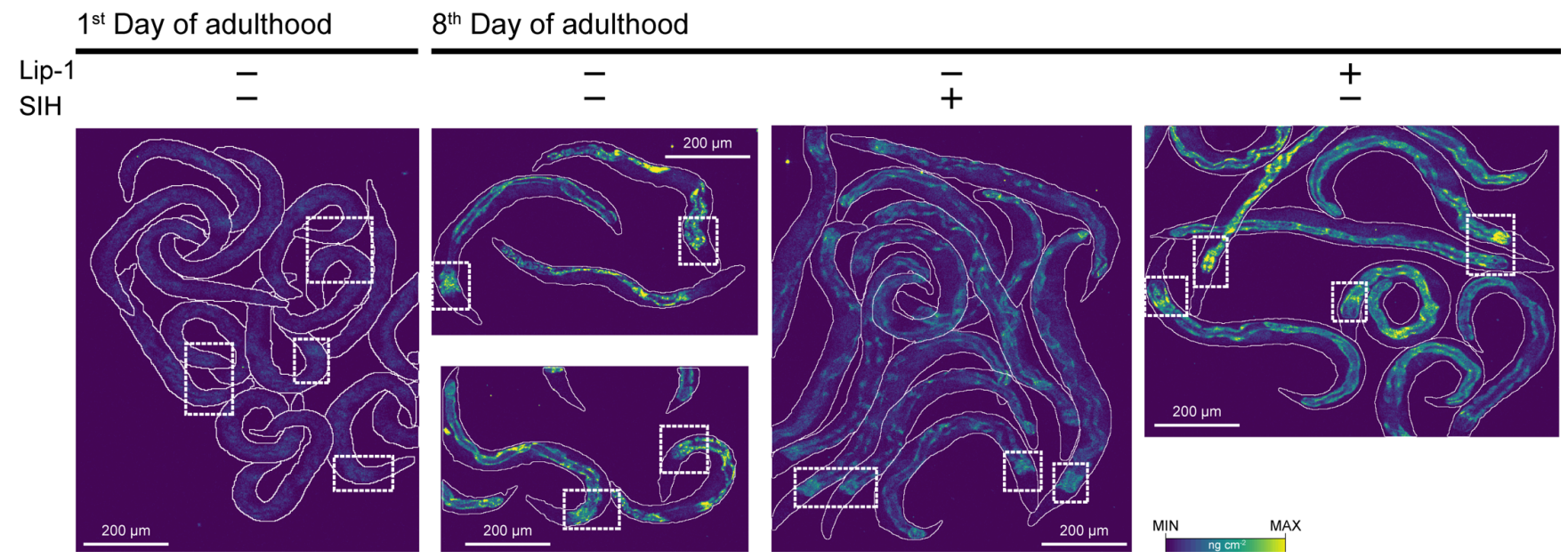

Figure S2: XFM maps of Fe and regions-of-interests for $\varphi$-XANES analysis (dashed boxes).

Summary of pooled spectra for young $(n=6)$, aged $(n=4)$ TJ1060 animals and aged animals with SIH $(n=4)$ or Lip-1 treatment $(n=5)$ (i.e. the mean for all pixels in all ROIs within each group) from scanning the iron K-edge, including features present in the pre-edge $(\sim 7.112 \mathrm{keV})$ and rising edge $(\sim 7.124 \mathrm{keV})$ are shown in Fig S3A, with their corresponding first-derivatives (Fig S3B). Similarly, pooled spectra and first derivatives from 4 day old adult wild types treated with $(n=4)$ and without $(n=4)$ DEM is shown in Fig S3C-D. 
A

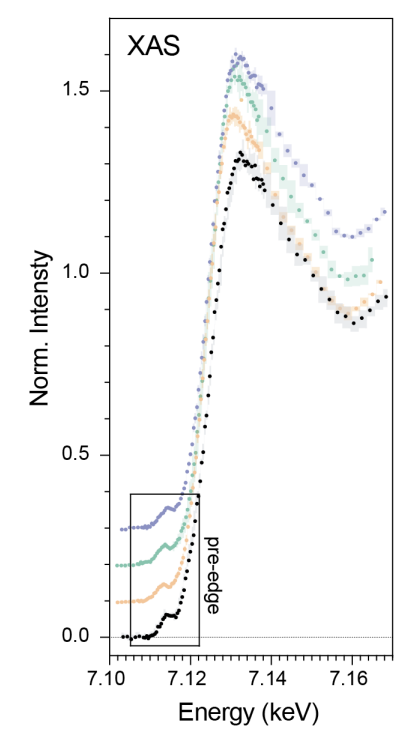

B

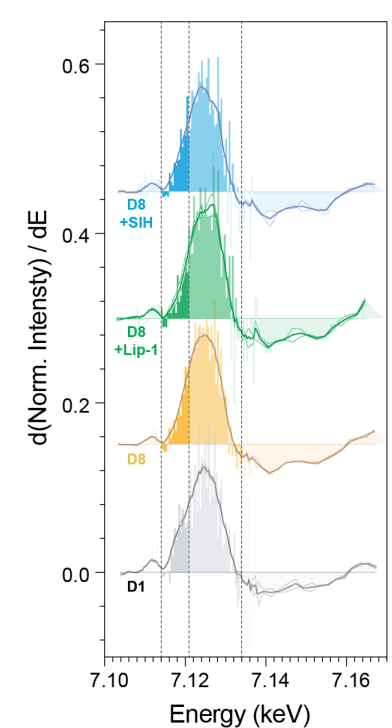

C

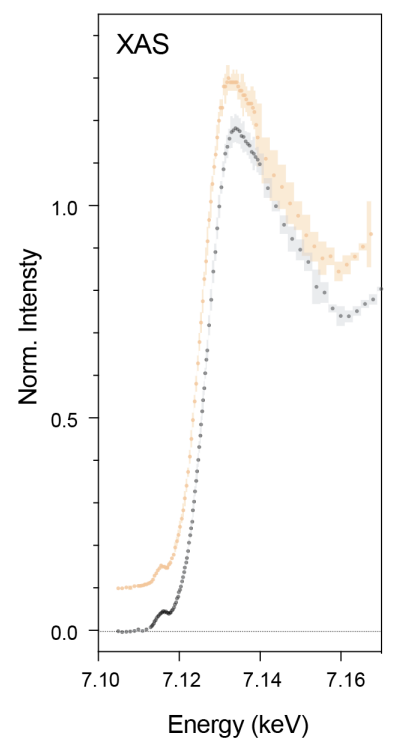

D

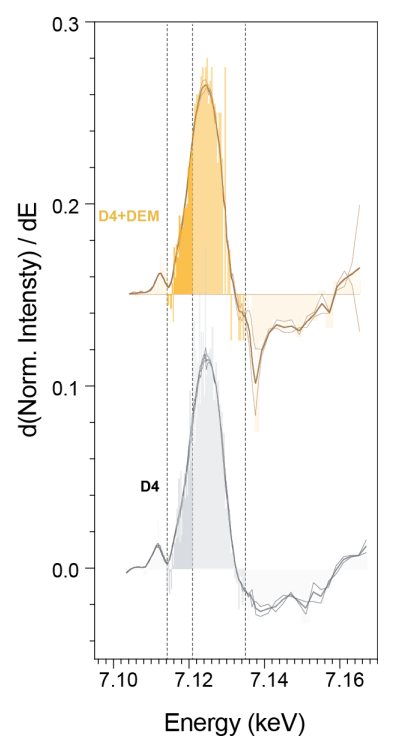

Figure S3: Changes in the first derivative of the Fe K-edge XANES reflect variation in the intensity of the $1 \mathrm{~s} \rightarrow 4 \mathrm{~s}$ and $1 \mathrm{~s} \rightarrow 4 \mathrm{p}$ transitions. A) Iron K-edge $\varphi$ XANES spectra (circles) determined by integrating 105 XFM micrographs acquired at energies spanning the iron absorption edge (7000 - 7455 eV). Shaded region represents $95 \%$ CI; spectra offset for clarity. Shown are spectra for Day 1 adults, Day 8 adults, Day $8+$ Lip and Day $8+$ SIH groups. B) The corresponding first derivative for spectra shown in $\mathbf{A}$; spectra offset in the vertical for clarity. Darker shading $(7.114-7.121 \mathrm{keV})$ marks the area under the curve attributed the $1 \mathrm{~s} \rightarrow 4 \mathrm{~s}$ transition. The lighter shading $(7.121-7.132 \mathrm{keV})$ marks the are under the curve attributed to the $1 \mathrm{~s} \rightarrow 4 \mathrm{p}$ transition. C) Pooled spectra for Day 4 adults treated with and without DEM, and $\mathbf{D}$ ) their matching first derivatives, as described above.

\section{Estimation of $\mathrm{Fe}^{2+}$}

There was a significant difference between the fractional $\mathrm{Fe}^{2+} / \mathrm{Fe}$ (total) estimates, determined by nonoverlapping 95\% CI, between aged TJ1060 animals (Table S7). Treatment with Lip-1 or SIH restored the $\mathrm{Fe}^{2+} / \mathrm{Fe}$ (total) estimate. Similarly, treatment of wild type (N2) animals with DEM markedly increased $\mathrm{Fe}^{2+} / \mathrm{Fe}($ total).

Table S7: Summary of the estimated $\mathrm{Fe}^{2+} / \mathrm{Fe}($ total) for each treatment group.

\begin{tabular}{|l|l|c|c|}
\hline & Group & $\mathrm{Mean} \mathrm{Fe}^{2+} / \mathrm{Fe}($ total $)$ & $95 \% \mathrm{CI}$ \\
\hline TJ1060 & Day 1 & 0.239 & $0.233-0.244$ \\
\hline & Day 8 Control & 0.300 & $0.295-0.305$ \\
& Day 8 Lip-1 & 0.231 & $0.198-0.262$ \\
\hline & Day 8 SIH & 0.236 & $0.232-0.240$ \\
\hline N2 & & & \\
\hline & Day 4 & 0.228 & $0.223-0.233$ \\
& Day 4 + DEM & 0.310 & $0.305-0.314$ \\
\hline
\end{tabular}




\section{Lifespan analysis}

Table S8: Summary of survival data from 8 independent replicate experiments. Median and maximum lifespan figures are days of adulthood at $25( \pm 1)^{\circ} \mathrm{C}$. Censored individuals are those that were lost, primarily due to crawling off the side of the plate. Median lifespan was initially compared using a Log-rank (Mantel-Cox) test. * $<<0.0001 ; \uparrow p=0.0013$

\begin{tabular}{|c|c|c|c|c|c|c|c|c|c|c|c|c|c|c|}
\hline \multirow[b]{2}{*}{ Replicate } & \multicolumn{4}{|c|}{ control } & \multicolumn{5}{|c|}{$250 \mu \mathrm{M}$ SIH } & \multicolumn{5}{|c|}{$200 \mu \mathrm{M}$ Lip-1 } \\
\hline & $\begin{array}{c}\text { death } \\
\text { events }\end{array}$ & censored & $\begin{array}{c}\text { med } \\
\text { ian }\end{array}$ & max. & $\begin{array}{c}\text { death } \\
\text { events }\end{array}$ & censored & median & $\max$. & $\begin{array}{c}\% \\
\text { median } \Uparrow\end{array}$ & $\begin{array}{c}\text { death } \\
\text { events }\end{array}$ & censored & median & $\max$. & $\begin{array}{c}\% \% \\
\text { median } \Uparrow\end{array}$ \\
\hline 1 & 88 & 3 & 7 & 17 & 71 & 4 & $14^{*}$ & 19 & 100 & 103 & 1 & $13^{*}$ & 25 & 86 \\
\hline 2 & 61 & 10 & 8 & 16 & 96 & 7 & $14^{*}$ & 19 & 75 & 88 & 6 & $11^{\dagger}$ & 20 & 38 \\
\hline 3 & 109 & 3 & 9 & 21 & 81 & 7 & $16^{*}$ & 23 & 78 & 108 & 2 & $14^{*}$ & 24 & 56 \\
\hline 4 & 99 & 1 & 7 & 19 & 111 & 10 & $16^{*}$ & 20 & 129 & 147 & 1 & $14^{*}$ & 24 & 100 \\
\hline 5 & 91 & 16 & 9 & 19 & 93 & 9 & $14^{*}$ & 18 & 56 & 86 & 7 & $14^{*}$ & 26 & 56 \\
\hline 6 & 97 & 11 & 8 & 16 & 105 & 10 & $14^{*}$ & 24 & 75 & 112 & 6 & $12^{*}$ & 25 & 50 \\
\hline 7 & 93 & 2 & 7 & 21 & 90 & 29 & $17^{*}$ & 29 & 143 & 80 & 6 & $15^{*}$ & 27 & 114 \\
\hline 8 & 72 & 5 & 8 & 22 & 73 & 13 & $20^{*}$ & 26 & 150 & 85 & 5 & $14^{*}$ & 26 & 75 \\
\hline Total & 709 & 51 & & & 720 & 89 & & & & 809 & 34 & & & \\
\hline Mean & & & 8 & 19 & & & 16 & 22 & $+101 \%$ & & & 13 & 25 & $+72 \%$ \\
\hline
\end{tabular}


A

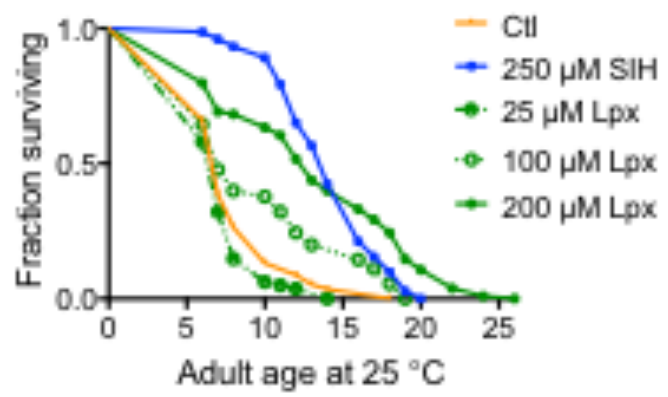

C

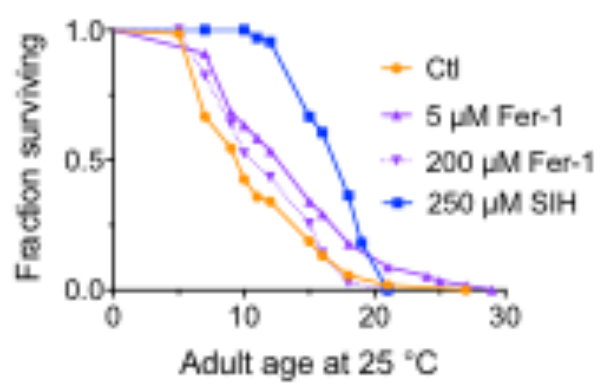

$\mathbf{E}$

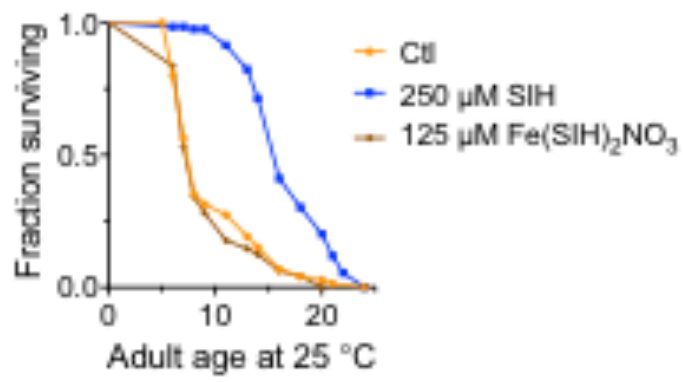

$\mathbf{B}$

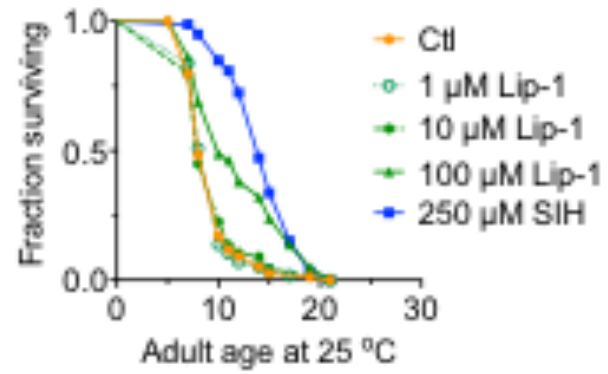

D

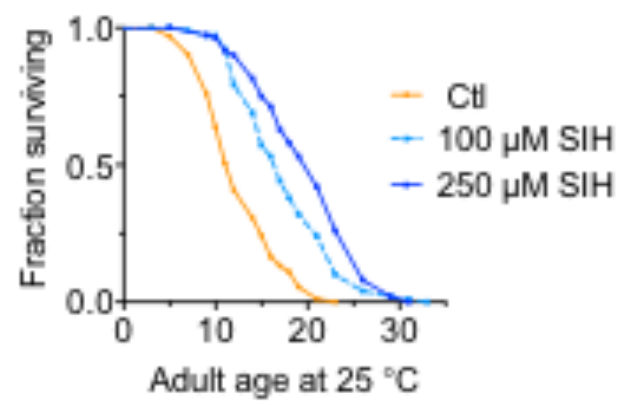

Figure S4: Dose response of SIH, Liproxstatin-1 and Ferrostatin-1

A: Median survival (Log-rank (Mantel-Cox) test: Control=6 days; $25 \mu \mathrm{M} \mathrm{Lip}-1=6$ days ( $p=0.07 \mathrm{~ns}$ ); $100 \mu \mathrm{M}$ Lip- $1=6$ days $(p=0.006) ; 200 \mu \mathrm{M}$ Lip- $1=12$ days $(p<0.0001) ; 250 \mu \mathrm{M} \mathrm{SIH}=13$ days $(p<0.0001)$

B: Median survival (Log-rank (Mantel-Cox) test: Control=7 days; $1 \mu \mathrm{M} \mathrm{Lip-1=9} \mathrm{days} \mathrm{(} p=0.90 \mathrm{~ns}) ; 10$ $\mu \mathrm{M}$ Lip-1=7 days $(p=0.78 \mathrm{~ns}) ; 100 \mu \mathrm{M}$ Lip-1=9 days $(p<0.0001) ; 250 \mu \mathrm{M}$ SIH=13 days $(p<0.0001)$

C: Median survival (Log-rank (Mantel-Cox) test: Control=10 days; $5 \mu \mathrm{M}$ Fer-1=15 days ( $p=0.0047)$; $200 \mu \mathrm{M}$ Fer- $1=12$ days $(p=0.35 \mathrm{~ns}) ; 250 \mu \mathrm{M} \mathrm{SIH}=18$ days $(p<0.0001)$

D: Median survival (Log-rank (Mantel-Cox) test: Control=12 days; $100 \mu \mathrm{M} \mathrm{SIH=17} \mathrm{days}(p<0.0001)$; $250 \mu \mathrm{M} \mathrm{SIH}=21$ days $(p<0.0001)$

E: Median survival (Log-rank (Mantel-Cox) test: Control=8 days; $125 \mu \mathrm{M} \mathrm{Fe}(\mathrm{SIH})_{2} \mathrm{NO}_{3}$, equimolar $\mathrm{SIH}=8$ days $(p=0.51 \mathrm{~ns}) ; 250 \mu \mathrm{M}$ SIH $=16$ days $(p<0.0001)$ 
bioRxiv preprint doi: https://doi.org/10.1101/594408; this version posted March 31, 2019. The copyright holder for this preprint (which was not certified by peer review) is the author/funder. All rights reserved. No reuse allowed without permission.

\section{Testing Departure from Temporal Rescaling}

Following the recently published results of Stroustrup et al. ${ }^{37}$ we determined whether the results observed with both the SIH and Liproxstatin interventions were due to temporal scaling of aging.

Using the modified Kolmogorov-Smirnov (K-S) test ${ }^{71}$ we examined whether the treatment effects can be reasonably modelled using the Accelerated Failure Time (AFT) model to determine whether we can reasonably assume that the treatment effect manifests in temporal rescaling. To control for interreplicate differences, the test is conducted on the residuals a replicate-specific AFT model with the Buckley-James method ${ }^{72}$ using the nonparametric baseline hazards form. The function $b j$ in $\mathrm{R}$ package $r m s$ was used to fit the models. The null hypothesis for the two-sample K-S test is that the simple temporal rescaling holds and the residuals for the two treatment groups under comparison come from the same distribution.

Using the modified Kolmogorov-Smirnov (K-S) test, we examined whether the treatment effects can be reasonably modelled using the AFT model to determine whether we can reasonably assume that the treatment effect manifests in temporal rescaling. To control for inter-replicate differences, the test is conducted on the residuals from replicate-specific AFT model with the Buckley-James method that uses nonparametric baseline hazards form. The function $b j$ in $\mathrm{R}$ package $r m s$ was used to fit the models. The null hypothesis for the two-sample K-S test is that the simple temporal rescaling holds and the residuals for the two treatment groups under comparison come from the same distribution.

Since the $\mathrm{R}$ function can only take right-censored data and our lifespan data are interval-censored, we use the mid-point of the interval to assign the time of event. Treating interval-censored data as rightcensored is expected to underestimate the variability in the statistical estimates ${ }^{73}$ which in turn will produce an optimistic (smaller than it should be) $p$-value. To reduce the likelihood of false rejection of the null hypothesis merely because of the optimistic $p$-value, we chose a more stringent Type I error (0.01) than the usual 0.05 when conducting the K-S test.

Table S9: $p$-values of KS test on Residuals of nonparametric AFT models

\begin{tabular}{|r|r|r|r|}
\hline Replicate & Control vs Lip-1 & Control vs SIH & Lip-1 vs SIH \\
\hline 1 & $9 \times 10^{-5}$ & $2 \times 10^{-3}$ & $1 \times 10^{-15}$ \\
\hline 2 & $2 \times 10^{-2}$ & $4 \times 10^{-3}$ & $8 \times 10^{-5}$ \\
\hline 3 & $7 \times 10^{-3}$ & $9 \times 10^{-4}$ & $4 \times 10^{-11}$ \\
\hline 4 & $4 \times 10^{-13}$ & $3 \times 10^{-15}$ & $2 \times 10^{-31}$ \\
\hline 5 & $2 \times 10^{-4}$ & $1 \times 10^{-4}$ & $7 \times 10^{-28}$ \\
\hline 6 & $1 \times 10^{-9}$ & $3 \times 10^{-5}$ & $2 \times 10^{-5}$ \\
\hline 7 & $3 \times 10^{-6}$ & $4 \times 10^{-14}$ & $1 \times 10^{-7}$ \\
\hline 8 & $1 \times 10^{-6}$ & $4 \times 10^{-6}$ & $1 \times 10^{-8}$ \\
\hline
\end{tabular}

As can be seen from Table S9, the effect of Lip-1 and SIH treatment relative to control always deviates away from simple temporal rescaling (all $p$-values $<10^{-2}$ ) with the exception of Lip-1 in replicate 2. Figure S5 shows graphically why the AFT assumption is not reasonable since the survival curves of residuals from the AFT models show 'crossing' behavior. If the simple temporal rescaling assumption is reasonable, we would expect the survival curves for the different treatments to be very similar to each other. The observed crossing of the curves is primarily caused by the de-acceleration in the survival function for control worms.

When all the replicates are combined, and the modified KS test were performed on the residuals of the AFT models with the best parametric form, we found that the $p$-value for comparing Control vs Lip-1, Control vs SIH and Lip-1 vs SIH are $2 \times 10^{-24}$, $1 \times 10^{-24}$ and $3 \times 10^{-37}$ respectively. These results indicate that failure to control for inter-replicate differences would lead to even stronger evidence of departure from simple temporal rescaling. 

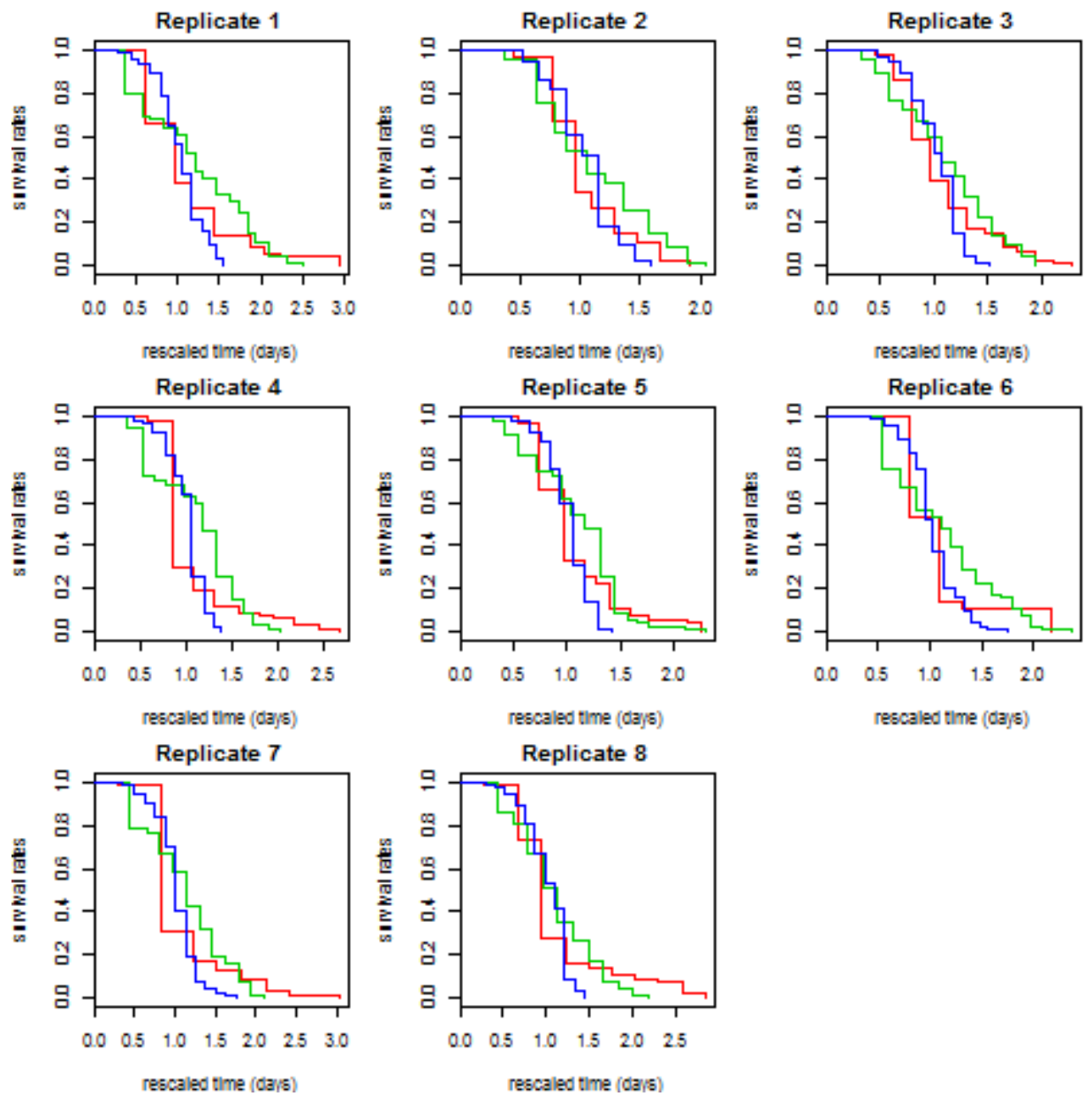

Figure S5: The residuals of AFT model with nonparametric hazards form for different replicates (red $=$ control, green $=$ Lip-1, blue $=\mathrm{SIH})$. The $\mathrm{x}$-axis has been rescaled to remove the temporal rescaling effect. Under the null hypothesis, we expect the residuals for the different groups to have the same survival curves.

\section{Determining AFT models with the best baseline hazard form}

In order to investigate the possible reasons for departure from simple temporal rescaling, we used parametric survival models which require specification of a parametric baseline hazard form. To minimize the risk of model misspecification, we identified the most appropriate baseline hazard form for each replicate using the Bayesian Information Criterion (BIC), with the best parametric form chosen as the model that minimizes the BIC.

The following parametric baseline hazards were fitted:

Gompertz, Gompertz with Frailty, Weibull, Weibull with Frailty, Log-normal and Log-logistic. The mathematical formulae for each parametric form are detailed below:

Gompertz: $\mathrm{h}(\mathrm{t} \mid \mathrm{a}, \mathrm{b})=(\mathrm{a} / \mathrm{b}) \exp (\mathrm{t} / \mathrm{b})$

Gompertz with frailty: $h(t \mid a, b, \sigma)=(a / b) \exp (t / b) /\left[1+\sigma^{2} a \exp ((t / b)-1)\right]$

Weibull: $\mathrm{h}(\mathrm{t} \mid \alpha, \beta)=(\alpha / \beta)(\mathrm{t} / \beta)^{\alpha-1}$

Weibull with frailty: $\mathrm{h}(\mathrm{t} \mid \alpha, \beta, \sigma)=(\alpha /)(\mathrm{t} / \beta)^{\alpha-1} /\left[1+\sigma^{2}(\mathrm{t} / \beta)^{\alpha}\right]$

Log-normal: $\mathrm{h}(\mathrm{t} \mid \mu, \sigma)=\phi((\log \mathrm{t}-\mu) / \sigma) / \sigma \mathrm{t}[1-\Phi((\log \mathrm{t}-\mu) / \sigma)]$

Log-logistic: $h(t \mid \alpha, \beta)=(\alpha / \beta)(t / \beta)^{\alpha-1}\left[1+(t / \beta)^{\alpha}\right]$ 
Here $\phi$ and $\Phi$ denote the probability density function (PDF) and cumulative distribution function (CDF), respectively, of the standard normal distribution; $\mu$ and $\sigma$ denote the mean and standard deviation (in the case of the log-normal, the mean and standard deviation of the logarithm of $x$ ); $\lambda$, $\alpha$, and $a$ are shape parameters; $\beta$ and $b$ are scale parameters. In the case of frailty, individual hazards $h_{i}(t)$ are related to a baseline hazard by a random factor $Z$ that follows a Gamma distribution with mean 1 and variance $\sigma^{2}$.

Bayesian Information Criterion (BIC) is used to determine the best parametric form of the hazards; with better fit indicated by lower BIC value. All computations are done using flexsurv R package, taking into account that events are interval censored to account for the fact that we do not observe the exact event time and only know that events occurred within an interval $(a, b)$.

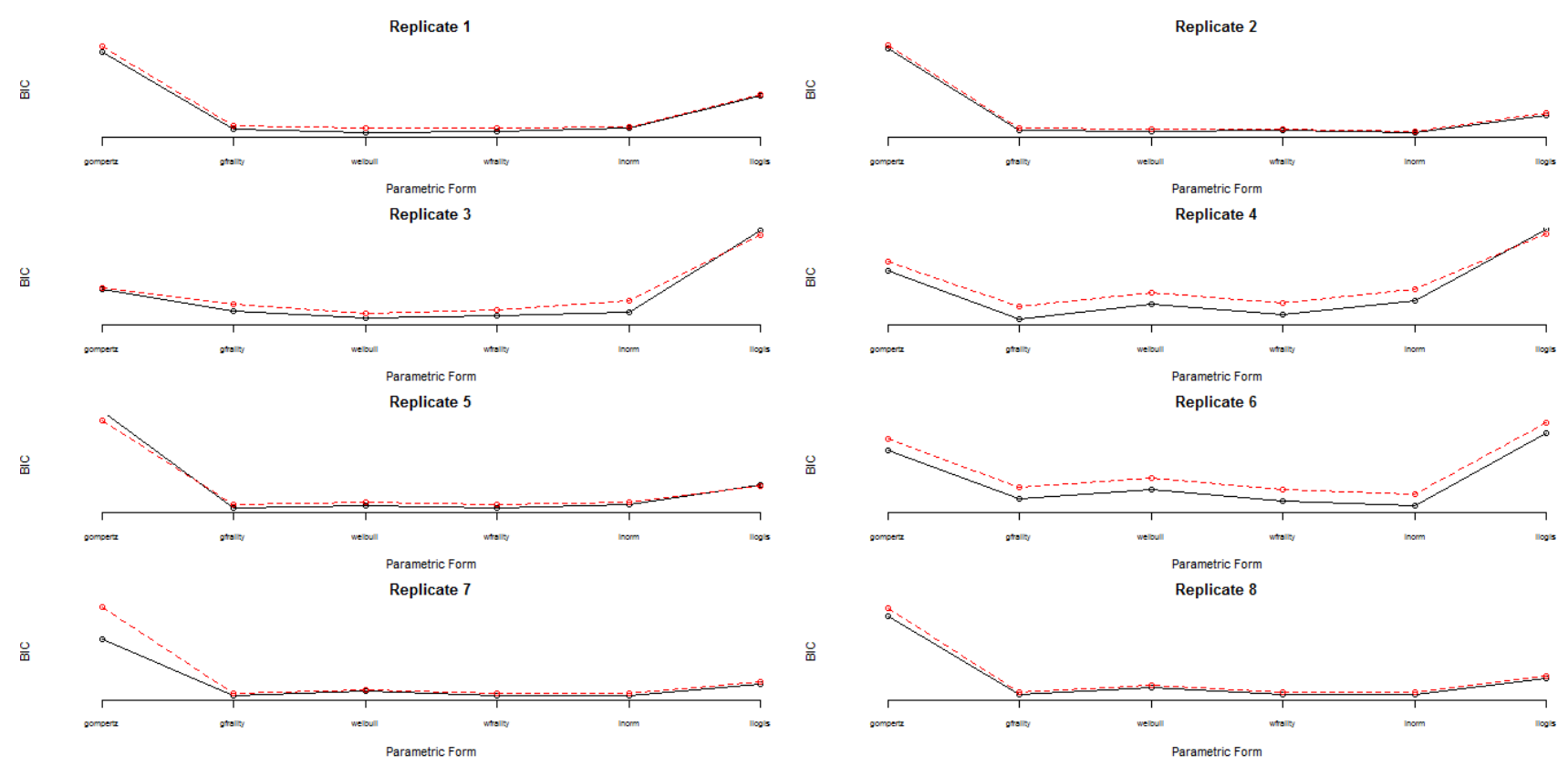

Figure S6: Bayesian Information Criterion (BIC) for AFT models with different parametric baseline hazard forms

From Figure S6, we can see that Gompertz baseline hazard form does not fit the data well, except when frailty is used. Weibull baseline hazard fits some replicates quite well and the fit is further improved when frailty is assumed. In fact, Weibull with frailty provides the best parametric baseline hazards form for nearly all the replicates, followed closely by the log-normal models.

\section{Possible Causes of Departure from Temporal Rescaling}

Stroustrup et al. ${ }^{37}$ show that unobserved heterogeneity (e.g. due to heterogeneity in the temperature the worms were exposed to) could cause de-acceleration and further, when the degree of heterogeneity is different between treatments, this could give rise to apparent departure from temporal rescaling. We investigated whether there is significant difference in the degree of heterogeneity by comparing two models for each replicate: (M1) model with Weibull frailty (Weibull hazard, Gamma frailty) where the degree of heterogeneity (represented by parameter $\sigma^{2}$ and $a$ ) is assumed to be the same for all three treatments, (M2) where the parameter $\sigma^{2}$ is allowed to be different but parameter $a$ fixed across treatments and (M3) where the parameter $\sigma^{2}$ and a are allowed to be different across treatments. We compared the three models based on their BIC values and also performed likelihood ratio tests, comparing M1 vs M2 and M1 vs M3.

Note that only M1 can be classified as an AFT model while M2 and M3 are not AFT models, as the treatment effects also manifest in the other parameters apart from the location (shift) parameter. 
Table S10 shows that both M3 and M2 provide better fit than M1 for all replicates as indicated by small likelihood ratio test (LRT) p-values, with M3 providing more convincing p-values.

Table S10 BIC values for AFT model with Weibull frailty baseline hazards (M1), non-AFT model with Weibull frailty baseline hazards and treatment-dependent heterogeneity levels $\sigma^{2}(\mathrm{M} 2)$ and nonAFT model with Weibull frailty baseline hazards and treatment-dependent shape parameter (a) and heterogeneity levels $\sigma^{2}(\mathrm{M} 3)$

\begin{tabular}{|r|c|c|c|r|r|}
\hline Replicate & M1 & M2 & M3 & $\begin{array}{r}\text { LRT P-value } \\
(\mathrm{M} 2 \text { vs M1) }\end{array}$ & $\begin{array}{r}\text { LRT P-value } \\
(\mathrm{M} 3 \text { vs M1) }\end{array}$ \\
\hline 1 & 1325.3 & 1321.0 & 1289.4 & $2 \times 10^{-4}$ & $2 \times 10^{-12}$ \\
\hline 2 & 1090.3 & 1080.7 & 1081.8 & $1 \times 10^{-5}$ & $1 \times 10^{-6}$ \\
\hline 3 & 1503.6 & 1495.5 & 1476.0 & $3 \times 10^{-5}$ & $8 \times 10^{-11}$ \\
\hline 4 & 1613.3 & 1590.7 & 1500.0 & $1 \times 10^{-8}$ & 0 \\
\hline 5 & 1263.5 & 1246.4 & 1224.0 & $3 \times 10^{-7}$ & $2 \times 10^{-13}$ \\
\hline 6 & 1464.3 & 1431.8 & 1408.2 & $8 \times 10^{-11}$ & 0 \\
\hline 7 & 1079.0 & 1066.4 & 1034.1 & $3 \times 10^{-6}$ & $1 \times 10^{-14}$ \\
\hline 8 & 1033.3 & 1016.0 & 1004.0 & $3 \times 10^{-7}$ & $5 \times 10^{-11}$ \\
\hline
\end{tabular}

The fitted survival curves for M3 model in each replicate are compared to the observed curves in Figure S7. To investigate whether M3 provides an adequate fit to the data, for each replicate, we performed a chi-square goodness of fit test, comparing the observed survival curve to the fitted curve for each treatment group. The results are presented in Table S11. While the controls and SIH are always well-fitted by the Weibull frailty models (all $p$-values $>0.01$ ), the Lip-1 data from replicates 1 , 4, 5 and 7 are not adequately fitted by the Weibull frailty model. The lack of fit for replicate 4 in particular is mainly caused by the estimated survival underestimating the observed counterparts in the middle-section between 7 and 15 days and overestimation on the tails.

Table S11 Chi-square Goodness of Fit $p$-value for M3 (non-AFT model with treatment-dependent shape and heterogeneity parameters)

\begin{tabular}{|r|c|c|c|}
\hline Replicate & Control & Lip-1 & SIH \\
\hline 1 & 0.4 & $4 \times 10^{-4}$ & 0.8 \\
\hline 2 & 0.08 & 0.07 & 0.1 \\
\hline 3 & 0.05 & 0.08 & 0.1 \\
\hline 4 & 0.05 & $1 \times 10^{-12}$ & 0.5 \\
\hline 5 & 0.4 & $2 \times 10^{-5}$ & 0.8 \\
\hline 6 & 0.001 & 0.01 & 0.2 \\
\hline 7 & 0.2 & 0.001 & 0.4 \\
\hline 8 & 0.1 & 0.07 & 0.07 \\
\hline
\end{tabular}



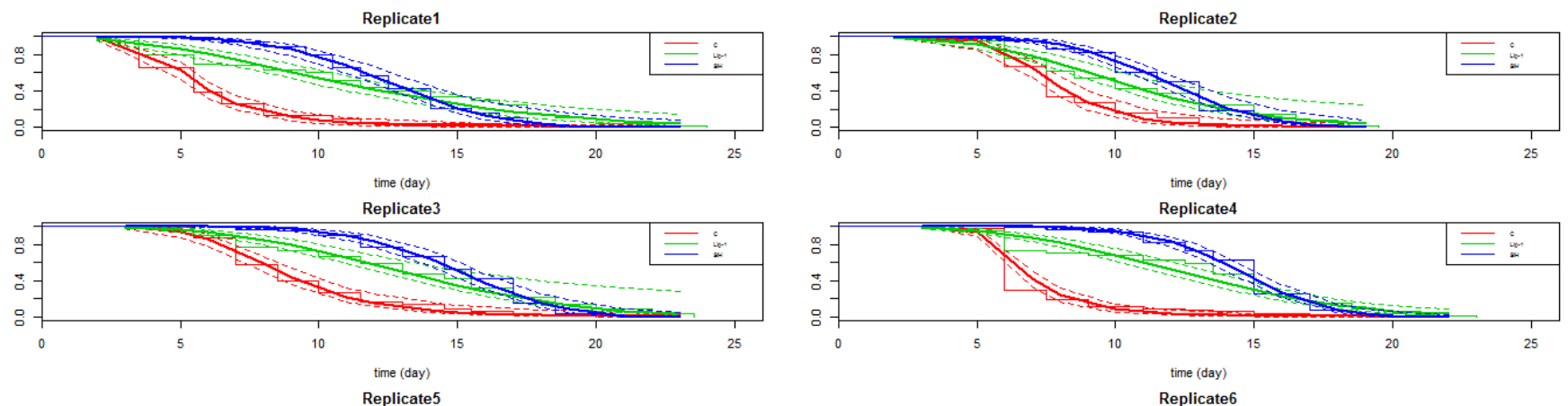

Replicate5
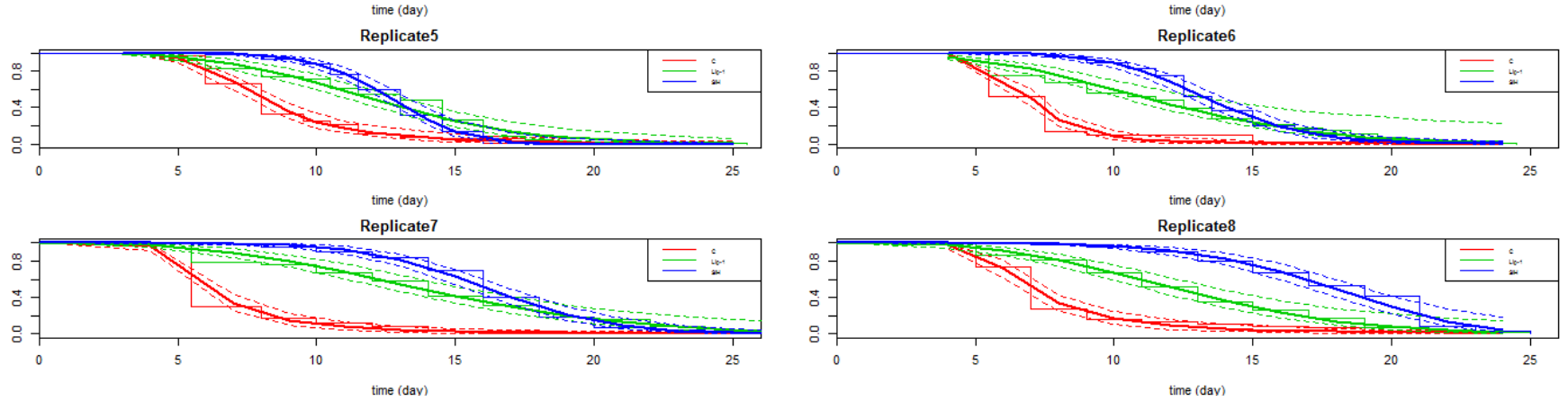

Figure S7: The observed and fitted survival curves based on the best non-AFT model for each replicate

\section{Combining Replicates}

\section{Models for Combining Similar Replicates}

We tried to identify the most parsimonious model that can best fit the combined data from all replicates. If some of the parameters are quite similar across replicates, we can fit a simpler model than the saturated model where all parameters are allowed to be different across replicates. A range of models are fitted and the best simple model for the combined data is Model 6 (M6) with the same treatment-dependent shapes and heterogeneity parameters across replicates but replicate-specific parameters for scale parameter of the control worms and temporal rescaling parameters. The BIC value for this model is smaller than that for the saturated model (15079 vs 15347) and the LR test statistic is $79.2(\mathrm{df}=90)$ with $p$-value $=0.78$, indicating that based on LR test the combined model (M6) does not provide worse fit to the data. The need for replicate-specific scale parameters and temporal rescaling parameters corroborates the evidence in Figure S7 which showed these parameters as having considerable variations across replicates.

Goodness-of-fit (GOF) test at replicate-level based on model M6 (Table S12) shows that this model provides more or less the same level of fit to the replicate-specific model (Table S11), with replicates showing good fit before still showing good fit now. The fitted survival curves (based on M6) are given in Figure S8.

Table S12 Chi-square Goodness of Fit Statistics ( $p$-values) for M6 (the best parsimonious model according to BIC)

\begin{tabular}{|r|r|r|r|}
\hline Replicate & Control & Lip-1 & SIH \\
\hline 1 & $6 \times 10^{-1}$ & $10^{-6}$ & $5 \times 10^{-1}$ \\
\hline 2 & $2 \times 10^{-1}$ & $5 \times 10^{-2}$ & $4 \times 10^{-2}$ \\
\hline 3 & $9 \times 10^{-3}$ & $10^{-1}$ & $2 \times 10^{-1}$ \\
\hline 4 & $10^{-2}$ & $4 \times 10^{-11}$ & $2 \times 10^{-1}$ \\
\hline 5 & $5 \times 10^{-1}$ & $4 \times 10^{-5}$ & $4 \times 10^{-1}$ \\
\hline 6 & $3 \times 10^{-7}$ & $10^{-2}$ & $4 \times 10^{-2}$ \\
\hline 7 & $3 \times 10^{-1}$ & $3 \times 10^{-3}$ & $5 \times 10^{-1}$ \\
\hline 8 & $2 \times 10^{-1}$ & $2 \times 10^{-1}$ & $7 \times 10^{-2}$ \\
\hline
\end{tabular}



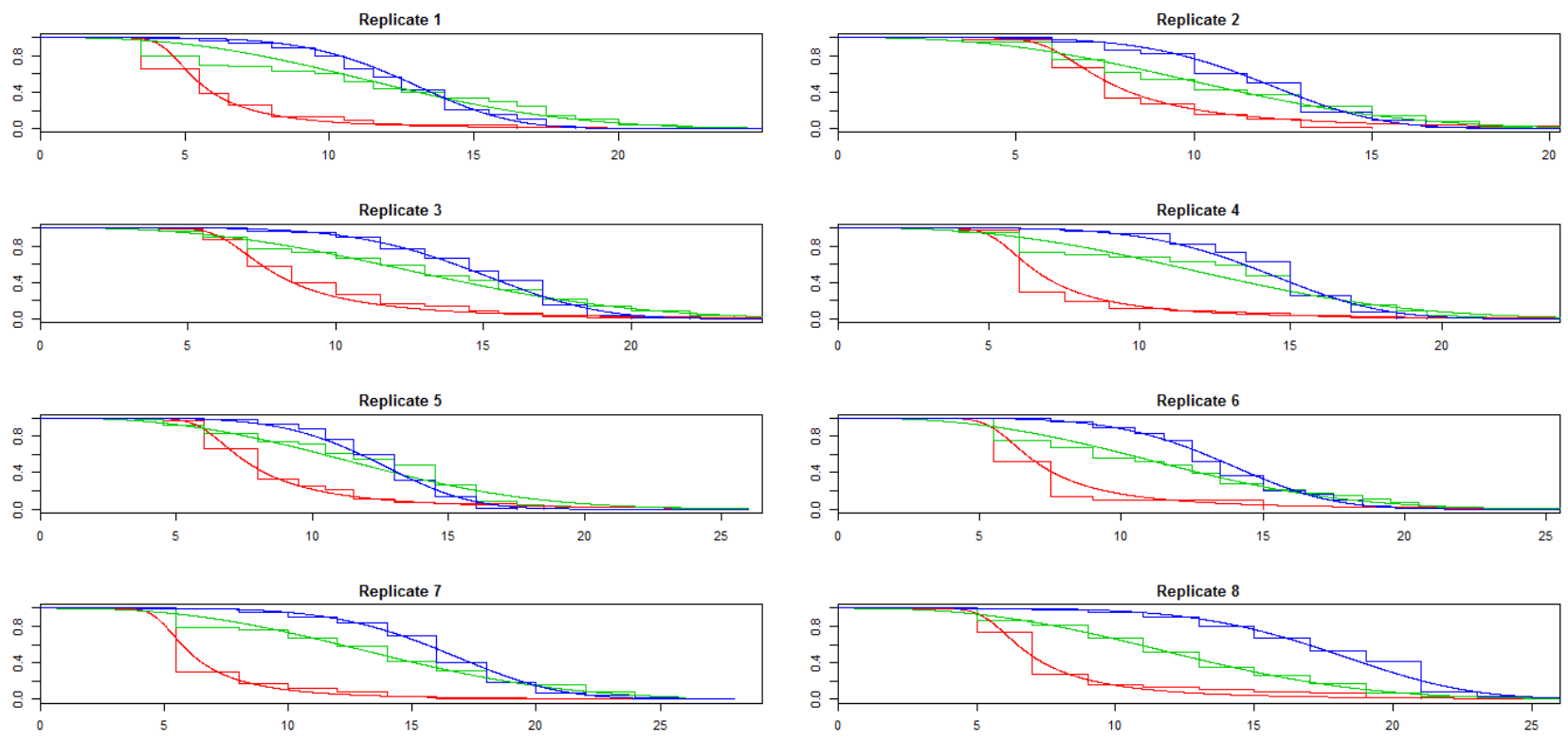

Figure S8: The observed and fitted survival curves based on model the most parsimonious model M6

\section{Meta-Analysis}

In Table S13, we present the parameter estimates from the best non-AFT model with Weibull frailty hazards for each replicate and also fixed-effect and random-effect meta-analysis estimates for each parameter of the model. Briefly, the fixed-effect meta-analysis estimates were derived using Inverse Variance Weighting (IVW) in which the estimates from each replicate were weighted by the inverse of the variance estimates. The meta-analysis estimates were then calculated simply as the weighted average of estimates from all replicates. The fixed-effect meta-analysis assumes there is insignificant variation in the estimates of the same parameter across different replicates. The random-effect meta-analysis derived the estimates by also assigning weights to estimates from each replicate, but the weights take into account the variation of estimates across replicates.
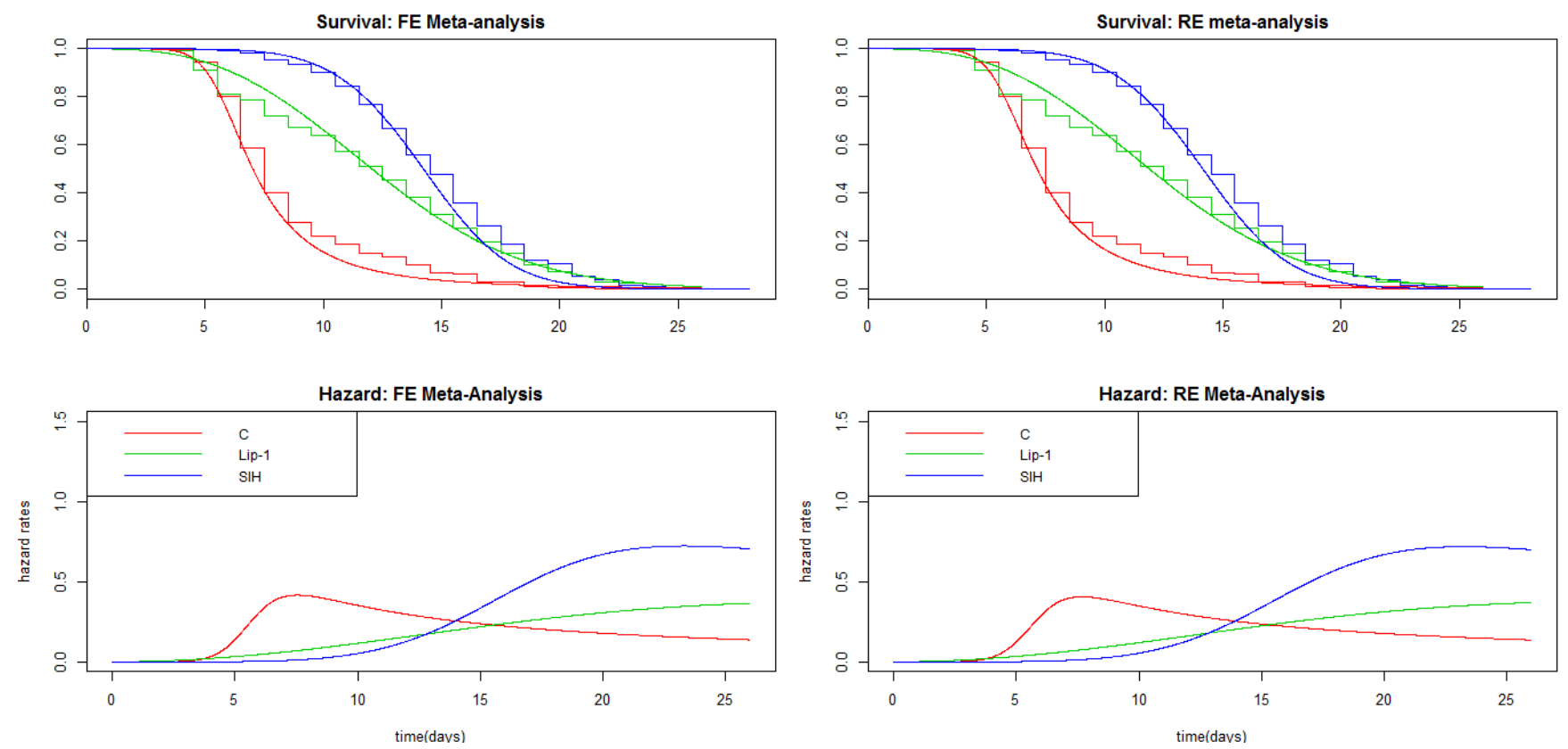

Figure S9: Meta-analysis Estimates of Survival and Hazard Functions. FE=fixed error, RE=random error. 
Table S13: Parameter Estimates and their 95\% confidence intervals from non-AFT Weibull-Frailty Models with treatment-dependent shape and heterogeneity parameters

\begin{tabular}{|c|c|c|c|c|c|c|c|c|c|}
\hline Replicate & $\log \left(a_{C}\right)$ & $\log \left(b_{C}\right)$ & $\log \left(\sigma^{2} c\right)$ & $\theta_{\text {Lip-1 }}^{1}$ & $\theta^{1}$ SIH & $\Delta \log \left(\mathrm{a}_{\text {Lip-1 }}\right)$ & $\Delta \log \left(\mathrm{a}_{\mathrm{SIH}}\right)$ & $\Delta \log \left(\sigma_{\text {Lip- } 1}^{2}\right)$ & $\Delta \log \left(\sigma^{2} \mathrm{SIH}\right)$ \\
\hline 1 & $1.73(1.1 ; 2.36)$ & $1.67(1.55 ; 1.8)$ & $0.24(-0.24 ; 0.71)$ & $2.37(2.01 ; 2.8)$ & $2.5(2.16 ; 2.89)$ & $-1.02(-1.67 ;-0.36)$ & $-0.17(-0.85 ; 0.51)$ & $-1.67(-2.91 ;-0.44)$ & $-1.15(-2.35 ; 0.05)$ \\
\hline 2 & $1.9(1.25 ; 2.55)$ & $2.04(1.91 ; 2.17)$ & $0.04(-0.61 ; 0.7)$ & $1.41(1.13 ; 1.76)$ & $1.62(1.4 ; 1.88)$ & $-0.74(-1.48 ;-0.01)$ & $-0.23(-0.92 ; 0.47)$ & $-0.57(-1.84 ; 0.69)$ & $-0.79(-1.91 ; 0.32)$ \\
\hline 3 & $1.65(1.13 ; 2.17)$ & $2.14(2 ; 2.29)$ & $0.03(-0.55 ; 0.61)$ & $1.7(1.42 ; 2.04)$ & $1.86(1.6 ; 2.17)$ & $-0.54(-1.09 ; 0.02)$ & $0.16(-0.39 ; 0.72)$ & $-1.12(-2.75 ; 0.51)$ & $-0.97(-2.01 ; 0.07)$ \\
\hline 4 & $2.47(2.04 ; 2.9)$ & $1.84(1.77 ; 1.9)$ & $0.5(0.22 ; 0.78)$ & $2.19(1.99 ; 2.4)$ & $2.4(2.23 ; 2.59)$ & $-1.39(-1.84 ;-0.93)$ & $-0.5(-0.97 ;-0.03)$ & $-1.66(-2.48 ;-0.83)$ & $-1.24(-1.94 ;-0.53)$ \\
\hline 5 & $1.91(1.42 ; 2.39)$ & $2.04(1.92 ; 2.16)$ & $0.27(-0.17 ; 0.71)$ & $1.71(1.48 ; 1.98)$ & $1.72(1.52 ; 1.96)$ & $-0.72(-1.25 ;-0.2)$ & $0.08(-0.45 ; 0.62)$ & $-1.18(-2.07 ;-0.29)$ & $-1.05(-2.06 ;-0.04)$ \\
\hline 7 & $2.26(1.76 ; 2.76)$ & $1.74(1.62 ; 1.85)$ & $0.53(0.18 ; 0.88)$ & $2.69(2.3 ; 3.15)$ & $2.94(2.58 ; 3.34)$ & $-1.23(-1.77 ;-0.69)$ & $-0.42(-0.98 ; 0.14)$ & $-1.44(-2.34 ;-0.53)$ & $-0.93(-1.51 ;-0.34)$ \\
\hline 8 & $2.19(1.7 ; 2.69)$ & $1.86(1.77 ; 1.96)$ & $0.51(0.17 ; 0.85)$ & $2.08(1.78 ; 2.42)$ & $2.96(2.66 ; 3.3)$ & $-1.08(-1.64 ;-0.53)$ & $-0.52(-1.05 ; 0.02)$ & $-1.31(-2.35 ;-0.27)$ & $-1.83(-3.03 ;-0.63)$ \\
\hline $\begin{array}{l}\text { Meta-analysis } \\
\text { (Fixed Effects) } \\
\text { ALL }\end{array}$ & $2.12(1.93 ; 2.30)$ & $1.88(1.85 ; 1.91)$ & $0.42(0.28 ; 0.56)$ & $2.04(1.94 ; 2.15)$ & $2.26(2.17 ; 2.35)$ & $-1.05(-1.26 ;-0.85)$ & $-0.29(-0.49 ;-0.08)$ & $-1.35(-1.72 ;-0.97)$ & $-1.01(-1.3 ;-0.72)$ \\
\hline $\begin{array}{l}\text { Meta-analysis } \\
\text { (Random } \\
\text { Effects) } \\
\text { ALL } \\
\end{array}$ & $2.11(1.86 ; 2.36)$ & $1.90(1.79 ; 2.00)$ & $0.42(0.28 ; 0.56)$ & $1.97(1.73 ; 2.27)$ & $2.20(1.88 ; 2.59)$ & $-1.05(-1.31 ;-0.79)$ & $-0.29(-0.51 ;-0.06)$ & $-1.35(-1.72 ;-0.97)$ & $-1.01(-1.3 ;-0.72)$ \\
\hline
\end{tabular}

${ }^{1} \theta_{\text {Lip- } 1}=b_{\text {Lip }-1} / b_{C} ; \theta_{\text {SIH }}=b_{\text {SIH }} / b_{\mathrm{C}}$

Conclusions from Table S13:

- There are between-replicate variations in the scaling factor estimates. But after combining across different replicates, $\boldsymbol{\theta}_{\text {sIH }}$ estimate is $2.20(95 \%$ CI: $1.88 ; 2.59)$ and $\theta_{\text {Lip-1 }}$ estimate is $1.97(95 \%$ CI: $1.73 ; 2.27)$, which means that life has significantly de-accelerated under Lip-1 and SIH relative to under controls, by about half.

- Meta-analysis estimates also shows that the treatment effect manifests in not only simple scaling of the Weibull scale parameter, but it also affects the shape parameters with the shape parameters for Lip-1 and SIH being significantly smaller (the 95\% CI for differences in log shape parameter between Lip-1 and SIH versus controls do not include zero).

- Meta-analysis estimates also shows there is significant heterogeneity due to unobserved factors among control worms, $95 \% \mathrm{CI}$ for log $\sigma^{2}{ }_{\mathrm{c}}=$ $0.42(95 \%$ CI: $0.28 ; 0.56)$ while the heterogeneity due to unobserved factors are significantly less in Lip-1 and SIH worms. 
Table S14: Comparison of Models for Combined Data

\begin{tabular}{|c|c|c|c|c|c|c|c|c|c|c|}
\hline Model & $\log (\mathbf{a})$ & $\log (b)$ & $\log \left(\sigma_{C}^{2}\right)$ & $\theta_{\text {Lip-1 }}$ & $\theta_{\mathrm{SIH}}$ & $\Delta \log \left(\mathbf{a}_{\text {Lip-1 }}\right)$ & $\Delta \log \left(\mathbf{a}_{\mathrm{SIH}}\right)$ & $\Delta \log \left(\sigma_{\text {Lip-1 }}^{2}\right)$ & $\Delta \log \left(\sigma_{\text {SIH }}^{2}\right)$ & BIC value \\
\hline M1 & same & different & same & same & same & 0 & 0 & 0 & 0 & 15835.49 \\
\hline $\mathrm{M} 2$ & same & different & same & different & different & 0 & 0 & 0 & 0 & 15887.84 \\
\hline M3 & same & different & same & same & same & 0 & 0 & same & same & 15490.08 \\
\hline M4 & same & different & same & different & different & 0 & 0 & same & same & 15511.38 \\
\hline M5 & same & different & same & same & same & same & same & same & same & 15109.10 \\
\hline M6 & same & different & same & different & different & same & same & same & same & 15079.13 \\
\hline M7 & different & different & different & different & different & same & same & same & same & 15250.78 \\
\hline Saturated & different & different & different & different & different & different & different & different & different & 15346.79 \\
\hline
\end{tabular}

same $=$ assumed the same across replicates

different $=$ allowed to be different across replicates

$0=$ not estimated, set to zero 


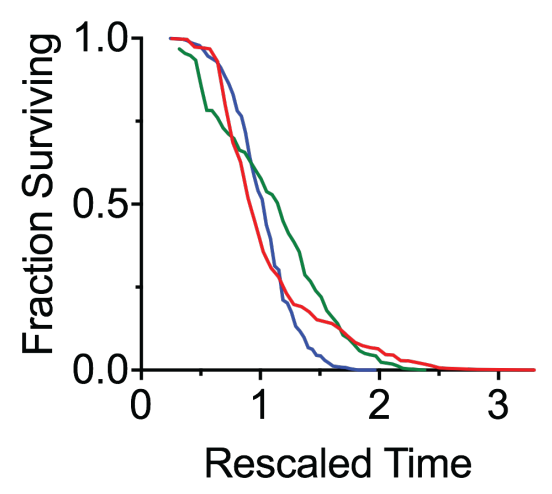

Figure S10: The best-fit AFT residuals from survival data meta-analysis shown in Figure S9, based the M6 model with fixed errors. Note that the survival curves cross rather than overlap, indicating temporal scaling is insufficient to explain the differences between treatments

\section{Investigating Temporal Rescaling by Temperature}

To investigate if changing the temperature showed evidence of temporal scaling we compared worms aged at $20^{\circ} \mathrm{C}$ and $25^{\circ} \mathrm{C}$ during the same time frame. The effect of temperature is evaluated separately for control and SIH treated worms, population sizes for each replicate are shown in Table S15.

Table S15: Population sizes for replicates used to assess temporal rescaling by temperature

\begin{tabular}{|c|c|c|c|}
\hline \multirow{2}{*}{ Replicate } & Temperature & $\begin{array}{c}\text { control } \\
\text { death } \\
\text { events }\end{array}$ & $\begin{array}{c}\text { SIH } \\
\text { death } \\
\text { events }\end{array}$ \\
\hline 1 & $20^{\circ} \mathrm{C}$ & 99 & 100 \\
\hline 1 & $25^{\circ} \mathrm{C}$ & 76 & 90 \\
\hline 2 & $20^{\circ} \mathrm{C}$ & 84 & 80 \\
\hline 2 & $25^{\circ} \mathrm{C}$ & 81 & 67 \\
\hline
\end{tabular}

\section{Checking AFT Assumption}

For each of two replicates and treatment factors, the AFT model with Weibull baseline hazard was fitted with temperature as the covariate. The residuals from this model were then subjected to the K-S test. The $p$-values from the K-S test are given in Table S16. As can be seen, the $p$-values are generally not very small (only one $p$-value $<0.01$ ), indicating that the evidence of departure from simple temporal rescaling is not strong. Figure $\mathbf{S 1 1}$ shows the survival curves of the residuals and despite the discreteness, the two distributions (black for $20^{\circ} \mathrm{C}$ and red for $25^{\circ} \mathrm{C}$ ) seem to be similar.

Table S16: $p$-values of KS test on Residuals of nonparametric AFT models

\begin{tabular}{|l|l|c|}
\hline Replicate & Treatment & $\boldsymbol{p}$-value \\
\hline 1 & Control & $2.0 \times 10^{-2}$ \\
\hline 1 & SIH & $3.5 \times 10^{-2}$ \\
\hline 2 & Control & $2.7 \times 10^{-3}$ \\
\hline 2 & SIH & $1.3 \times 10^{-1}$ \\
\hline
\end{tabular}


Control - replicate 1

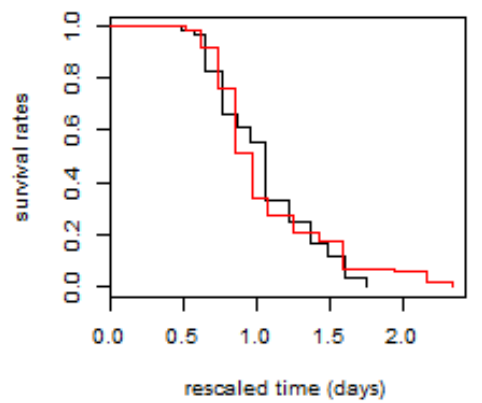

Control - replicate 2

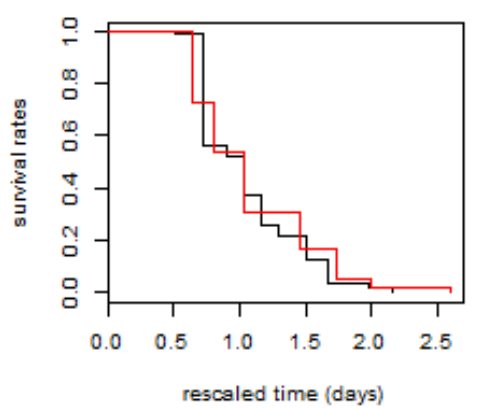

SIH - replicate 1

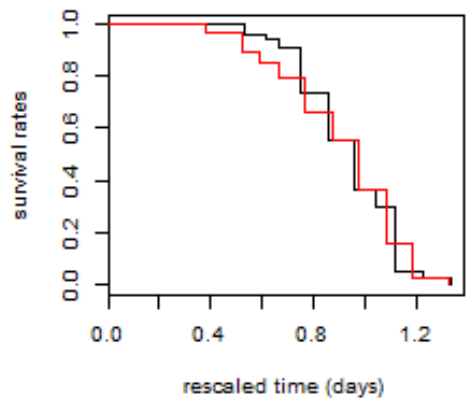

$\mathrm{SIH}$ - replicate 2

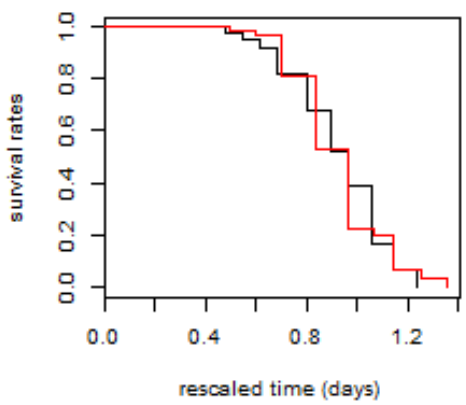

Figure S11: The best-fit AFT residuals from the model with Weibull baseline hazard for survival of control and SIH treated worms at $20{ }^{\circ} \mathrm{C}$ (black) and $25^{\circ} \mathrm{C}$ (red)

\section{Meta-Analysis}

We also performed meta-analysis for each worm population and the results are given below. The $\log \sigma^{2}$ provides an indication of the level of heterogeneity, and it is interesting to note that the control worms exhibit greater heterogeneity than the SIH worms, consistent with that observed at $25^{\circ} \mathrm{C}$.

For both populations, being exposed to the higher temperature of $25{ }^{\circ} \mathrm{C}$ accelerates life as expected, by approximately $30 \%$ for control worms $\left(\theta_{\text {temp }}=\mathbf{0 . 7 2}(\mathbf{9 5 \%}\right.$ CI: $\mathbf{0 . 6 8 ; 0 . 7 7 )}$ and $20 \%$ for SIH worms $\left(\theta_{\text {temp }}=\mathbf{0 . 8 1}\right.$ (95\% CI: 0.78;0.84).

Table S17: Meta-analysis results for control and SIH treated worms

\begin{tabular}{|c|c|c|c|c|}
\hline \multicolumn{5}{|l|}{ Control worms } \\
\hline Replicate & Log Shape & Log Scale & $\log \sigma^{2}$ & $\theta_{\text {temp }}\left(25 v s 20^{\circ} \mathrm{C}\right)$ \\
\hline 1 & $2.00(1.65 ; 2.34)$ & $2.48(2.37 ; 2.60)$ & $0.35(0.03 ; 0.66)$ & $0.66(0.60 ; 0.73)$ \\
\hline 2 & $3.09(2.37 ; 3.82)$ & $2.16(2.04 ; 2.28)$ & $1.11(0.70 ; 1.52)$ & $0.79(0.72 ; 0.86)$ \\
\hline Meta-Analysis & $2.20(1.89 ; 2.51)$ & $2.32(2.24 ; 2.41)$ & $0.63(0.38 ; 0.88)$ & $0.72(0.68 ; 0.77)$ \\
\hline \multicolumn{5}{|l|}{ SIH treated } \\
\hline Replicate & Log Shape & Log Scale & $\log \sigma^{2}$ & $\theta_{\text {temp }}\left(25\right.$ vs $\left.20^{\circ} \mathrm{C}\right)$ \\
\hline 1 & $1.70(1.58 ; 1.81)$ & $2.93(2.89 ; 2.97)$ & $-3.93(-18.49 ; 10.63)$ & $0.76(0.72 ; 0.81)$ \\
\hline 2 & $1.87(1.64 ; 2.11)$ & $3.06(3.01 ; 3.12)$ & $-0.84(-1.90 ; 0.21)$ & $0.87(0.82 ; 0.93)$ \\
\hline Meta-Analysis & $1.73(1.63 ; 1.83)$ & $2.97(2.94 ; 3.00)$ & $-0.86(-1.91 ; 0.19)$ & $0.81(0.78 ; 0.84)$ \\
\hline
\end{tabular}




\section{Body length analysis}

Summary statistics and tests for normality of body length are included in Table S18. Not all length or derived data sets were normally distributed, as indicated below.

Table S18: Summary of body length comparisons between treatments and ages

\begin{tabular}{|c|c|c|c|c|c|c|c|c|c|c|}
\hline & Start & \begin{tabular}{|l} 
Day 1 \\
Control
\end{tabular} & $\begin{array}{l}\text { Day } 1 \\
\text { Lip-1 }\end{array}$ & $\begin{array}{l}\text { Day } 1 \\
\text { SIH }\end{array}$ & \begin{tabular}{|l|} 
Day 4 \\
Control
\end{tabular} & $\begin{array}{l}\text { Day } 4 \\
\text { Lip-1 }\end{array}$ & $\begin{array}{c}\text { Day } 4 \\
\text { SIH }\end{array}$ & \begin{tabular}{|c|} 
Day 8 \\
Control
\end{tabular} & $\begin{array}{l}\text { Day } 8 \\
\text { Lip-1 }\end{array}$ & $\begin{array}{l}\text { Day } 8 \\
\text { SIH }\end{array}$ \\
\hline Number of values & 125 & 82 & 98 & 90 & 71 & 49 & 68 & 17 & 10 & 21 \\
\hline Minimum & 876.2 & 1159 & 1114 & 1024 & 934.2 & 1023 & 1377 & 1229 & 1171 & 1532 \\
\hline $25 \%$ Percentile & 979.9 & 1341 & 1319 & 1371 & 1317 & 1277 & 1563 & 1346 & 1290 & 1651 \\
\hline Median & 1026 & 1387 & 1364 & 1412 & 1432 & 1345 & 1614 & 1403 & 1463 & 1704 \\
\hline $75 \%$ Percentile & 1084 & 1432 & 1403 & 1443 & 1519 & 1445 & 1648 & 1561 & 1524 & 1742 \\
\hline Maximum & 1271 & 1527 & 1488 & 1521 & 1660 & 1589 & 1773 & 1619 & 1554 & 1815 \\
\hline Mean & 1036 & 1387 & 1358 & 1400 & 1414 & 1344 & 1605 & 1440 & 1414 & 1696 \\
\hline Std. Deviation & 80.61 & 67.69 & 66.66 & 80.33 & 146.2 & 128.5 & 77.57 & 122.7 & 133.9 & 63.91 \\
\hline Std. Error of Mean & 7.21 & 7.475 & 6.734 & 8.467 & 17.35 & 18.36 & 9.406 & 29.76 & 42.33 & 13.95 \\
\hline Lower $95 \%$ CI of mean & 1022 & 1372 & 1345 & 1383 & 1379 & 1308 & 1586 & 1376 & 1318 & 1667 \\
\hline Upper $95 \%$ CI of mean & 1051 & 1402 & 1372 & 1417 & 1448 & 1381 & 1624 & 1503 & 1509 & 1725 \\
\hline \multicolumn{11}{|c|}{ D'Agostino \& Pearson normality test } \\
\hline $\mathrm{K} 2$ & 6.981 & 5.546 & 18.41 & 63.3 & 8.069 & 5.393 & 7.285 & 3.174 & 1.47 & 1.822 \\
\hline$p$ value & 0.0305 & 0.0625 & 0.0001 & $<0.0001$ & 0.0177 & 0.0675 & 0.0262 & 0.2045 & 0.4794 & 0.4021 \\
\hline $\begin{array}{l}\text { Passed normality test } \\
(\alpha=0.05) ?\end{array}$ & No & Yes & No & No & No & Yes & No & Yes & Yes & Yes \\
\hline$p$ value summary & $*$ & ns & $* * *$ & $* * * *$ & $*$ & ns & $*$ & ns & ns & $\mathrm{ns}$ \\
\hline
\end{tabular}


To compare between age and treatment groups a Kruskal-Wallace ANOVA was performed, followed by Dunn's multiple comparisons Post-hoc tests. There was a significant difference between body length $(\mathrm{H}(10)=432.6, p<0.0001)$ amongst the groups measured. The results of the pairwise comparisons, corrected for multiple comparisons, are shown in Table S19.

Table S19: Summary of body length comparisons between ages and treatments.

\begin{tabular}{|l|r|r|r|}
\hline Dunn's multiple comparisons test & Mean rank diff. & Significant? & Adjusted $p$ Value \\
\hline Start vs. Day 1 Control & -261.3 & Yes & $<0.0001$ \\
\hline Start vs. Day 1 Lip-1 & -219.1 & Yes & $<0.0001$ \\
\hline Start vs. Day 1 SIH & -305.1 & Yes & $<0.0001$ \\
\hline Day 1 Control vs. Day 1 Lip-1 & 42.24 & No & $>0.9999$ \\
\hline Day 1 Control vs. Day 1 SIH & -43.77 & No & $>0.9999$ \\
\hline Day 1 Control vs. Day 4 Control & -44.11 & No & $>0.9999$ \\
\hline Day 4 Control vs. Day 4 Lip-1 & 84.76 & No & 0.1743 \\
\hline Day 4 Control vs. Day 4 SIH & -184 & Yes & $<0.0001$ \\
\hline Day 4 Control vs. Day 8 Control & -7.775 & No & $>0.9999$ \\
\hline Day 8 Control vs. Day 8 Lip-1 & 9.976 & No & $>0.9999$ \\
\hline Day 8 Control vs. Day 8 SIH & -223.5 & Yes & 0.0023 \\
\hline Day 1 Lip-1 vs. Day 4 Lip-1 & -1.591 & No & $>0.9999$ \\
\hline Day 4 Lip-1 vs. Day 8 Lip-1 & -82.56 & No & $>0.9999$ \\
\hline Day 1 SIH vs. Day 4 SIH & -184.4 & Yes & $<0.0001$ \\
\hline Day 4 SIH vs. Day 8 SIH & -47.25 & No & $>0.9999$ \\
\hline
\end{tabular}




\section{Volume analysis}

Summary statistics and tests for normality of total worm volume are included in Table S20. Not all length or derived data sets were normally distributed, as indicated below.

Table S20: Summary of volume comparisons between treatments and ages

\begin{tabular}{|c|c|c|c|c|c|c|c|c|c|c|}
\hline & Start & $\begin{array}{c}\text { Day } 1 \\
\text { Control }\end{array}$ & Day 1 Lip-1 & Day $1 \mathrm{SIH}$ & $\begin{array}{l}\text { Day } 4 \\
\text { Control }\end{array}$ & Day 4 Lip-1 & Day $4 \mathrm{SIH}$ & $\begin{array}{l}\text { Day } 8 \\
\text { Control }\end{array}$ & Day 8 Lip-1 & Day $8 \mathrm{SIH}$ \\
\hline Number of values & 125 & 82 & 98 & 90 & 71 & 49 & 68 & 17 & 10 & 21 \\
\hline Minimum & 747.8 & 3115 & 2740 & 1731 & 3293 & 2258 & 4052 & 4939 & 4203 & 5461 \\
\hline $25 \%$ Percentile & 1589 & 4051 & 3883 & 4057 & 5049 & 4251 & 6677 & 5655 & 4882 & 7280 \\
\hline Median & 1867 & 4247 & 4293 & 4393 & 6103 & 5032 & 7183 & 6673 & 6147 & 7726 \\
\hline $75 \%$ Percentile & 2083 & 4576 & 4598 & 4740 & 6507 & 5648 & 7738 & 7465 & 6752 & 8407 \\
\hline Maximum & 3818 & 5249 & 5420 & 5800 & 8267 & 6712 & 8608 & 8696 & 7876 & 9210 \\
\hline Mean & 1901 & 4289 & 4209 & 4369 & 5810 & 4970 & 7111 & 6511 & 5958 & 7731 \\
\hline Std. Deviation & 495.1 & 417.5 & 540.8 & 643.1 & 1111 & 965.4 & 861.8 & 1043 & 1155 & 825.4 \\
\hline Std. Error of Mean & 44.28 & 46.1 & 54.63 & 67.79 & 131.8 & 137.9 & 104.5 & 252.9 & 365.2 & 180.1 \\
\hline Lower $95 \%$ CI of mean & 1813 & 4197 & 4101 & 4234 & 5547 & 4693 & 6903 & 5975 & 5132 & 7355 \\
\hline Upper $95 \%$ CI of mean & 1988 & 4380 & 4318 & 4504 & 6073 & 5248 & 7320 & 7047 & 6784 & 8106 \\
\hline \multicolumn{11}{|l|}{$\begin{array}{l}\text { D'Agostino \& Pearson normality } \\
\text { test }\end{array}$} \\
\hline $\mathrm{K} 2$ & 38.42 & 0.4213 & 2.657 & 37.57 & 2.331 & 1.45 & 12.99 & 0.3558 & 0.1231 & 4.164 \\
\hline$p$ value & $<0.0001$ & 0.8101 & 0.2649 & $<0.0001$ & 0.3118 & 0.4843 & 0.0015 & 0.8370 & 0.9403 & 0.1247 \\
\hline Passed normality test $(\alpha=0.05)$ ? & No & Yes & Yes & No & Yes & Yes & No & Yes & Yes & Yes \\
\hline$p$ value summary & $* * * *$ & $\mathrm{~ns}$ & $\mathrm{~ns}$ & $* * * *$ & $\mathrm{~ns}$ & $\mathrm{~ns}$ & $* *$ & $\mathrm{~ns}$ & $\mathrm{~ns}$ & $\mathrm{~ns}$ \\
\hline
\end{tabular}


There was a significant difference between body volume $(\mathrm{H}(10)=489, p<0.0001)$ amongst the groups measured. Comparisons between age and treatment groups a Kruskal-Wallace ANOVA was performed, followed by Dunn's multiple comparisons Post-hoc tests. The results of the pairwise comparisons, corrected for multiple comparisons, are shown in Table S21.

Table S21: Summary of volume comparisons between ages and treatments.

\begin{tabular}{|l|r|r|r|}
\hline Dunn's multiple comparisons test & Mean rank diff. & Significant? & Adjusted $p$ Value \\
\hline \hline Start vs. Day 1 Control & -214.9 & Yes & $<0.0001$ \\
\hline Start vs. Day 1 Lip-1 & -205.6 & Yes & $<0.0001$ \\
\hline Start vs. Day 1 SIH & -236.4 & Yes & $<0.0001$ \\
\hline Day 1 Control vs. Day 1 Lip-1 & 9.301 & No & $>0.9999$ \\
\hline Day 1 Control vs. Day 1 SIH & -21.45 & No & $>0.9999$ \\
\hline Day 1 Control vs. Day 4 Control & -175 & Yes & $<0.0001$ \\
\hline Day 4 Control vs. Day 4 Lip-1 & 80.42 & No & 0.2630 \\
\hline Day 4 Control vs. Day 4 SIH & -102.6 & Yes & 0.0136 \\
\hline Day 4 Control vs. Day 8 Control & -66.73 & No & $>0.9999$ \\
\hline Day 8 Control vs. Day 8 Lip-1 & 54.78 & No & $>0.9999$ \\
\hline Day 8 Control vs. Day 8 SIH & -70.02 & No & $>0.9999$ \\
\hline Day 1 Lip-1 vs. Day 4 Lip-1 & -103.9 & Yes & 0.0169 \\
\hline Day 4 Lip-1 vs. Day 8 Lip-1 & -92.37 & No & $>0.9999$ \\
\hline Day 1 SIH vs. Day 4 SIH & -256.2 & Yes & $<0.0001$ \\
\hline Day 4 SIH vs. Day 8 SIH & -34.11 & No & $>0.9999$ \\
\hline
\end{tabular}

\section{Movement analysis}

Movement parameters measured included maximum velocity (shown in Figure 6e), mean velocity and total distance travelled. The latter two datasets are represented in Figures S12 and S13.

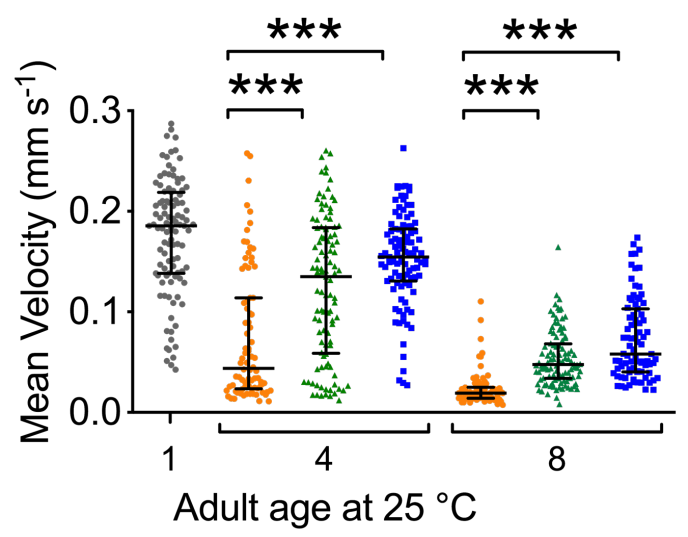

Figure S12 Estimates of mean velocity $\left(\mathrm{mm} \mathrm{s}^{-1}\right)$ achieved by aged and treated cohorts of C. elegans. (Grey=starting population, red $=$ control, green $=$ Lip-1 treated, blue $=$ SIH-treated) Treatment with either Lip-1 or SIH attenuates the age-related decline in mean velocity (Kruskal-Wallis ANOVA: H(7)= 339.2, $p<0.0001$ ), see Supplemental Table S24 for summary data and Table S25 for pair-wise comparisons). 


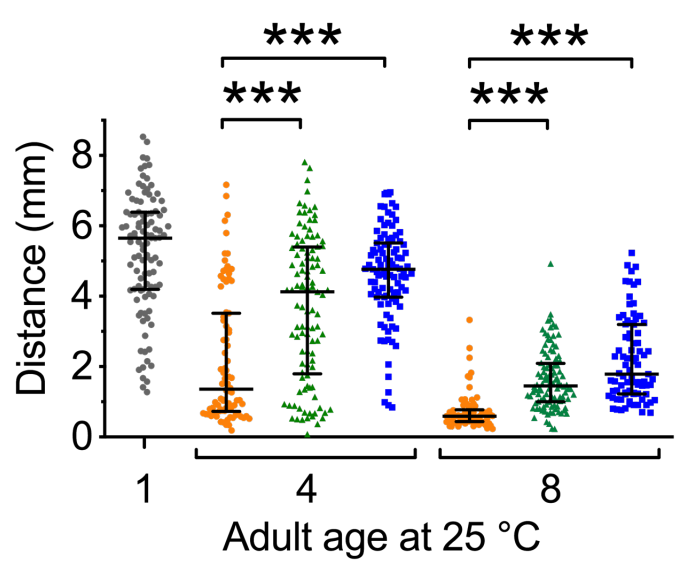

Figure S13 Estimates of total distance travelled (in $30 \mathrm{~s}$ ) by aged and treated cohorts of C. elegans. (Grey=starting population, red=control, green=Lip-1 treated, blue=SIH-treated) Treatment with either Lip-1 or SIH attenuates the age-related decline in distance travelled (Kruskal-Wallis ANOVA: H(7)= 340.6, $p<0.0001$ )see Supplemental Table S26 for summary data and Table S27 for pair-wise comparisons).

Summary statistics and tests for normality of maximum velocity $\left(\mathrm{mm} \mathrm{s}^{-1}\right)$ are included in Table S22. Not all data sets were normally distributed, as indicated below.

Table S22: Summary of maximum velocity results across treatments and ages

\begin{tabular}{|c|c|c|c|c|c|c|c|}
\hline & $\begin{array}{l}\text { Day } 1 \\
\text { Control }\end{array}$ & $\begin{array}{c}\text { Day } 4 \\
\text { Control }\end{array}$ & $\begin{array}{c}\text { Day } 8 \\
\text { Control }\end{array}$ & Day 4 SIH & Day $8 \mathrm{SIH}$ & \begin{tabular}{|c|} 
Day 4 Lip- \\
1
\end{tabular} & $\begin{array}{c}\text { Day } 8 \text { Lip- } \\
1\end{array}$ \\
\hline Number of values & 103 & 82 & 73 & 99 & 83 & 105 & 104 \\
\hline Minimum & 0.1832 & 0.04309 & 0.03738 & 0.105 & 0.1028 & 0.06351 & 0.05622 \\
\hline $25 \%$ Percentile & 0.3731 & 0.1294 & 0.06372 & 0.3442 & 0.174 & 0.2711 & 0.1478 \\
\hline Median & 0.4509 & 0.2304 & 0.09115 & 0.3733 & 0.2513 & 0.3762 & 0.1922 \\
\hline $75 \%$ Percentile & 0.5491 & 0.3364 & 0.1254 & 0.4755 & 0.3506 & 0.4861 & 0.2452 \\
\hline Maximum & 0.8264 & 0.6134 & 0.3802 & 0.7127 & 0.6458 & 0.7204 & 0.5563 \\
\hline Mean & 0.4692 & 0.2536 & 0.109 & 0.392 & 0.2847 & 0.37 & 0.2084 \\
\hline Std. Deviation & 0.1457 & 0.1496 & 0.06568 & 0.1087 & 0.1356 & 0.1588 & 0.09062 \\
\hline Std. Error of Mean & 0.01435 & 0.01653 & 0.007687 & 0.01093 & 0.01489 & 0.01549 & 0.008886 \\
\hline $\begin{array}{l}\text { Lower 95\% CI of } \\
\text { mean }\end{array}$ & 0.4408 & 0.2207 & 0.09367 & 0.3704 & 0.2551 & 0.3393 & 0.1908 \\
\hline $\begin{array}{l}\text { Upper 95\% CI of } \\
\text { mean }\end{array}$ & 0.4977 & 0.2865 & 0.1243 & 0.4137 & 0.3143 & 0.4007 & 0.226 \\
\hline \multicolumn{8}{|c|}{ D'Agostino \& Pearson normality test } \\
\hline K2 & 4.785 & 8.157 & 44.36 & 0.9424 & 10.76 & 3.145 & 23.3 \\
\hline$p$ value & 0.0914 & 0.0169 & $<0.0001$ & 0.6242 & 0.0046 & 0.2075 & $<0.0001$ \\
\hline $\begin{array}{l}\text { Passed normality test } \\
(\alpha=0.05) ?\end{array}$ & Yes & No & No & Yes & No & Yes & No \\
\hline$p$ value summary & ns & $*$ & $* * * *$ & ns & $* *$ & ns & $* * * *$ \\
\hline
\end{tabular}


To compare between age and treatment groups a Kruskal-Wallace ANOVA was performed, followed by Dunn's multiple comparisons post-hoc tests. There was a significant difference between maximum velocity $(H(7)=298.5, p<0.0001)$ amongst the groups measured. The results of the pairwise comparisons, corrected for multiple comparisons, are shown in Table S23.

Table S23: Summary of maximum velocity comparisons between ages and treatments.

\begin{tabular}{|l|r|c|r|}
\hline Dunn's multiple comparisons test & Mean rank diff. & Significant? & Adjusted $p$ Value \\
\hline \hline Day 1 Control vs. Day 4 Control & 230.5 & Yes & $<0.0001$ \\
\hline Day 1 Control vs. Day 8 Control & 411.2 & Yes & $<0.0001$ \\
\hline Day 4 Control vs. Day 8 Control & 180.7 & Yes & $<0.0001$ \\
\hline Day 4 Control vs. Day 4 SIH & -168.4 & Yes & $<0.0001$ \\
\hline Day 4 Control vs. Day 4 Lip-1 & -133.6 & Yes & $<0.0001$ \\
\hline Day 8 Control vs. Day 8 SIH & -220.9 & Yes & $<0.0001$ \\
\hline Day 8 Control vs. Day 8 Lip-1 & -132.7 & Yes & $<0.0001$ \\
\hline Day 4 SIH vs. Day 4 Lip-1 & 34.84 & No & $>0.9999$ \\
\hline Day 8 SIH vs. Day 8 Lip-1 & 88.29 & Yes & 0.0110 \\
\hline
\end{tabular}

\section{Mean velocity}

Summary statistics and tests for normality of mean velocity $\left(\mathrm{mm} \mathrm{s}^{-1}\right)$ are included in Table S24. Not all data sets were normally distributed, as indicated below.

Table S24: Summary of mean velocity results across treatments and ages

\begin{tabular}{|c|c|c|c|c|c|c|c|}
\hline & $\begin{array}{l}\text { Day 1 } \\
\text { Control } \\
\end{array}$ & $\begin{array}{c}\text { Day } 4 \\
\text { Control }\end{array}$ & $\begin{array}{l}\text { Day } 8 \\
\text { Control }\end{array}$ & Day 4 SIH & Day $8 \mathrm{SIH}$ & \begin{tabular}{|c|} 
Day 4 Lip- \\
1
\end{tabular} & $\begin{array}{c}\text { Day } 8 \text { Lip- } \\
1\end{array}$ \\
\hline Number of values & 103 & 82 & 73 & 99 & 83 & 105 & 104 \\
\hline Minimum & 0.04246 & 0.01112 & 0.007567 & 0.02713 & 0.02239 & 0.01219 & 0.008319 \\
\hline $25 \%$ Percentile & 0.1382 & 0.02355 & 0.01407 & 0.1307 & 0.04028 & 0.05892 & 0.03386 \\
\hline Median & 0.1856 & 0.04389 & 0.01907 & 0.1545 & 0.05813 & 0.1349 & 0.04763 \\
\hline $75 \%$ Percentile & 0.2189 & 0.1139 & 0.02507 & 0.1824 & 0.1029 & 0.1839 & 0.06818 \\
\hline Maximum & 0.2873 & 0.2578 & 0.1104 & 0.2629 & 0.1739 & 0.2606 & 0.1644 \\
\hline Mean & 0.1756 & 0.07449 & 0.02376 & 0.1528 & 0.07262 & 0.1244 & 0.05407 \\
\hline Std. Deviation & 0.05753 & 0.06507 & 0.01791 & 0.0464 & 0.04028 & 0.07094 & 0.02726 \\
\hline Std. Error of Mean & 0.005669 & 0.007186 & 0.002096 & 0.004663 & 0.004421 & 0.006923 & 0.002673 \\
\hline $\begin{array}{l}\text { Lower } 95 \% \text { CI of } \\
\text { mean }\end{array}$ & 0.1644 & 0.06019 & 0.01958 & 0.1435 & 0.06382 & 0.1106 & 0.04877 \\
\hline $\begin{array}{l}\text { Upper 95\% CI of } \\
\text { mean }\end{array}$ & 0.1868 & 0.08878 & 0.02793 & 0.162 & 0.08141 & 0.1381 & 0.05937 \\
\hline \multicolumn{8}{|c|}{ D'Agostino \& Pearson normality test } \\
\hline $\mathrm{K} 2$ & 3.86 & 13.86 & 66.08 & 6.268 & 8.786 & 29.36 & 21.66 \\
\hline$p$ value & 0.1451 & 0.0010 & $<0.0001$ & 0.0435 & 0.0124 & $<0.0001$ & $<0.0001$ \\
\hline $\begin{array}{l}\text { Passed normality test } \\
(\alpha=0.05) ?\end{array}$ & Yes & No & No & No & No & No & No \\
\hline$p$ value summary & ns & $* * *$ & $* * * *$ & $*$ & $*$ & $* * * *$ & $* * * *$ \\
\hline
\end{tabular}


To compare between age and treatment groups a Kruskal-Wallace ANOVA was performed, followed by Dunn's multiple comparisons Post-hoc tests. There was a significant difference between mean velocity $(\mathrm{H}(7)=339.2, p<0.0001)$ amongst the groups measured. The results of the pairwise comparisons, corrected for multiple comparisons, are shown in Table S25.

Table S25: Summary of mean velocity comparisons between ages and treatments.

\begin{tabular}{|l|r|c|r|}
\hline Dunn's multiple comparisons test & Mean rank diff. & Significant? & Adjusted $p$ Value \\
\hline \hline Day 1 Control vs. Day 4 Control & 259.2 & Yes & $<0.0001$ \\
\hline Day 1 Control vs. Day 8 Control & 427.8 & Yes & $<0.0001$ \\
\hline Day 4 Control vs. Day 8 Control & 168.6 & Yes & $<0.0001$ \\
\hline Day 4 Control vs. Day 4 SIH & -215.1 & Yes & $<0.0001$ \\
\hline Day 4 Control vs. Day 4 Lip-1 & -135.7 & Yes & $<0.0001$ \\
\hline Day 8 Control vs. Day 8 SIH & -192.7 & Yes & $<0.0001$ \\
\hline Day 8 Control vs. Day 8 Lip-1 & -141.2 & Yes & $<0.0001$ \\
\hline Day 4 SIH vs. Day 4 Lip-1 & 79.45 & Yes & 0.0224 \\
\hline Day 8 SIH vs. Day 8 Lip-1 & 51.53 & No & 0.5569 \\
\hline
\end{tabular}

Total distance travelled

Summary statistics and tests for normality of total distance travelled ( $\mathrm{mm})$ are included in Table S26. Not all data were normally distributed, as indicated below.

Table S26: Summary of distance travelled results across treatments and ages

\begin{tabular}{|c|c|c|c|c|c|c|c|}
\hline & $\begin{array}{c}\text { Day } 1 \\
\text { Control } \\
\end{array}$ & $\begin{array}{l}\text { Day } 4 \\
\text { Control } \\
\end{array}$ & $\begin{array}{c}\text { Day } 8 \\
\text { Control } \\
\end{array}$ & $\begin{array}{c}\text { Day } 4 \\
\text { SIH }\end{array}$ & $\begin{array}{c}\text { Day } 8 \\
\text { SIH }\end{array}$ & $\begin{array}{l}\text { Day } 4 \\
\text { Lip-1 }\end{array}$ & $\begin{array}{r}\text { Day } 8 \\
\text { Lip-1 } \\
\end{array}$ \\
\hline Number of values & 103 & 82 & 73 & 99 & 83 & 105 & 104 \\
\hline Minimum & 1.28 & 0.1883 & 0.2346 & 0.8402 & 0.6912 & 0.07633 & 0.2338 \\
\hline $25 \%$ Percentile & 4.197 & 0.7269 & 0.4349 & 3.977 & 1.224 & 1.797 & 1.001 \\
\hline Median & 5.649 & 1.357 & 0.5888 & 4.766 & 1.784 & 4.126 & 1.451 \\
\hline $75 \%$ Percentile & 6.388 & 3.521 & 0.7714 & 5.513 & 3.193 & 5.397 & 2.092 \\
\hline Maximum & 8.534 & 7.169 & 3.324 & 6.951 & 5.235 & 7.817 & 4.926 \\
\hline Mean & 5.24 & 2.216 & 0.7247 & 4.619 & 2.192 & 3.685 & 1.627 \\
\hline Std. Deviation & 1.68 & 1.865 & 0.5329 & 1.349 & 1.192 & 2.064 & 0.849 \\
\hline Std. Error of Mean & 0.1656 & 0.2059 & 0.06237 & 0.1356 & 0.1308 & 0.2014 & 0.08325 \\
\hline Lower $95 \%$ CI of mean & 4.911 & 1.806 & 0.6003 & 4.35 & 1.932 & 3.285 & 1.462 \\
\hline Upper $95 \%$ CI of mean & 5.568 & 2.626 & 0.849 & 4.888 & 2.452 & 4.084 & 1.792 \\
\hline \multicolumn{8}{|c|}{ D'Agostino \& Pearson normality test } \\
\hline K2 & 4.494 & 10.94 & 63.28 & 9.719 & 8.451 & 27.91 & 16.99 \\
\hline$p$ value & 0.1057 & 0.0042 & $<0.0001$ & 0.0078 & 0.0146 & $<0.0001$ & 0.0002 \\
\hline $\begin{array}{l}\text { Passed normality test } \\
(\alpha=0.05) ?\end{array}$ & Yes & No & No & No & No & No & No \\
\hline$p$ value summary & ns & $* *$ & $* * * *$ & $* *$ & $*$ & $* * * *$ & $* * *$ \\
\hline
\end{tabular}


To compare between age and treatment groups a Kruskal-Wallace ANOVA was performed, followed by Dunn's multiple comparisons Post-hoc tests. There was a significant difference between total distance travelled $(\mathrm{H}(7)=340.6, p<0.0001)$ amongst the groups measured. The results of the pairwise comparisons, corrected for multiple comparisons, are shown in Table S27.

Table S27: Summary of total distance travelled comparisons between ages and treatments.

\begin{tabular}{|l|r|r|r|}
\hline Dunn's multiple comparisons test & Mean rank diff. & Significant? & Adjusted $p$ value \\
\hline Day 1 Control vs. Day 4 Control & 260.6 & Yes & $<0.0001$ \\
\hline Day 1 Control vs. Day 8 Control & 425.7 & Yes & $<0.0001$ \\
\hline Day 4 Control vs. Day 8 Control & 165.1 & Yes & $<0.0001$ \\
\hline Day 4 Control vs. Day 4 SIH & -218.3 & Yes & $<0.0001$ \\
\hline Day 4 Control vs. Day 4 Lip-1 & -133.1 & Yes & $<0.0001$ \\
\hline Day 8 Control vs. Day 8 SIH & -189.4 & Yes & $<0.0001$ \\
\hline Day 8 Control vs. Day 8 Lip-1 & -134.8 & Yes & $<0.0001$ \\
\hline Day 4 SIH vs. Day 4 Lip-1 & 85.29 & Yes & 0.0105 \\
\hline Day 8 SIH vs. Day 8 Lip-1 & 54.61 & No & 0.4305 \\
\hline
\end{tabular}

\section{Correlation of estimated movement parameters}

Pooling all groups and ages reveals that all movement parameters (maximum velocity, mean velocity and distance travelled in 30s) are all positively correlated, as shown in Figure S14.

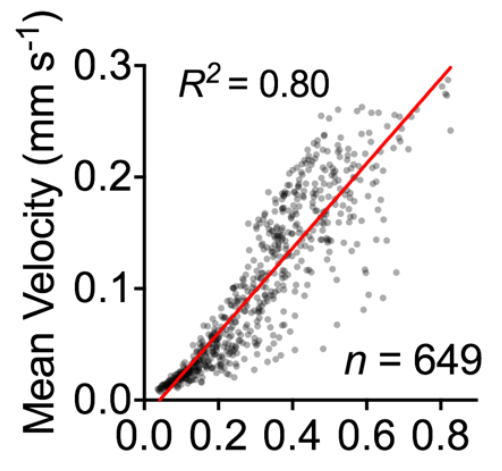

Max. Velocity $\left(\mathrm{mm} \mathrm{s}^{-1}\right)$

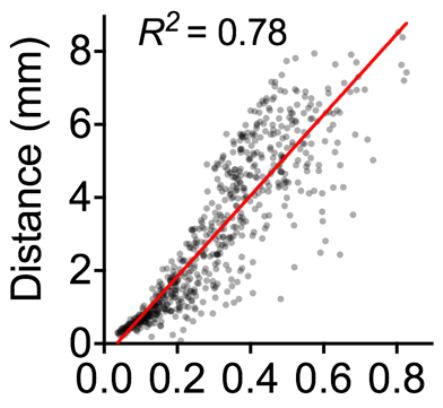

Max. Velocity $\left(\mathrm{mm} \mathrm{s}^{-1}\right)$

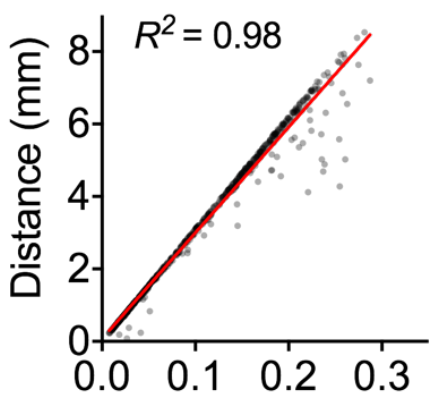

Mean Velocity $\left(\mathrm{mm} \mathrm{s}^{-1}\right)$

Figure S14 Correlation of estimated movement parameters. 


\section{Antibiotic tests}

To determine whether the increased lifespan seen with SIH treatment could be explained solely by an antibiotic effect of iron reduction, nematodes were treated with ampicillin, with and without SIH coadministration. Even in the presence of ampicillin, SIH increased median lifespan by 6 days, similar to its benefits in the absence of ampicillin (median increase of 7 days), as shown in Figure S15.

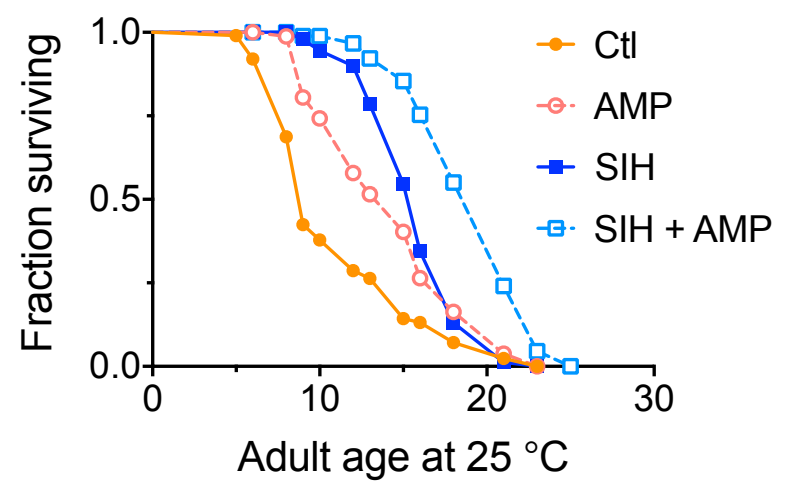

Figure S15: Administration of the antibiotic ampicillin and $\mathrm{SIH}$

$\mathrm{AMP}=5 \mu \mathrm{g} / \mathrm{ml}$ ampicillin. Median survival - Log-rank (Mantel-Cox) test: Control $(\mathrm{Ctl})=9$ days; $\mathrm{AMP}=15$ days $(p<0.0001) ; 250 \mu \mathrm{M} \mathrm{SIH}=16$ days $(p<0.0001) ; 250 \mu \mathrm{M} \mathrm{SIH}+\mathrm{AMP}=21$ days $(p<0.0001)$. 


\section{References}

1 Dixon, S. J. et al. Ferroptosis: An iron-dependent form of nonapoptotic cell death. Cell 149, 1060-1072, doi:10.1016/j.cell.2012.03.042 (2012).

2 James, S. A. et al. Direct in vivo imaging of ferrous iron dyshomeostasis in ageing Caenorhabditis elegans. Chemical Science 6, 2952-2962, doi:10.1039/C5SC00233H (2015).

3 Massie, H. R., Aiello, V. R. \& Banziger, V. Iron accumulation and lipid peroxidation in aging C57BL/6J mice. Experimental gerontology 18, 277-285 (1983).

4 Massie, H. R., Aiello, V. R. \& Williams, T. R. Iron accumulation during development and ageing of Drosophila. Mech Ageing Dev 29, 215-220 (1985).

5 Ward, R. J., Zucca, F. A., Duyn, J. H. \& Crichton, R. R. The role of iron in brain ageing and neurodegenerative disorders. Lancet Neurology 13, 1045-1060 (2014).

6 Hare, D., Ayton, S., Bush, A. \& Lei, P. A delicate balance: Iron metabolism and diseases of the brain. Frontiers in Aging Neuroscience 5, doi:10.3389/fnagi.2013.00034 (2013).

7 Hare, D. J. et al. Is early-life iron exposure critical in neurodegeneration? Nature Reviews Neurology 11, 536-544 (2015).

8 Stockwell, B. R. et al. Ferroptosis: A Regulated Cell Death Nexus Linking Metabolism, Redox Biology, and Disease. Cell 171, 273-285, doi:10.1016/j.cell.2017.09.021 (2017).

9 Viswanathan, V. S. et al. Dependency of a therapy-resistant state of cancer cells on a lipid peroxidase pathway. Nature 547, 453-457, doi:10.1038/nature23007 (2017).

10 Yang, W. S. \& Stockwell, B. R. Ferroptosis: Death by Lipid Peroxidation. Trends Cell Biol 26, 165-176, doi:10.1016/j.tcb.2015.10.014 (2016).

11 Tuo, Q. z. et al. Tau-mediated iron export prevents ferroptotic damage after ischemic stroke. Molecular Psychiatry 22, 1520, doi:10.1038/mp.2017.171

https://www.nature.com/articles/mp2017171\#supplementary-information (2017).

12 Jiang, L. et al. Ferroptosis as a p53-mediated activity during tumour suppression. Nature 520, 57-62, doi:10.1038/nature14344 (2015).

13 Conrad, M., Angeli, J. P., Vandenabeele, P. \& Stockwell, B. R. Regulated necrosis: disease relevance and therapeutic opportunities. Nat Rev Drug Discov, doi:10.1038/nrd.2015.6 (2016).

14 Doll, S. et al. ACSL4 dictates ferroptosis sensitivity by shaping cellular lipid composition. Nat Chem Biol 13, 91-98, doi:10.1038/nchembio.2239 (2017).

15 Doll, S. \& Conrad, M. Iron and ferroptosis: A still ill-defined liaison. IUBMB Life 69, 423-434, doi:10.1002/iub.1616 (2017).

16 James, S. A. et al. $\varphi$ XANES: In vivo imaging of metal-protein coordination environments. Scientific Reports 6, 20350, doi:10.1038/srep20350 (2016).

$17 \mathrm{Xu}$, J. et al. The emerging role of iron dyshomeostasis in the mitochondrial decay of aging. Mechanisms of Ageing and Development 131, 487-493, doi:10.1016/j.mad.2010.04.007 (2010).

18 Gourley, B. L., Parker, S. B., Jones, B. J., Zumbrennen, K. B. \& Leibold, E. A. Cytosolic Aconitase and Ferritin Are Regulated by Iron inCaenorhabditis elegans. Journal of Biological Chemistry 278, 3227-3234, doi:10.1074/jbc.M210333200 (2003).

19 Hu, Y.-O., Wang, Y., Ye, B.-P. \& Wang, D.-Y. Phenotypic and Behavioral Defects Induced by Iron Exposure Can Be Transferred to Progeny in Caenorhabditis elegans. Biomedical and Environmental Sciences 21, 467-473, doi:10.1016/s0895-3988(09)60004-0 (2008).

20 Valentini, S. et al. Manipulation of in vivo iron levels can alter resistance to oxidative stress without affecting ageing in the nematode C. elegans. Mech Ageing Dev 133, 282-290, doi:10.1016/j.mad.2012.03.003 (2012).

21 Klang, I. M. et al. Iron promotes protein insolubility and aging in C. elegans. Aging 6, 975-991 (2014).

22 Hider, R. C. \& Kong, X. L. Glutathione: a key component of the cytoplasmic labile iron pool. Biometals 24, 1179-1187, doi:10.1007/s10534-011-9476-8 (2011).

23 Zilka, O. et al. On the Mechanism of Cytoprotection by Ferrostatin-1 and Liproxstatin-1 and the Role of Lipid Peroxidation in Ferroptotic Cell Death. ACS Cent Sci 3, 232-243, doi:10.1021/acscentsci.7b00028 (2017). 
24 Kalinowski, D. S. \& Richardson, D. R. The evolution of iron chelators for the treatment of iron overload disease and cancer. Pharmacol Rev 57, 547-583, doi:10.1124/pr.57.4.2 (2005).

25 Dixon, S. J. \& Stockwell, B. R. The role of iron and reactive oxygen species in cell death. Nat Chem Biol 10, 9-17, doi:10.1038/nchembio.1416 (2014).

26 Yang, W. S. et al. Regulation of ferroptotic cancer cell death by GPX4. Cell 156, 317-331, doi:10.1016/j.cell.2013.12.010 (2014).

27 Friedmann Angeli, J. P. et al. Inactivation of the ferroptosis regulator Gpx4 triggers acute renal failure in mice. Nat Cell Biol 16, 1180-1191, doi:10.1038/ncb3064 (2014).

28 Sakamoto, T., Maebayashi, K., Nakagawa, Y. \& Imai, H. Deletion of the four phospholipid hydroperoxide glutathione peroxidase genes accelerates aging in Caenorhabditis elegans. Genes Cells 19, 778-792, doi:10.1111/gtc.12175 (2014).

29 Urban, N. et al. Non-linear impact of glutathione depletion on C. elegans life span and stress resistance. Redox Biology 11, 502-515, doi:10.1016/j.redox.2016.12.003 (2017).

30 Valmas, N. \& Ebert, P. R. Comparative toxicity of fumigants and a phosphine synergist using a novel containment chamber for the safe generation of concentrated phosphine gas. PLoS One 1, e130, doi:10.1371/journal.pone.0000130 (2006).

31 Chen, Y. L., Kong, X., Xie, Y. \& Hider, R. C. The interaction of pyridoxal isonicotinoyl hydrazone (PIH) and salicylaldehyde isonicotinoyl hydrazone (SIH) with iron. J Inorg Biochem 180, 194-203, doi:10.1016/j.jinorgbio.2017.12.007 (2018).

32 Shah, R., Shchepinov, M. S. \& Pratt, D. A. Resolving the Role of Lipoxygenases in the Initiation and Execution of Ferroptosis. ACS Central Science 4, 387-396, doi:10.1021/acscentsci.7b00589 (2018).

33 Linkermann, A. et al. Synchronized renal tubular cell death involves ferroptosis. Proc Natl Acad Sci U S A 111, 16836-16841, doi:10.1073/pnas.1415518111 (2014).

34 Feng, H. \& Stockwell, B. R. Unsolved mysteries: How does lipid peroxidation cause ferroptosis? PLoS Biol 16, e2006203, doi:10.1371/journal.pbio.2006203 (2018).

35 Ganio, K., James, S. A., Hare, D. J., Roberts, B. R. \& McColl, G. Accurate biometal quantification per individual Caenorhabditis elegans. The Analyst 141, 1434-1439, doi:10.1039/C5AN02544C (2016).

36 Westre, T. E. et al. A Multiplet Analysis of Fe K-Edge 1s $\rightarrow$ 3d Pre-Edge Features of Iron Complexes. Journal of the American Chemical Society 119, 6297-6314, doi:10.1021/ja964352a (1997).

37 Stroustrup, N. et al. The temporal scaling of Caenorhabditis elegans ageing. Nature 530, 103107, doi:10.1038/nature16550 (2016).

38 Piper, Matthew D. W., Partridge, L., Raubenheimer, D. \& Simpson, Stephen J. Dietary Restriction and Aging: A Unifying Perspective. Cell metabolism 14, 154-160, doi:https://doi.org/10.1016/j.cmet.2011.06.013 (2011).

39 Jenkins, N. L., McColl, G. \& Lithgow, G. J. Fitness cost of extended lifespan in Caenorhabditis elegans. Proceedings of the Royal Society of London B: Biological Sciences 271, 2523-2526, doi:10.1098/rspb.2004.2897 (2004).

40 Walker, D. W., McColl, G., Jenkins, N. L., Harris, J. \& Lithgow, G. J. Evolution of lifespan in C. elegans. Nature 405, 296-297, doi:10.1038/35012693 (2000).

41 Hahm, J.-H. et al. C. elegans maximum velocity correlates with healthspan and is maintained in worms with an insulin receptor mutation. Nature Communications 6, 8919, doi:10.1038/ncomms9919 (2015).

42 Gaschler, M. M. et al. FINO2 initiates ferroptosis through GPX4 inactivation and iron oxidation. Nat Chem Biol 14, 507-515, doi:10.1038/s41589-018-0031-6 (2018).

43 Gems, D. \& Partridge, L. Genetics of longevity in model organisms: debates and paradigm shifts. Annu Rev Physiol 75, 621-644, doi:10.1146/annurev-physiol-030212-183712 (2013).

44 Muckenthaler, M. U., Rivella, S., Hentze, M. W. \& Galy, B. A Red Carpet for Iron Metabolism. Cell 168, 344-361, doi:https://doi.org/10.1016/j.cell.2016.12.034 (2017).

45 Coffey, R. \& Ganz, T. Iron homeostasis: An anthropocentric perspective. The Journal of biological chemistry 292, 12727-12734, doi:10.1074/jbc.R117.781823 (2017). 
46 Newcombe, R. G. Two-sided confidence intervals for the single proportion: comparison of seven methods. Stat Med 17, 857-872 (1998).

47 Brenner, S. The genetics of Caenorhabditis elegans. Genetics 77, 71-94 (1974).

48 Rahman, I., Kode, A. \& Biswas, S. K. Assay for quantitative determination of glutathione and glutathione disulfide levels using enzymatic recycling method. Nat Protoc 1, 3159-3165, doi:10.1038/nprot.2006.378 (2006).

49 Caito, S. W. \& Aschner, M. Quantification of Glutathione in Caenorhabditis elegans. Curr Protoc Toxicol 64, 618 11-16, doi:10.1002/0471140856.tx0618s64 (2015).

50 Hare, D. J., Roberts, B. R. \& McColl, G. Profiling changes to natively-bound metals during Caenorhabditis elegans development. $R S C A d v$ 6, 113689-113693, doi:10.1039/C6RA22084C (2016).

51 James, S. A. et al. Direct in vivo imaging of essential bioinorganics in Caenorhabditis elegans. Metallomics 5, 627-635, doi:10.1039/c3mt00010a (2013).

52 Hare, D. J. et al. High-resolution complementary chemical imaging of bio-elements in Caenorhabditis elegans. Metallomics 8, 156-160, doi:10.1039/C5MT00288E (2016).

53 Paterson, D. et al. The X-ray Fluorescence Microscopy Beamline at the Australian Synchrotron. Aip Conf Proc 1365, 219-222 (2011).

54 Kirkham, R. et al. The Maia Spectroscopy Detector System: Engineering for Integrated Pulse Capture, Low - Latency Scanning and Real - Time Processing. AIP Conference Proceedings 1234, 240-243, doi:10.1063/1.3463181 (2010).

55 Ryan, C. G. Quantitative trace element imaging using PIXE and the nuclear microprobe. Int $J$ Imag Syst Tech 11, 219-230, doi:DOI 10.1002/ima.1007 (2000).

56 Davies, K. M. et al. Comparative Study of Metal Quantification in Neurological Tissue Using Laser Ablation-Inductively Coupled Plasma-Mass Spectrometry Imaging and X-ray Fluorescence Microscopy. Anal Chem 87, 6639-6645, doi:10.1021/acs.analchem.5b01454 (2015).

57 Schneider, C. A., Rasband, W. S. \& Eliceiri, K. W. NIH Image to ImageJ: 25 years of image analysis. Nat Meth 9, 671-675 (2012).

58 Kadachi, A. N. \& Al-Eshaikh, M. A. Limits of detection in XRF spectroscopy. X-Ray Spectrom 41, 350-354, doi:10.1002/xrs.2412 (2012).

59 Berry, A. J., O’Neill, H. S. C., Jayasuriya, K. D., Campbell, S. J. \& Foran, G. J. XANES calibrations for the oxidation state of iron in a silicate glass. American Mineralogist 88, $967-$ 977 (2003).

60 McColl, G. et al. Insulin-like signaling determines survival during stress via posttranscriptional mechanisms in C. elegans. Cell metabolism 12, 260-272, doi:10.1016/j.cmet.2010.08.004 (2010).

61 Garigan, D. et al. Genetic analysis of tissue aging in Caenorhabditis elegans: a role for heatshock factor and bacterial proliferation. Genetics 161, 1101-1112 (2002).

62 Bauer, A. W., Kirby, W. M., Sherris, J. C. \& Turck, M. Antibiotic susceptibility testing by a standardized single disk method. Am J Clin Pathol 45, 493-496 (1966).

63 Lucanic, M. et al. Impact of genetic background and experimental reproducibility on identifying chemical compounds with robust longevity effects. Nat Commun 8, 14256, doi:10.1038/ncomms14256 (2017).

64 Petrascheck, M. \& Miller, D. L. Computational Analysis of Lifespan Experiment Reproducibility. Front Genet 8, 92, doi:10.3389/fgene.2017.00092 (2017).

65 Moore, B. T., Jordan, J. M. \& Baugh, L. R. WormSizer: high-throughput analysis of nematode size and shape. PLoS One 8, e57142, doi:10.1371/journal.pone.0057142 (2013).

66 Nussbaum-Krammer, C. I., Neto, M. F., Brielmann, R. M., Pedersen, J. S. \& Morimoto, R. I. Investigating the spreading and toxicity of prion-like proteins using the metazoan model organism C. elegans. J Vis Exp, 52321, doi:10.3791/52321 (2015).

67 Schindelin, J. et al. Fiji: an open-source platform for biological-image analysis. Nat Methods 9, 676-682, doi:10.1038/nmeth.2019 (2012).

68 Wilson, E. B. Probable inference, the law of succession, and statistical inference. J Am Stat Assoc 22, 209-212, doi:Doi 10.2307/2276774 (1927). 
69 Campbell, I. Chi-squared and Fisher-Irwin tests of two-by-two tables with small sample recommendations. Stat Med 26, 3661-3675, doi:10.1002/sim.2832 (2007).

70 Richardson, J. T. The analysis of 2 x 2 contingency tables--yet again. Stat Med 30, 890; author reply 891-892, doi:10.1002/sim.4116 (2011).

71 Fleming, T. R., O'Fallon, J. R., O'Brien, P. C. \& Harrington, D. P. Modified KolmogorovSmirnov Test Procedures with Application to Arbitrarily Right-Censored Data. Biometrics 36, 607-625, doi:10.2307/2556114 (1980).

72 Buckley, J. \& James, I. A. N. Linear regression with censored data. Biometrika 66, 429-436, doi:10.1093/biomet/66.3.429 (1979).

73 Lindsey, J. C. \& Ryan, L. M. Methods for interval-censored data. Statistics in Medicine 17, 219-238, doi:10.1002/(SICI)1097-0258(19980130)17:2<219::AID-SIM735>3.0.CO;2-O (1998). 